\title{
A Parametric Study of Inertial Particle Separator Geometry
}

\author{
A Thesis Presented to \\ The Faculty of the School of Engineering and Applied Sciences \\ University of Virginia \\ In Partial Fulfillment \\ of the Requirements for the Degree of \\ Master of Science in Mechanical \& Aerospace Engineering
}

By

Brian J. Connolly

December

2016 


\section{APPROVAL SHEET}

The thesis

Is submitted in partial fulfilment of the requirements

For the degree of

Master of Science

Brian Connolly

Author

The thesis has been read and approved by the examining committee:

Dr. Eric Loth

Advisor

Dr. Christopher Goyne

Dr. C. Frederic Smith

Dr. Houston Wood

Accepted for the school of Engineering and Applied Science:

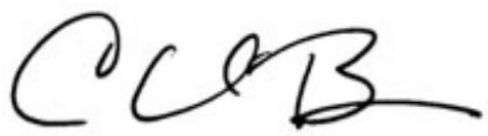

December

2016 
An Inertial Particle Separator (IPS) is a particulate removal device typically installed at the inlet of a gas turbine to mitigate effects of sand ingestion on the engine. This system can minimize particulate ingestion during helicopter landings in austere brown-out conditions so as to increase engine life. Typically, IPS systems have lower engine power losses than alternative engine inlet filtration technologies.

The present studies investigated a variety of novel IPS geometries. Tests were conducted on a fundamental twodimensional experimental facility that allows optical access. Geometries were evaluated using a variety of performance criteria. Of primary import is particle separation efficiency which measures the effectiveness of the system at removing particulate from the core engine flow stream. The separation efficiency of a particular IPS configuration is reliant in part on the characteristics of the flowfield, especially for finer particulate. Low scavenge air mass flow fractions are desirable, as it is directly related to the amount of power diverted from the engine to power the IPS. However, lowering scavenge mass flow fraction increases rates of flow separation and instability, leading to lost particle separation efficiency. There is a lack of available experimental data for IPS geometries designed to operate effectively at these low scavenge mass flow fractions.

Modifications from a baseline geometry were made to the scavenge leg and Outer Surface Geometry (OSG). Performance was evaluated based on particle separation efficiency, Particle Image Velocimetry (PIV), surface flow visualization, first order predictive methods, as well as power loss and mass flow rate variations. These experiments showed that reductions in the scavenge channel height were effective at increasing separation efficiency at low scavenge mass flow fraction. Additionally, these modifications successfully reduced flow instability and demonstrated a link between flow separation and particle separation efficiency. The present studies provide comprehensive empirical data to guide future development of IPS geometries specifically optimized to perform effectively at low power levels. 


\section{Table of Contents}

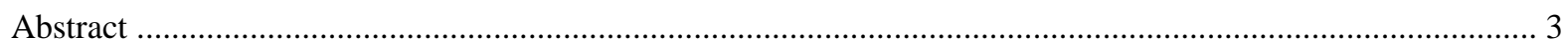

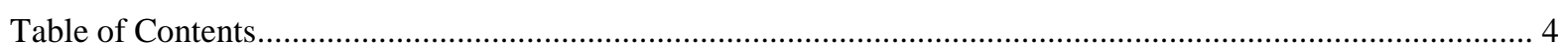

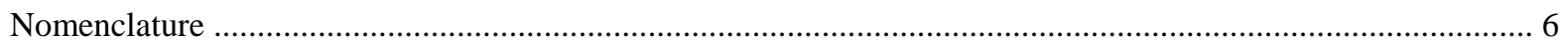

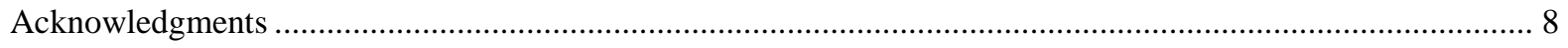

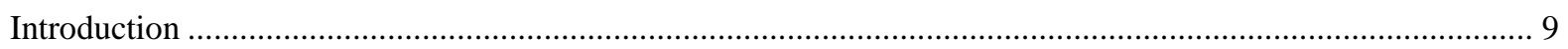

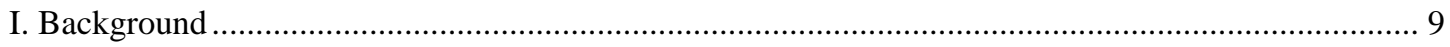

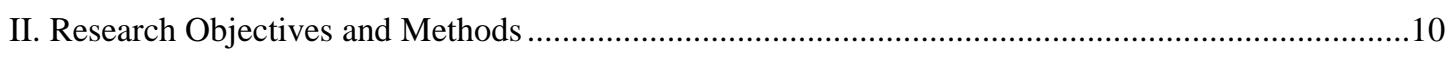

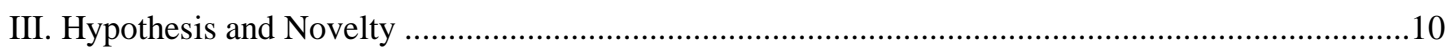

1: Influence of Scavenge Leg Geometry on Inertial Particle Separator Performance ...................................12

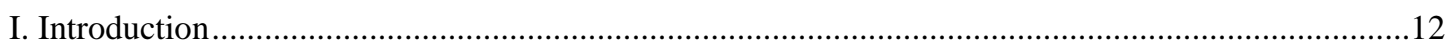

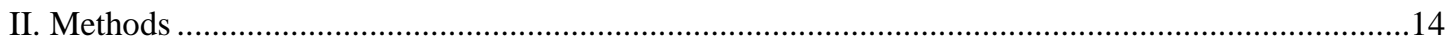

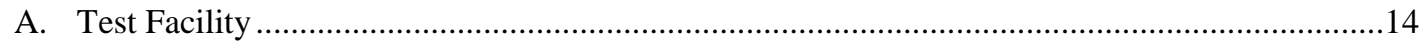

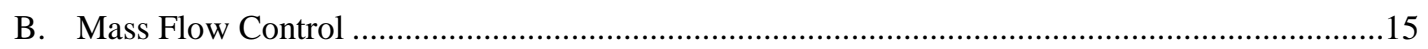

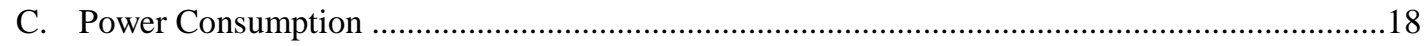

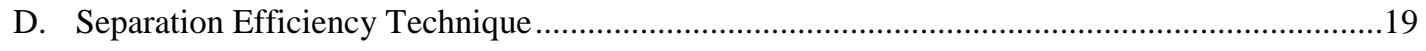

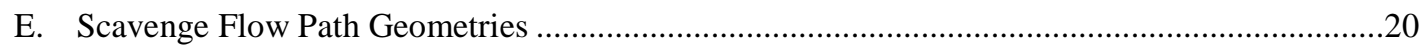

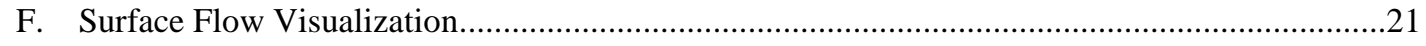

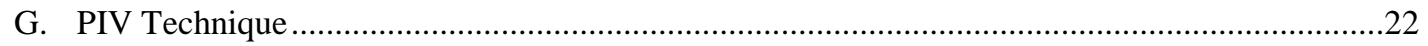

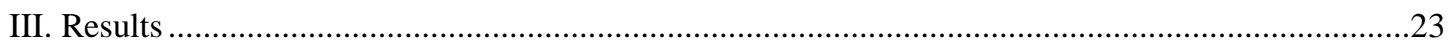

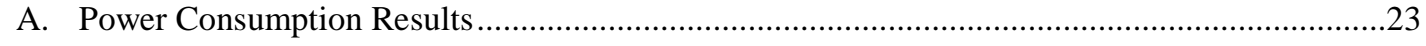

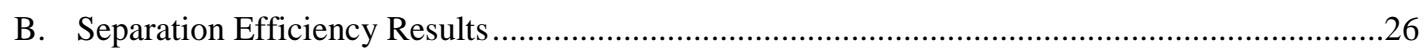

C. Surface Flow Visualization Results ................................................................................28

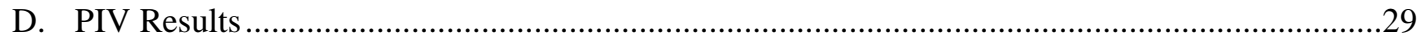

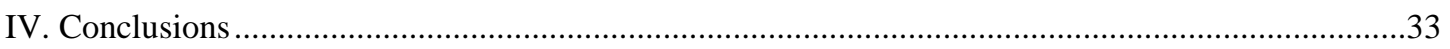

2: Novel Outer Surface Geometry for an Inertial Particle Separator .............................................................35

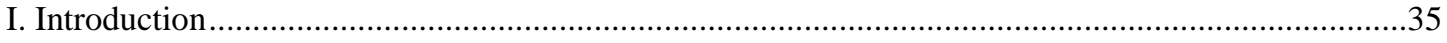

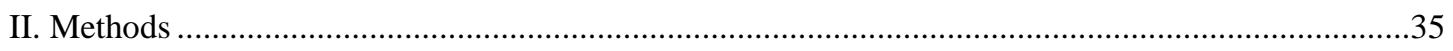

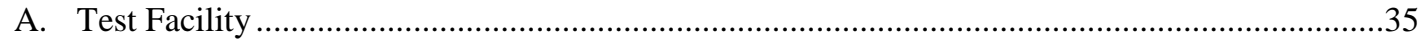

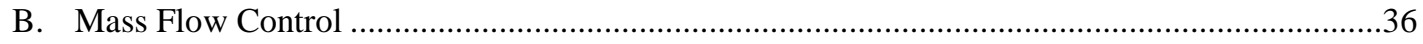

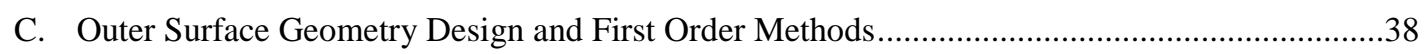

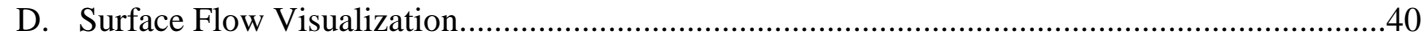

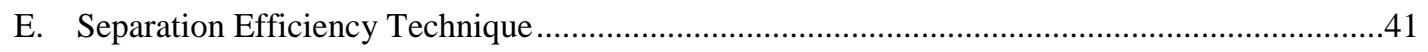

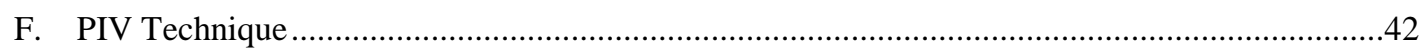

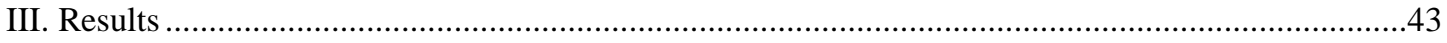
A. Surface Flow Visualization Results ................................................................................4

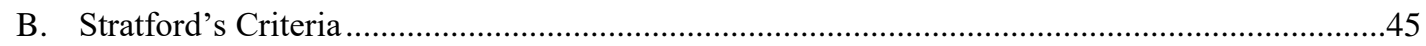




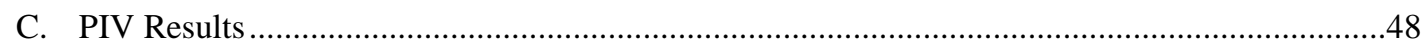

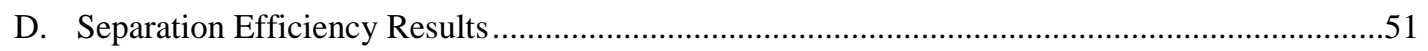

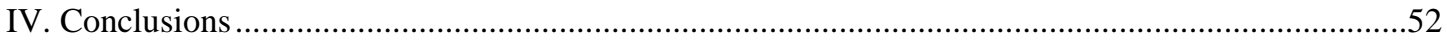

3: Parametric Study of Scavenge Channel Height in an Inertial Particle Separator ...........................................54

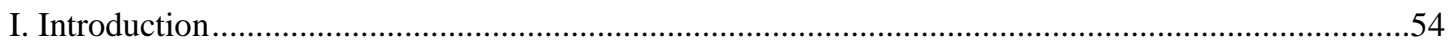

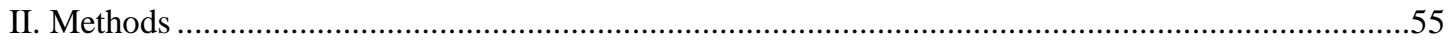

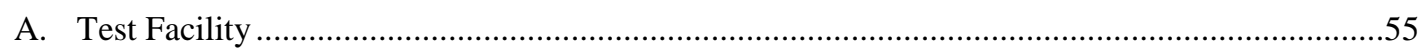

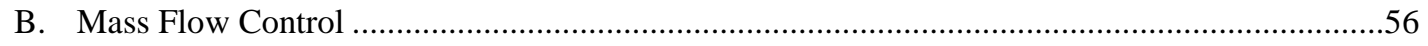

C. Inner Surface Geometry Design and First Order Methods ........................................................56

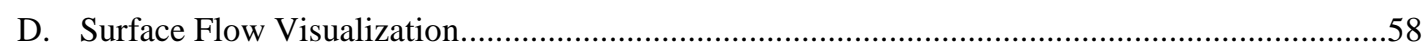

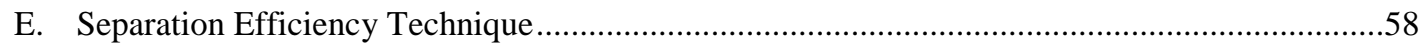

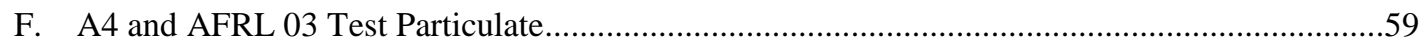

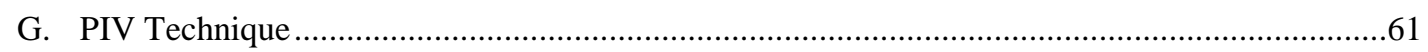

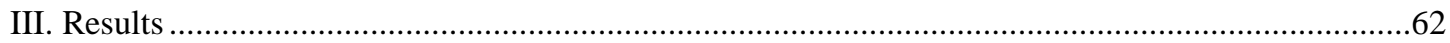

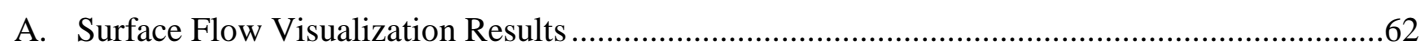

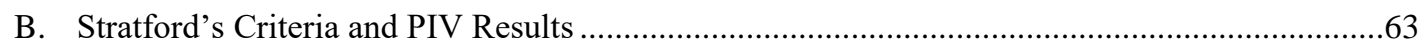

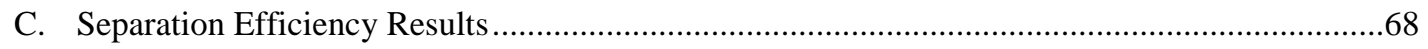

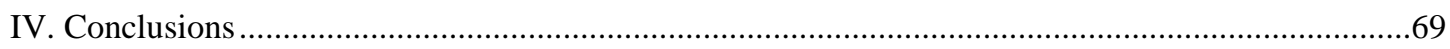

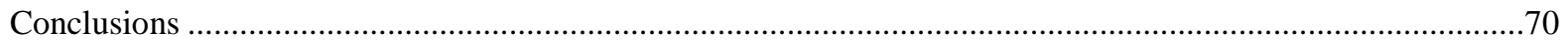

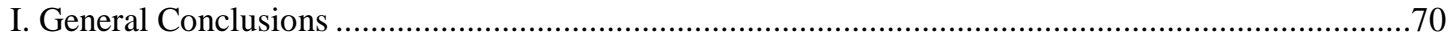

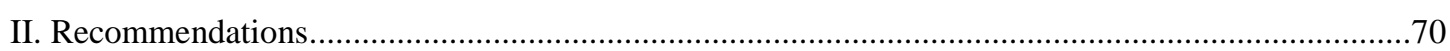

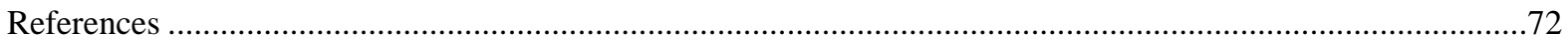




\section{Nomenclature}

\begin{tabular}{|c|c|c|}
\hline C & $=$ & coefficient \\
\hline$e$ & $=$ & error term \\
\hline$H$ & $=$ & throat height \\
\hline$K$ & $=$ & controller gain \\
\hline$L$ & $=$ & length of separation region \\
\hline$\dot{m}$ & $=$ & mass flow rate \\
\hline $\mathrm{N}$ & $=$ & number of image pairs \\
\hline$N$ & $=$ & motor speed \\
\hline$p$ & $=$ & pressure \\
\hline$R H$ & $=$ & relative humidity \\
\hline$s$ & $=$ & scavenge channel height \\
\hline $\mathrm{St}$ & $=$ & Stokes number \\
\hline$T$ & $=$ & temperature \\
\hline$t$ & $=$ & time \\
\hline$u$ & $=$ & controller output \\
\hline$v$ & $=$ & velocity \\
\hline $\mathrm{X}$ & $=$ & axial distance \\
\hline $\mathrm{y}$ & $=$ & normal distance \\
\hline
\end{tabular}

\section{Greek}

$\beta=$ scavenge mass flow fraction

$\Delta \quad=$ change of a quantity

$\delta=\quad$ error due to uncertainty

$\eta=$ particulate separation efficiency

$\rho \quad=$ density

$\sigma \quad=$ standard deviation 


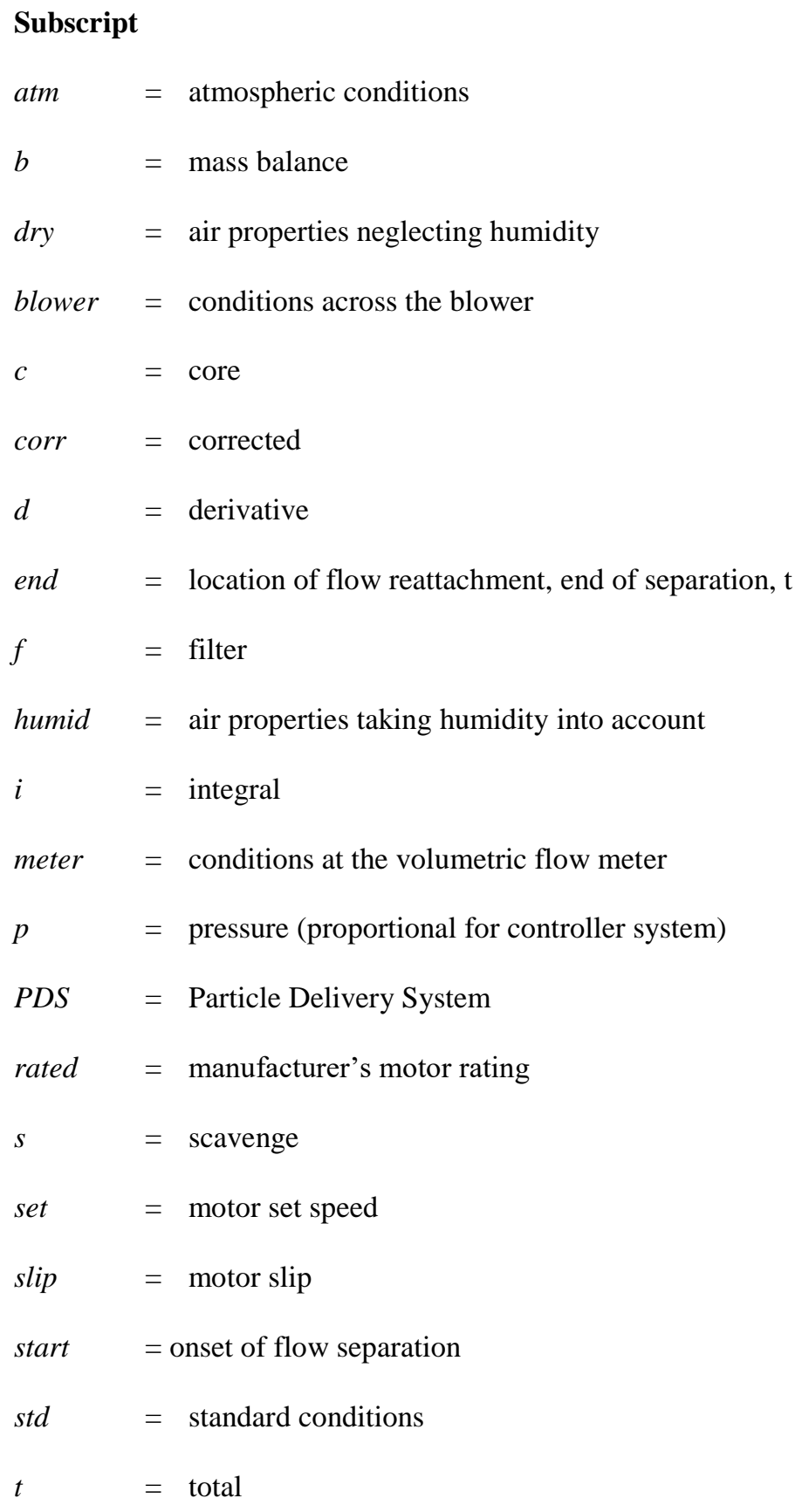




\section{Acknowledgments}

I would like to acknowledge the assistance of numerous family, friends, and colleagues in the completion of this thesis, without whom it could not have been completed. Thanks especially go to my advisor Dr. Eric Loth, who has invested considerable and sincere effort in my development as an engineer. I owe a great deal to Dr. Phil Snyder, Dr. Fred Smith, and Vic Oechsle of Rolls-Royce, as they have diligently provided a level of professionalism that I aspire to, as well as an enviable level of technical knowledge and, most of all, patience. Thanks also goes to undergraduate assistants Keegan Hammerton Devin Guthrie, and especially Matthew Rines, who worked many long hours and provided an excellent sounding board in support of this thesis. Finally, a big thank you to my parents, I am forever grateful for your love and support. This work was supported by the State of Virginia and Rolls-Royce North American Technologies, Inc. 


\section{Introduction}

\section{Background}

For gas turbine engines operating in conditions where sand and dust is prevalent, engine components are often subjected to serious damage, causing reduced engine life and increasing the potential for early removal. ${ }^{1-5}$ The U.S. Department of Defense Future Vertical Lift program ${ }^{6}$ identified "extreme environmental conditions" and higher than anticipated wear as major factors in increasing operational cost. An Inertial Particle Separator (IPS) can significantly reduce engine wear with minimal power loss by removing the majority of the particles before the airflow is delivered to the engine. Such a device uses a curved, bifurcating flowpath at the engine inlet to both reflect larger particles and concentrate smaller particles into the scavenge region and away from the engine core flow stream. In an operational IPS, scavenged particles are then removed via the scavenge flow while the cleaned air proceeds to the engine core. The fundamental goal of such systems is to separate as much particulate as possible at the lowest achievable scavenge mass flow rates while achieving low engine power losses.

While such flow paths are generally axisymmetric, a similar two-dimensional path was investigated herein allowing detailed optical access to the velocity field. Contaminated air enters the separator and makes a turn away from the hub-side whereby the particles have sufficient inertia to move outward to the Outer Surface Geometry (OSG). The flow then encounters the splitter where the particle-laden flow on the outside of the turn is scavenged away from the clean air nearer the hub-side. For typical test dusts, the largest of the particles have high inertia and thus are not significantly influenced by the airflow via particle drag. Their path through the separator is instead predominantly governed by incoming particulate trajectories and the resulting reflections or bounces on the IPS flowpath surfaces. ${ }^{7}$ Therefore, the hub, splitter, and outer surface geometry will generally be designed to ensure that such bouncedominated particles generally enter the scavenge flow leg at a wide range of operating conditions. ${ }^{8}$ For the smaller drag-dominated particles, the scavenge air mass flow is important to pull these particles away from the clean leg. These two regimes can be quantified by the Stokes number (St), a dimensionless quantity measuring how closely a particle will follow a flow field. ${ }^{10}$ Bounce-dominated particles have a large Stokes number $(\mathrm{St}>>1)$ while dragdominated particles have a small stokes number $(\mathrm{St}<<1)$. 
Modern IPS systems typically utilize scavenge mass flow rates (defined as the mass fraction of air entering the scavenge leg relative to the total mass flow rate of air) of $15-20 \% \%^{2,9,10}$ resulting in particle separation efficiency (defined as mass fraction of particulate removed by the scavenge leg relative to the total mass of particulate ingested) on the order of $80-90 \%$ or higher for A4 (coarse) test dust.

Recent work by Barone et al. ${ }^{10-12}$ determined that flow unsteadiness in the region of the flowpath bifurcation plays a substantial role in separation efficiency. An understanding of both the airflow patterns and the particulate motions in this region is therefore critical. These studies suggest that unsteadiness due to flow separation in the IPS bifurcation region has a deleterious effect on particle separation efficiency.

\section{Research Objectives and Methods}

The studies presented herein seek to expand on the work of Barone by investigating novel IPS geometries. Specifically, this work focuses on IPS particle separation for geometries specifically designed to operate at lower power settings than conventional designs. Of particular interest are the flowfields associated with the new geometries, and the flow instability therein. Ideal geometries would separate the maximum amount of particulate with minimum system power costs.

These objectives are evaluated by a number of experimental methods. Surface flow visualization is used to characterize flow separation in the IPS. Particle Image Velocimetry affords instantaneous and time-averaged views of the flowfields. System power consumption is monitored for all operating conditions. Most critically, particle separation efficiency is measured for each geometry over a variety of flow conditions.

\section{Hypothesis and Novelty}

The following work seeks to evaluate the hypothesis of whether changing the IPS geometry through a series of modifications can adapt existing designs to operate at lower power consumption levels. The general reasoning of these modifications can be simplified to an effort to reduce flow separation without harmfully affecting particle trajectories. The correlation of flow unsteadiness and loss of particle separation efficiency observed by Barone is interrogated further with a variety of flowpath modifications.

The specifics of these new designs are detailed in their specific chapters, along with a theoretical framework for understanding these results in the context of the IPS design space. The majority of flow contractions do, in fact, act to 
reduce flow separation, and particle separation efficiency is improved for a number of the new designs. This reinforces the idea that flow instability and particle separation efficiency are closely linked, validating the hypothesis of the work.

To the author's knowledge, these are the first studies openly published about these novel IPS geometries. Each geometry was thoroughly evaluated using the methods described above. Improvements to IPS power consumption and particle separation efficiency were observed, with theoretical understandings offered. 


\section{1: Influence of Scavenge Leg Geometry on Inertial Particle}

\section{Separator Performance}

\section{Introduction}

For gas turbine engines operating in conditions where sand and dust is prevalent, engine components are often subjected to serious damage, causing reduced engine life and increasing the potential for early removal. ${ }^{1-5}$ The U.S. Department of Defense Future Vertical Lift program ${ }^{6}$ identified "extreme environmental conditions" and higher than anticipated wear as major factors in increasing operational cost. An Inertial Particle Separator (IPS) can significantly reduce engine wear with minimal power loss by removing the majority of the particles before the airflow is delivered to the engine. Such a device uses a curved, bifurcating flowpath at the engine inlet to both reflect larger particles and concentrate smaller particles into the scavenge region and away from the engine core flow stream. In an operational IPS, scavenged particles are then removed via the scavenge flow while the cleaned air proceeds to the engine core. The fundamental goal of such systems is to separate as much particulate as possible at the lowest achievable scavenge mass flow rates while achieving low engine power losses.

While such flow paths are generally axisymmetric, a similar two-dimensional path was investigated herein allowing detailed optical access to the velocity field. A schematic of the present two-dimensional IPS geometry is shown in Figure 1.1a. Contaminated air enters the separator and makes a turn away from the hub-side whereby the particles have sufficient inertia to move outward to the Outer Surface Geometry (OSG). The flow then encounters the splitter where the particle-laden flow on the outside of the turn is scavenged away from the clean air nearer the hubside. For typical test dusts, the largest of the particulates have high inertia and thus are not significantly influenced by the airflow via particle drag, so that their path through the separator is instead predominantly governed by incoming particulate trajectories and the resulting reflections or bounces on the IPS flowpath surfaces. ${ }^{7}$ Therefore, the hub, splitter, and outer surface geometry will generally be designed to ensure that such bounce-dominated particles 
generally enter the scavenge flow leg at a wide range of operating conditions. ${ }^{8}$ For the smaller drag-dominated particles, the scavenge air mass flow is important to pull these particles away from the clean leg. Modern IPS systems typically utilize scavenge mass flow rates (defined as the mass fraction of air entering the scavenge leg relative to the total mass flow rate of air) of $15-20 \%{ }^{2,9,10}$ resulting in particle separation efficiency (defined as mass fraction of particulate removed by the scavenge leg relative to the total mass of particulate ingested) on the order of 80$90 \%$ or higher for A4 (coarse) test dust. The work reported here focuses on scavenge mass fractions near $10 \%$ with similar target values of particle separation efficiency.

Recent work by Barone et al. ${ }^{10-12}$ determined that flow unsteadiness in the region of the flowpath bifurcation plays a substantial role in separation efficiency.

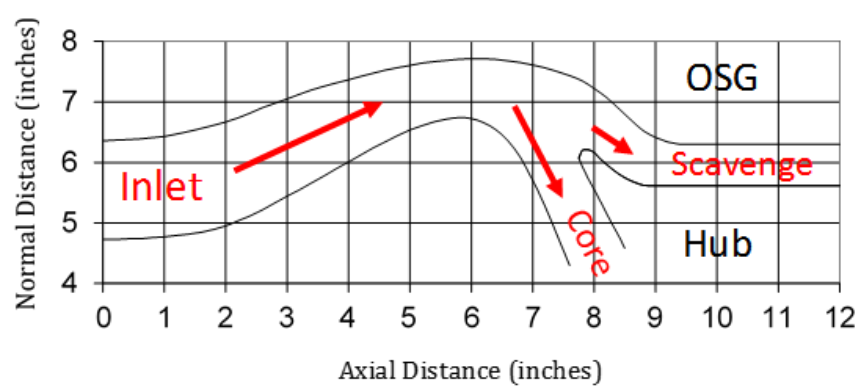

(a)

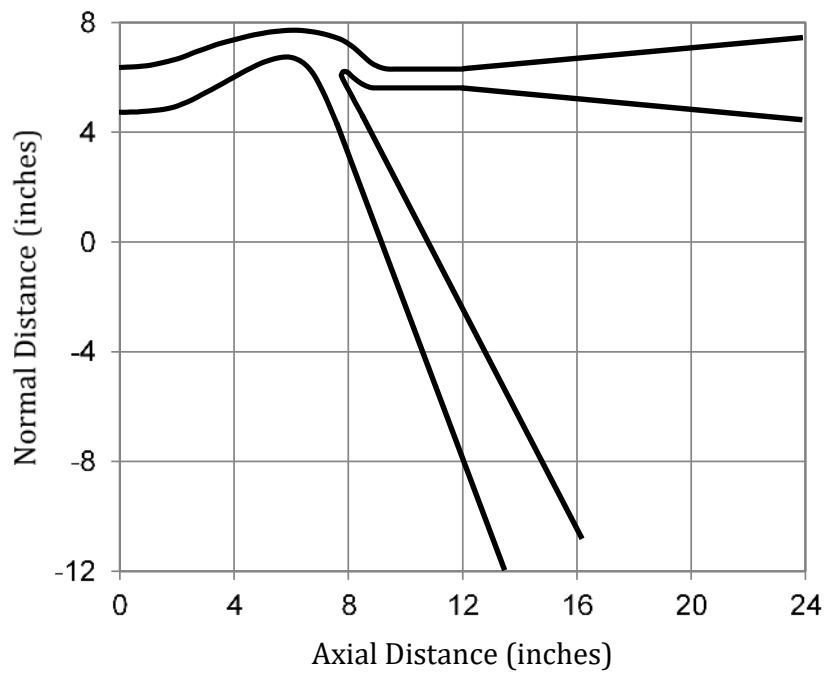

(b)

Figure 1.1. Schematics of the University of Virginia Inertial Particle Separator (IPS) flow path: (a) zoomed-in portion showing the test section, where particulate-laden airflow enters at the inlet, then undergoes a contraction and bifurcation so that particulate is ejected out the scavenge leg while the cleaned air flows through the core leg to the engine, (b) zoomed-out portion showing the downstream diffusers for the core and scavange legs. The depth (normal to the page) is a constant 8 inches throughout the test section of a), but the diffusers in b) have a decreasing depth.

Hence an understanding of both the airflow patterns and the particulate motions in this region can be critical in achieving designs that maximize particle separation efficiency. In this work it was found that the flow steadiness and power cost of the IPS to the engine is directly influenced by the fraction of scavenge mass flow rate of air. Higher scavenge airflow rates remove more particles from the core stream, but at the cost of greater power used to move the scavenge flow. 
The objective of the work reported here is to improve this understanding of the multiphase flow field with respect to changes in scavenge flow path geometry immediately downstream of the splitter nose, especially as it relates to the particle separation efficiency at low levels of scavenge mass fraction. Such an investigation has not been previously conducted to the author's knowledge. The current study aims to investigate this influence using the University of Virginia fundamental two-dimensional experimental facility which allows detailed optical access.

\section{Methods}

\section{A. Test Facility}

Tests are conducted in a wind tunnel facility at the University of Virginia ${ }^{8}$ (Figs. 1.1 and 1.2). The test section is a full-scale version of the flow path elements of an IPS using similar geometry converted from a two-dimensional axisymmetric geometry. By ensuring full-scale flow path dimensions in a span wise cut and full flow velocities, there was no need to employ Reynolds, Mach, or Stokes number scaling, assuming standard sea-level conditions. To eliminate gravitational bias normal to the flow for the particle trajectories, a vertical pull-down wind tunnel is used. Upstream of the wind tunnel entrance, the particles are dispersed to dilute conditions by compressed air ejection, which prevents undesirable clumping. The resulting particle cloud simulates high particle loading that can occur during austere helicopter landing conditions. The particulate then enters the present wind tunnel facility through an inlet with a flow straightener and a smooth contraction into the test section, where it bifurcates into the scavenge and

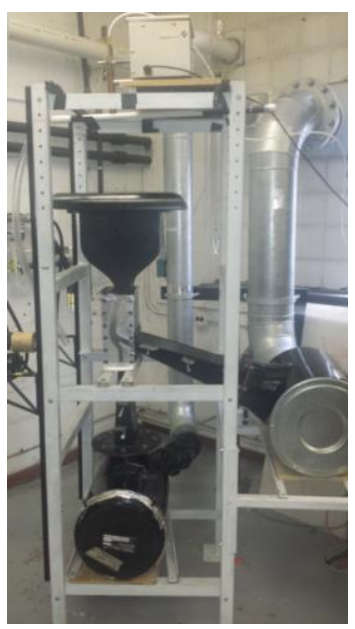

(a)

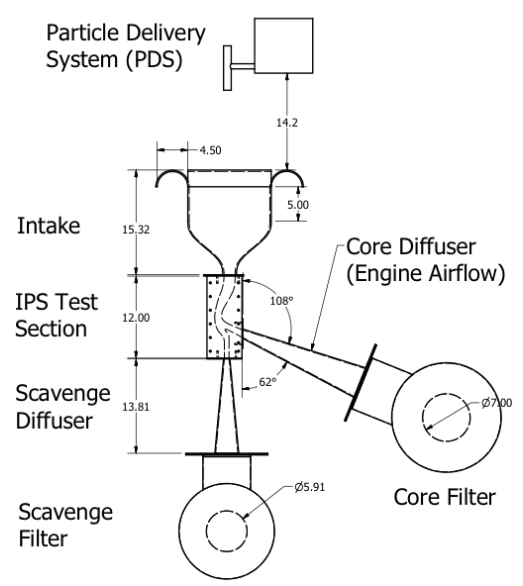

(b)

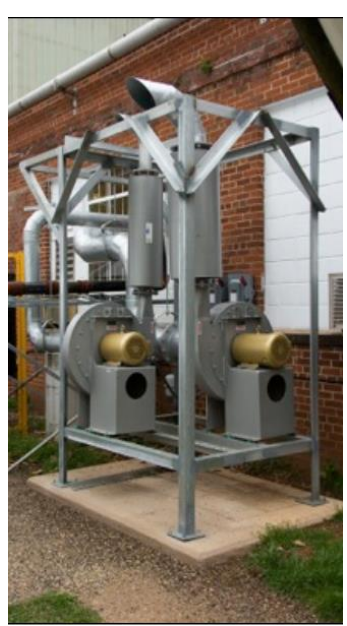

(c)

Figure 1.2. Photographs of the University of Virginia Inertial Particle Separator Facility: (a) vertical tunnel, including inlet, test section, and filter housings where test section of Figure 1.1 is oriented so flow is downward, (b) tunnel schematic (inches). and (c) external blower configuration for both scavenge and core streams. 
core legs. Air mass flow rate and pressure loss through the scavenge leg and the core leg are directly tied to the power consumption of the blowers used in the test system. Diffusers in each leg (scavenge and core) connect the tunnel to air filters, which collect the test dust to allow separation efficiency measurements. The scavenge leg diffuser has a rather aggressive expansion of 10.6 degrees, a length of 13.81 inches, and initial hydraulic diameter of 1.262 inches. Downstream of the filters, the flow in each leg follows a duct system to its respective blower motor and is then exhausted into ambient (outdoor) conditions.

\section{B. Mass Flow Control}

The scavenge mass flow fraction $(\beta)$ is defined as the ratio of scavenge leg mass flow rate $\left(\dot{m}_{s}\right)$ compared to the total inlet mass flow rate, which is the sum of the mass flow rates in the core leg $\left(\dot{m}_{c}\right)$ and scavenge leg $\left(\dot{m}_{s}\right)$, i.e.

$$
\beta=\frac{\dot{m}_{s}}{\dot{m}_{S}+\dot{m}_{c}}
$$

The flow is driven by exhaust fans that are controlled to achieve the proper $\beta$ values. For the core leg, a $15 \mathrm{hp}$ Cincinnati Fan HP-8D22 is used with a rated maximum volumetric flow rate of 1600 SCFM (Specific Cubic Feet per Minute), and the scavenge leg employs a 7.5hp Cincinnati Fan HP-4C22 with a rated maximum volumetric flow rate of 640 SCFM.

During a typical test, the core volumetric flow rate is held ideally constant at 1390 CFM (Cubic Feet per Minute), a value consistent with equivalent mass flow rates per unit span of an actual engine. Various values of $\beta$ are then obtained by varying the scavenge leg volumetric flow rate. Volumetric flow rates in both legs are measured by TekAir VT5000 VorTek airflow measurement system insertion probes (Fig 1.3a). The probes register vortex shedding frequency to obtain the velocity, within a stated accuracy of $2 \%$. The velocity is multiplied by the calibrated area of the duct to obtain the volumetric flow rate. The power of the blower motors and the atmospheric temperature and pressure conditions in the lab is recorded at the time of the test.

Determining the mass flow rate from the volumetric flow rate requires knowing the air density at the flow measurement stations, which are located downstream of the filters and upstream of the blowers. Since the blowers discharge directly to the atmosphere, the pressure at the flow measurement stations can be estimated using the pressure rise curves for standardized conditions provided by the blower manufacturer. This introduces an unknown level of uncertainty to the mass flow data. The speed of the blowers during the test is estimated by subtracting a slip speed $\left(N_{\text {slip }}\right)$ from the set motor speed $\left(N_{\text {set }}\right)$. This is divided by the rated speed $\left(N_{\text {rated }}\right)$, and the quantity is squared in order 
to scale the pressure rise according to standard fan laws. The standardized pressure rise is then adjusted to the atmospheric conditions of the test by the density ratio of the atmospheric air and the standard conditions given by the manufacturer:

$$
\Delta p_{\text {blower }}=\Delta p_{\text {rated }}\left(\frac{N_{\text {set }}-N_{\text {slip }}}{N_{\text {rated }}}\right)^{2} \frac{\rho_{\text {atm }}}{\rho_{\text {rated }}}
$$

In this expression, $\dot{m}$ is the mass flow, $\mathrm{p}$ is the pressure, and $\rho$ is the air density.

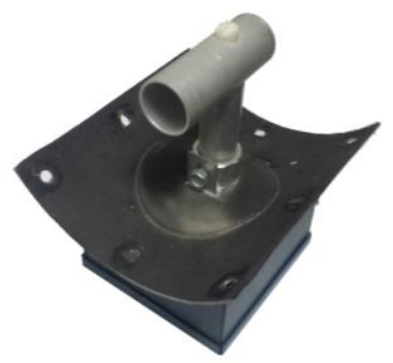

(a)

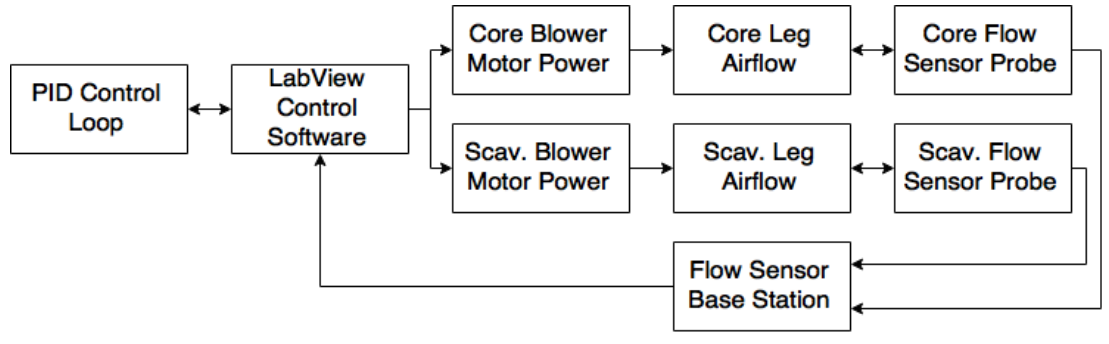

(b)

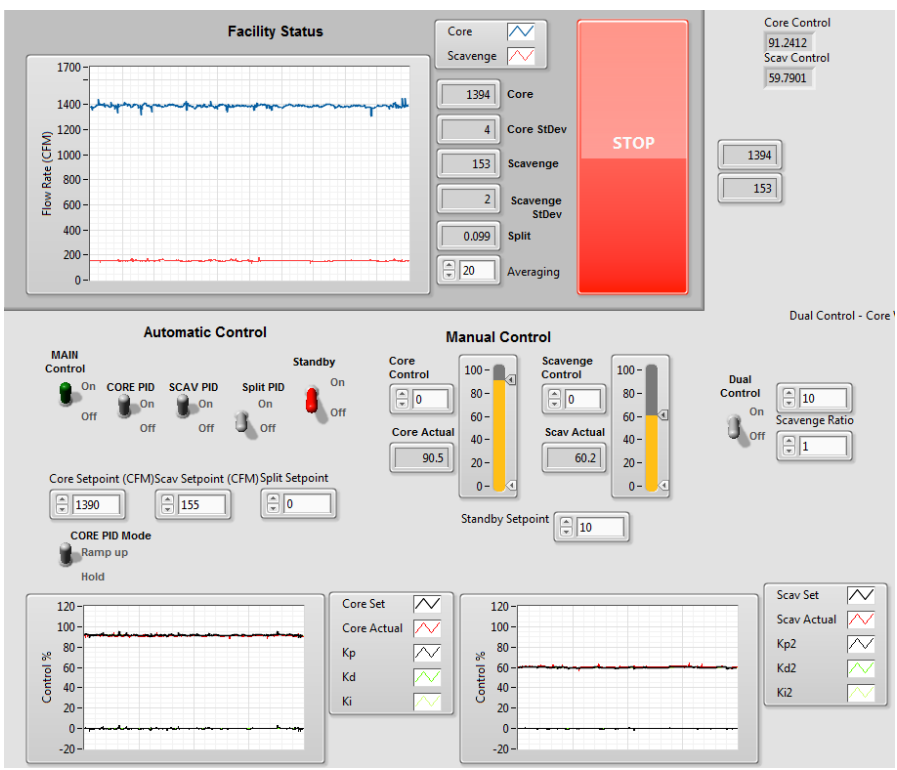

(c)

Figure 1.3. Mass flow Control Feedback Loop. (a) Tek-Air VT5000 VorTek airflow measurement system insertion probe. (b) Tunnel control system schematic, c) Tunnel control panel includes mass flow rates (top) in CFM, PID system settings (middle), and motor power percentages (bottom). 
From this, the air pressure at each of the flow measurement stations is obtained by using atmospheric conditions modified by the expected pressure rise across each respective blower:

$$
p_{\text {meter }}=p_{\text {atm }}-\Delta p_{\text {blower }}
$$

Future improvements to the tunnel include direct pressure monitoring at each flow sensor for real-time pressure calculation, obviating the need to calculate the pressure after the test. To compute air density at each of the measurement stations, the local pressure (from Eq. 3) is combined with local temperature and humidity (estimated as equal to ambient conditions) to obtain a moist air density based on the IUPAC 1981 standard $^{14}$. Mass flow for each leg is then obtained by multiplying the calculated air density at the meter and the measured volumetric flow at the meter. The mass flow in each leg is then corrected to standard conditions to maintain Mach number consistency in the test section for all tests. The correction given by Mattingly ${ }^{15}$ is employed with an additional correction for humidity as follows:

$$
\dot{m}_{\text {corr }}=\frac{\dot{m}_{\text {meter }} \sqrt{\frac{T_{a t m}}{T_{\text {corr }}}}}{\frac{\rho_{\text {atm }, \text { humid }}}{p_{\text {corr }}}} \frac{\rho_{\text {atm, dry }}}{\rho_{\text {atr }}}
$$

In practice, the two blowers do not operate independently, and adjusting the settings of one requires a simultaneous change in the operating point of the other. Changes to the flow path geometry and air filter particulate loading have significant effects on the pressure losses and thus the required power for each motor. In order to allow rapid convergence to the selected motor powers and $\beta$ value, the system is controlled automatically as shown in Figure 1.3b. This is accomplished through the inclusion of LabView's Proportional-Integral-Derivative (PID) closed loop control system for each of the motors in the blower control program. It operates on the following principle:

$$
u(t)=K_{P} * e(t)+K_{i} \int_{0}^{t} e(t) d t+K_{d} * \frac{d e(t)}{d t}
$$

In this expression, $t$ is the current time, $u(t)$ is the controller output, $e(t)$ is the error term (i.e. the difference between the target flow rate and the measured flow rate from the probe), and the $\mathrm{K}_{\mathrm{p}}, \mathrm{K}_{\mathrm{i}}$ and $\mathrm{K}_{\mathrm{d}}$ are the Proportional, Integral, and Derivative (PID) controller gains, respectively. The core gain settings were tuned to values of $\mathrm{K}_{\mathrm{p}}=0.042$ and $K_{i}=0.0105$, while the scavenge gain settings were $K_{p}=0.06$ and $K_{i}=0.04$. The scavenge gains were higher because the motor is smaller and more responsive to power changes, and so could be more quickly adjusted. Due to the overall slow response time of the blower motors, no derivative term is needed for either motor in the control program $\left(K_{d}=0\right)$. Over the course of five minutes of stable operation at $\beta=16 \%$, this PID controller reported mean 
volumetric flow rates to be within $0.3 \%$ of the target, and the recorded standard deviations of the flow rates were $\leq$ $1 \%$ of the total air flow rate. The control panel for testing is shown in Figure 1.3c.

The uncertainty of the scavenge mass flow fraction, $\delta \beta$, is conducted per Barone et al. ${ }^{8}$ :

$$
\delta \beta=\sqrt{\left(\frac{\dot{m}_{s}}{\left(\dot{m}_{s}+\dot{m}_{c}\right)^{2}} \delta \dot{m}_{c}\right)^{2}+\left(\frac{\dot{m}_{S}}{\left(\dot{m}_{S}+\dot{m}_{c}\right)^{2}} \delta \dot{m}_{s}\right)^{2}}
$$

The analysis uses the error in each of the legs, $\delta \dot{m}_{c}$ and $\delta \dot{m}_{s}$, taken as the $2 \%$ reading error reported in the flow meter documented calibration. The values of $\delta \beta$ for all the tests used in this study were about $2 \%$.

\section{Power Consumption}

One of the major benefits of an IPS over similar technologies is its relatively low engine power requirement while removing as much harmful particulate from the engine flowpath as possible. As such, a modification to the flow geometry must be judged in terms of both particle separation and total system power costs. Modifications to the scavenge leg geometry were analyzed for their effect on core and scavenge blower power consumption over a wide range of operating conditions. Due to the nature of the channel bifurcation, the core and scavenge power costs are interdependent. As will be shown, a change in the scavenge leg geometry can have a significant effect on power consumption of not just the scavenge blower, but the core as well.

In addition to minimizing power consumption, a successful geometry would be able to operate at a wide variety of conditions. By varying the scavenge motor power from $100 \%$ to its lowest possible value before flow reversal in the scavenge leg, the entire operating range of available $\beta$ is obtained. Since $\beta$ is closely analogous to the overall power costs of the system, a low minimum achievable $\beta$ is more relevant to this study than a high maximum achievable $\beta$.

For a power consumption test, clean air filters are installed in both core and scavenge legs to keep pressure losses downstream of the test section consistent between tests. The core PID controller is engaged, with a mass flow target of 1390 SCFM. The scavenge blower was set at $100 \%$ power and held for 20 seconds, during which the average flow rates in the core and scavenge are collected, as well as their motor powers. The process is repeated at various scavenge power settings while the core PID attempts to hold 1390 SCFM. Since the actual power consumption of the blowers

is affected by downstream diffusers, filter housing, and ductwork, these aspects were all kept consistent to allow a relative comparison between the various scavenge leg geometries. 


\section{Separation Efficiency Technique}

To directly measure the particle separation efficiency (by mass) of a given flowpath geometry, approximately $100 \mathrm{~g}$ of test dust is released into the inlet over a period of five minutes by a Particle Delivery System (PDS) volumetric feeder. The test particulate is A4 Coarse Test Dust (ISO 12103-1). This dust is composed mostly of silica $\left(\mathrm{SiO}_{4}\right)$ and aluminum oxide $\left(\mathrm{Al}_{2} \mathrm{O}_{3}\right)$ but also contains several other components in smaller quantities; it has a size distribution from 0.5 to 300 microns. Compressed air at 25 psi is used to scatter the particulate into a cloud similar to what could be expected for a helicopter landing in high particulate conditions. The compressed air has the added benefit of breaking up particle clumps that may have formed during the dispensing process. Clumping is important to avoid as it can skew the effective size distribution of the test particulate. Once through the test section, the dust is captured in the downstream filters. These filters, as well as the PDS, are weighed before and after each test for determination of the total mass of particulate used in the test. The separation efficiency $(\eta)$ is then calculated as the ratio of the mass gained in the scavenge filter $\left(\Delta m_{s f}\right)$ to the total mass gained by both the core filter $\left(\Delta m_{c f}\right)$ and the scavenge filter:

$$
\eta=\frac{\Delta m_{s f}}{\Delta m_{c f}+\Delta m_{s f}}
$$

For example, in the case where $90 \mathrm{~g}$ of the particulate is captured by the scavenge filter and $10 \mathrm{~g}$ by the core filter, $90 \%$ efficiency is achieved.

Mass discrepancies $\left(\delta m_{r}\right)$ can occur if particulate sticks to the sidewalls of the tunnel or passes through the filters. These are recorded for each test by comparing the mass change of the PDS ( $\Delta m_{P D S}$, net particles delivered) to the total mass change of the filters $\left(\Delta m_{c f}+\Delta m_{s f}\right.$, net particles collected):

$$
\delta m_{r}=\Delta m_{P D S}-\left(\Delta m_{c f}+\Delta m_{s f}\right)
$$

All efficiency test data in this report have less than $2 \%$ mass discrepancy. The balance on which the PDS and filters were weighed was a Sartorious Signum 1, with $0.1 \mathrm{~g}$ resolution and repeatability of $0.08 \mathrm{~g}$. This translated to a balance error, $\delta m_{b}$, of $<0.05 \%$. The uncertainty of the separation efficiency, $\delta \eta$, is conducted per Barone ${ }^{8}$ :

$$
\delta \eta=\sqrt{\left(\frac{\Delta m_{s f}}{\left(\Delta m_{c f}+\Delta m_{s f}\right)^{2}}\left(\delta m_{r}{ }^{2}+\delta m_{b}{ }^{2}\right)\right)^{2}+\left(\frac{\Delta m_{s f}}{\left(\Delta m_{c f}+\Delta m_{s f}\right)^{2}}\left(\delta m_{r}{ }^{2}+\delta m_{b}{ }^{2}\right)\right)^{2}}
$$

The values of $\delta \eta$ for all the tests used in this study were $<2 \%$. 


\section{E. Scavenge Flow Path Geometries}

Since the scavenge leg undergoes a rapid stream-tube diffusion (associated with a sevenfold decrease in velocity), it may be prone to flow separation and, hence, significant unsteadiness. This was found to cause unsteadiness in the overall mass flow rates particularly at low $\beta$ values. To reduce this scavenge leg unsteadiness, various area reductions were investigated, as shown in Figure 1.4. These reductions were made by inserting isolator ramps downstream of the splitter nose. These changes preserve the outer surface geometry upstream of the splitter nose so that upstream particle reflections by bounce-

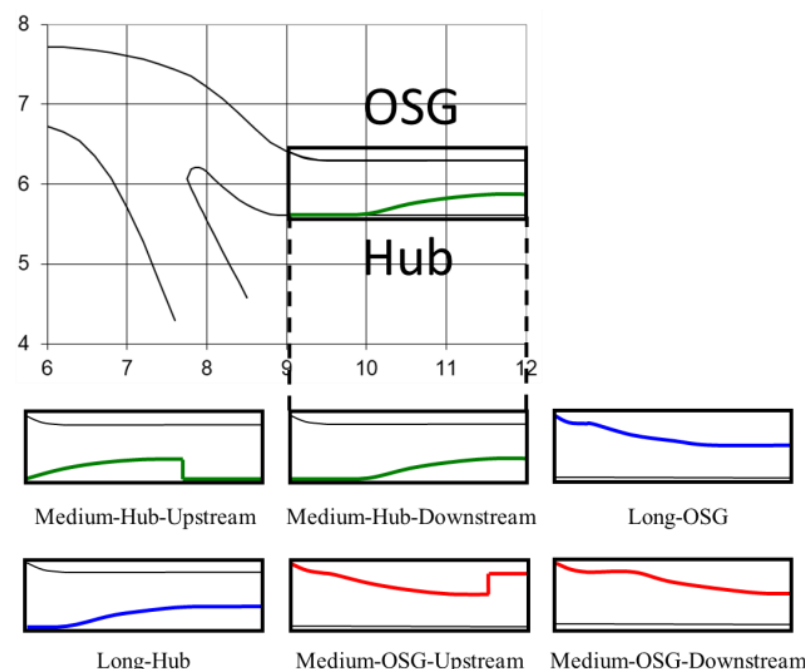

Figure 1.4. Schematic of isolator ramps used to modify scavenge leg geometry. Ramps are classified by size (medium or long), normal location (hub mounted or OSG mounted), and axial location (upstream or downstream). The ramps are defined as follows: Medium-HubUpstream, Medium-Hub-Downstream, Long-OSG, LongHub, Medium-OSG-Upstream, and Medium-OSGDownstream.

dominated particulate are not expected to be affected by this geometry change.

Various isolator ramps were tested and an associated classification system was defined based on length and location. Medium ramps extend 2" in the axial direction, while Long ramps extend 2.6" to create a smoother contraction of the cross-sectional area. All the ramp reported herein reduced the cross-sectional area of the scavenge leg by approximately one third (the scavenge path height was reduced from 0.685 " to 0.429 ") and extend the full 8 " width of the test section. Further area reductions (reducing the cross section by more than one half) were also tested but resulted in severely decreased efficiency and unacceptably high power consumption. As such, the results from those higher blockage tests are not reported herein.

The ramps are manufactured out of ABS plastic by a 3-D printer and are affixed tightly in the test section using silicone sealant (Fig. 1.5a). The condition without any ramps is termed the baseline geometry against which others are judged. This baseline geometry is approximately consistent with the previous OSG1 configuration by Barone et al. ${ }^{10}$. The Medium-Hub-Downstream case was also tested with an extended ramp that replaces the backward facing step with a smooth expansion (Fig. 1.5b). 


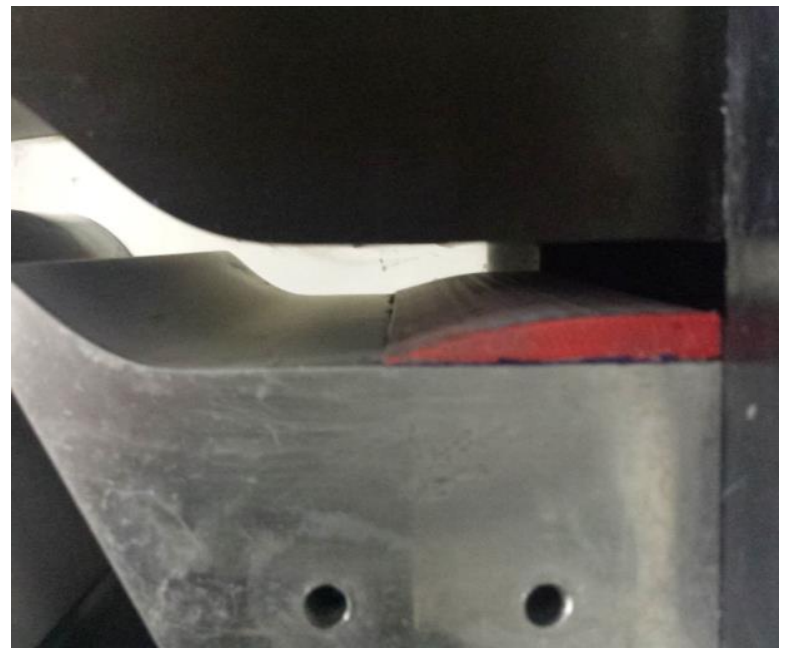

(a)

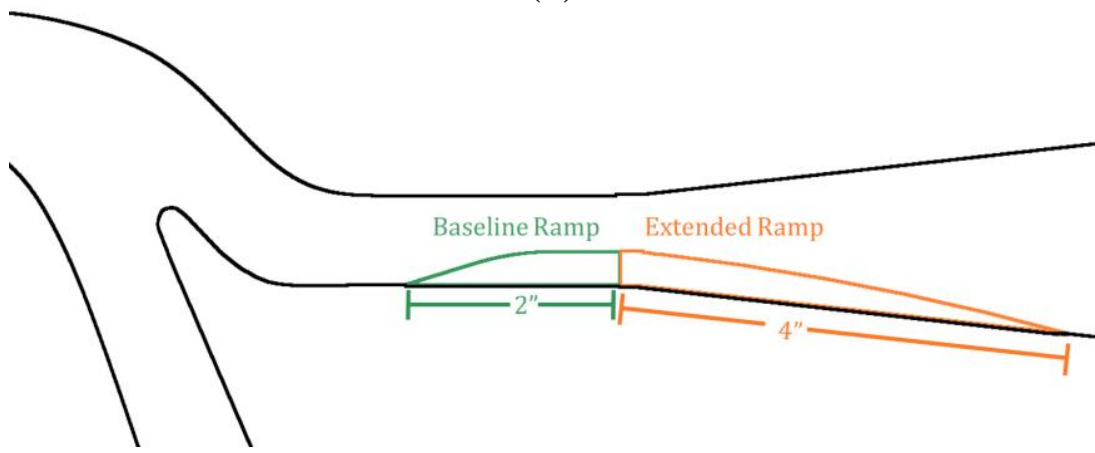

(b)

Figure 1.5. Isolator ramp installed in scavenge leg:. (a) photograph of ramp configuration with MediumHub-Downstream, and (b) schematic of Medium-Hub-Downstream Extended ramp which is includes a 4" extension into the scavenge diffuser.

\section{F. Surface Flow Visualization}

Surface flow visualization is used to characterize the large scale flow structures and separation ${ }^{16}$. The tests were conducted with titanium dioxide as the colorant, kerosene as the carrier fluid, and oleic acid as an emulsifier. The mixture was painted on the surface of interest just before operation and the flow then causes the suspension to streak, leaving a time-averaged wall shear pattern as the oil slowly dries. Note that the carrier fluid for such visualization is typically oil and not kerosene, but due to the highly unsteady nature of flow inside the IPS a more volatile fluid was chosen. Kerosene decreases the drying time significantly, allowing the resolution of more transient flow structures often present in separated flows. 
For a typical test, the titanium dioxide is mixed vigorously with the kerosene while a few drops of oleic acid is added. Once the suspension reaches an appropriate consistency, it is quickly applied to the OSG. Application is performed by a 2" painter's brush in strokes perpendicular to anticipated flow lines. This prevents false streamline identification due to the application technique. The OSG is then immediately reinstalled in the test section and the blowers are turned on. The surface is monitored to ensure the material has not dried before the tunnel reaches the desired operating point of $\beta=10 \%$. If this condition is met, the tunnel is left in operation for a minute, then the blowers are turned off and the OSG is removed for inspection. Care was taken to ensure that the kerosene did not evaporate before the target test condition was reached.

\section{G. PIV Technique}

Particle Image Velocimetry (PIV) is a method commonly used in gas flows to quantify the velocity field. It employs tracer particles seeded into the flow that are small enough in size so that they faithfully follow the surrounding airflow. The experimental approach used herein utilizes olive oil droplets of approximately $1 \mu \mathrm{m}$ diameter, because they very closely follow the flow due to their low Stokes number. These particles are illuminated with a laser sheet, and tracked using a camera according to the techniques as described here. The flows velocity field is calculated using a pair of captured images that contain the same set of particles after they have travelled a short distance. The field is subdivided into a discrete grid of sub-regions referred to as interrogation spots to obtain a two-dimensional matrix of velocity vectors. For each of these sub-regions, the particles that have traversed a small distance across two successive images are cross-correlated. The distance vector from the cross-correlation is divided by the time between the two images resulting in the velocity vector. By taking a large number of these image pairs throughout an experiment, an average flow field can be determined, along with the statistical variations present.

In the present set-up, the laser sheet is positioned at the centerline of the test section width so as to provide the clearest view of the flow at the bifurcation in order to characterize the flow in the region associated with the highest amounts of instability. The PIV camera is used to capture approximately 1000 image pairs, which are interrogated to examine instantaneous flow structures and velocity vectors. The camera records image pairs at a rate of $7 \mathrm{~Hz}$, which is sufficient for capturing instantaneous flow velocities and structures. This data was averaged to obtain the mean velocity and standard deviation of the velocity of the flow. This allows gas velocity to be measured within $2 \%$ accuracy. ${ }^{11}$ Further details of the PIV equipment and methods are described by Barone et al. ${ }^{11-12}$. 


\section{Results}

Investigation of the influence of the scavenge leg geometry on both the power required for scavenge and core mass flow rates as well as the separation efficiency, a number of controlled tests were completed on each of seven configurations as delineated in figure 4 plus the baseline No Ramp case. In addition a limited set of surface flow visualization tests were performed.

\section{A. Power Consumption Results}

In this series, the mass flow fraction $\beta$ was examined as a function of the scavenge motor power. Results are presented in Figures 1.6a and 1.6b. In each test the core volumetric flow was held constant via the PID controller to simulate engine operating conditions. It was found that for each of the scavenge leg geometries that there is a minimum achievable $\beta$ for each geometry, at which point any further reduction in scavenge motor power results in flow reversal and a total loss of separation efficiency. For the No Ramp case (baseline), the minimum $\beta$ was limited to about $8.5 \%$. All of the ramp configurations shown are found to have a lower minimum achievable $\beta$ than the No Ramp configuration. This indicates an increase in the useful operating range over that of the baseline configuration. In general, the Medium Ramp achieved the lowest $\beta$ values of about $6.5 \%$. As the scavenge power was reduced toward this minimum $\beta$ value, the power no longer decreases linearly and this corresponds to observed unsteadiness in the scavenge mass flow as measured by the volumetric meter.

The scavenge motor power vs. core motor power results are detailed in Fig. 1.6b. Lower core motor power is indicative of lower engine power penalties. The Medium-Hub-Downstream ramp and the No Ramp condition were found to required similar core motor powers. This power level was significantly less than the majority of the remaining ramp geometries. The Extended-Hub ramp had a similar minimum achievable $\beta$ and a comparable separation efficiency to the Medium-Hub-Downstream ramp. Note that these two configurations are similar. However, the extension also offers the lowest core motor power consumption of any ramp configuration. This is not unexpected in that the presence of the extension was expected to improve the performance of the downstream diffuser by replacing the backward facing step of the ramp with a smooth expansion. Again, the inflection point in the trend is observable at $60 \%$ scavenge motor power. Below this point, the core motor power does not continue to decrease. This suggests that unsteadiness in the test section is causing additional pressure losses that the core motor has limited capacity to overcome. 


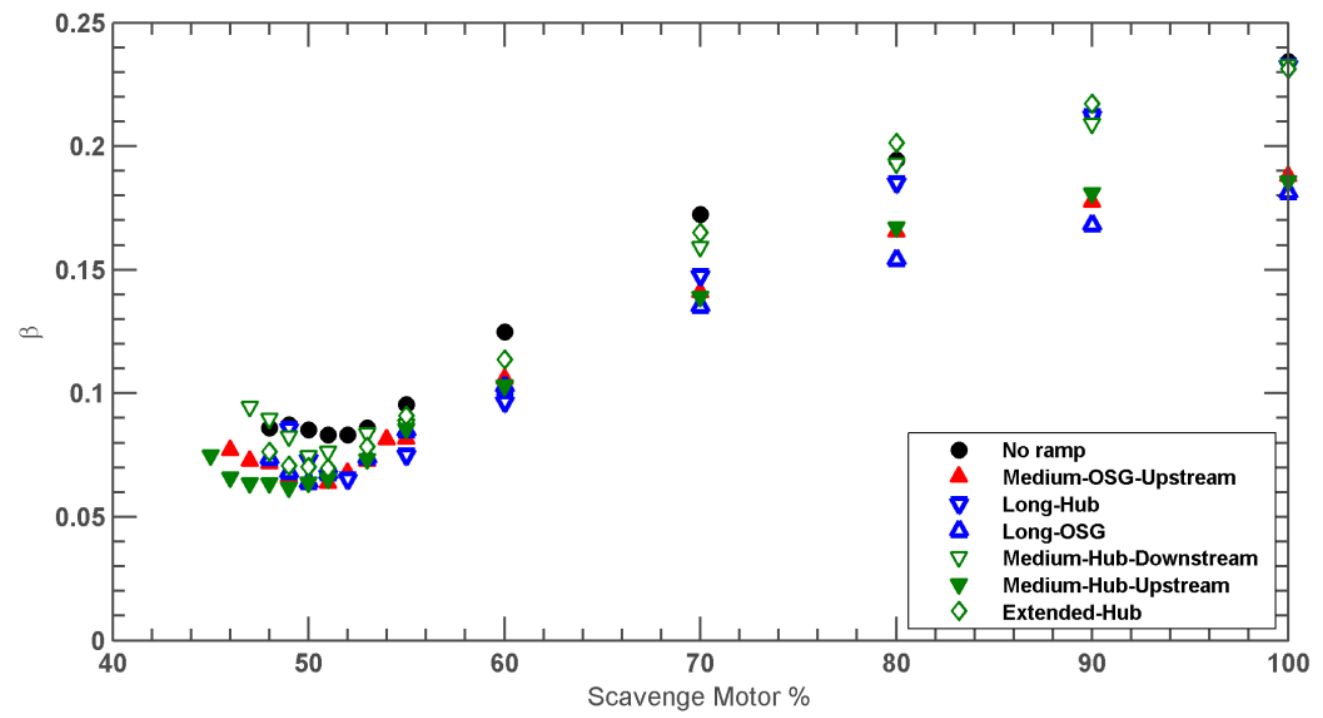

(a)

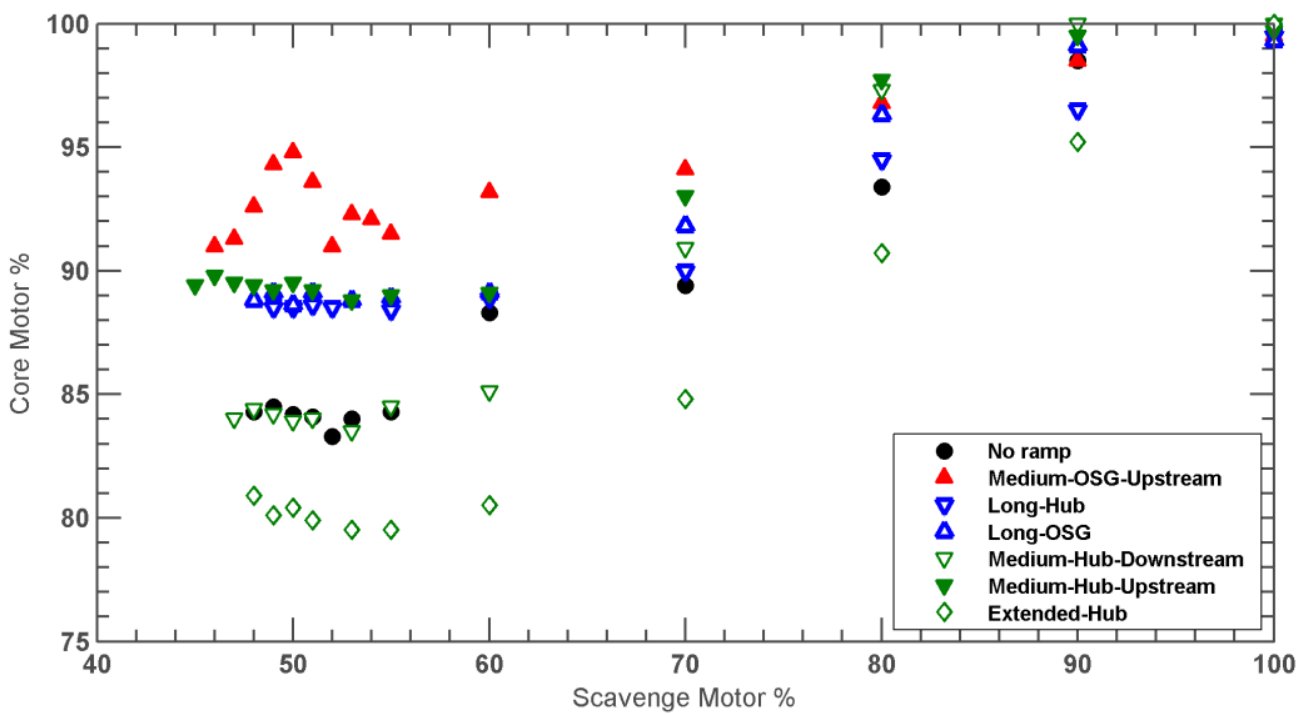

(b)

Figure 1.6. Influence of scavenge motor power on scavenge mass flow fraction. Scavenge motor power was set at $100 \%$ and the core motor was set by the PID to maintain a constant mass flow rate throughout the test. (a) $\beta$ as a function of scavenge motor power (b) Core motor power as a function of scavenge motor power. 
A characterization of this unsteadiness is shown in Fig. 1.7 for the No Ramp and the Medium-Hub ramp geometries. It can be seen that this ramp dramatically reduced the mass flow oscillations associated with flow unsteadiness when compared to the No Ramp condition. This result was consistent with a significant reduction in noise levels for the ramp case during flow operation, suggesting that this ramp in the scavenge leg reduces overall unsteadiness. Note that the time scale of the oscillations is a factor of the PID controller tuning, not the unsteadiness in the tunnel. At the peaks of the scavenge line color coded red in Figure 1.7a, the tunnel is operating with forward flow but the $\beta$ is higher than the set point. The PID attempts to correct this by lowering the scavenge power, but a flow reversal occurs and the flow rate then drops below the target rate. Thus it is found that there is no stable operating point at the desired $\beta$, so the PID is unable to hold that condition without oscillating. By contrast it is found that the ramp allows a much more stable operating condition and thus allowing a greater operational range of $\beta$.

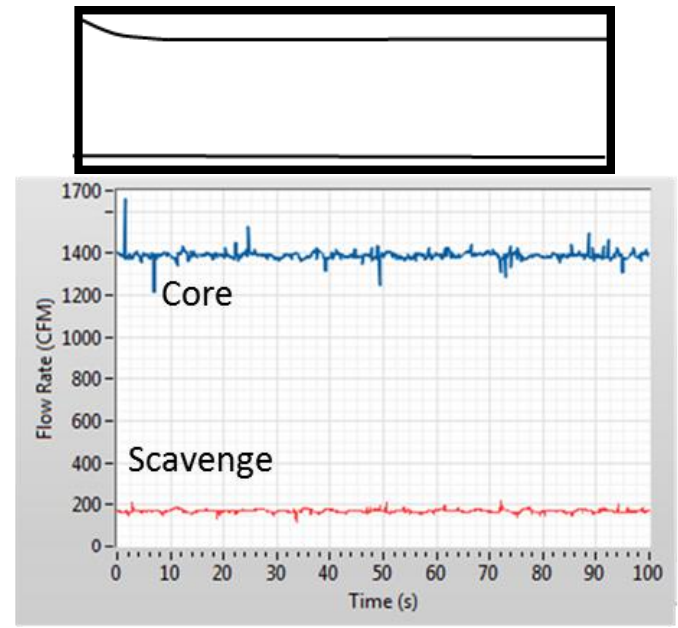

(a)

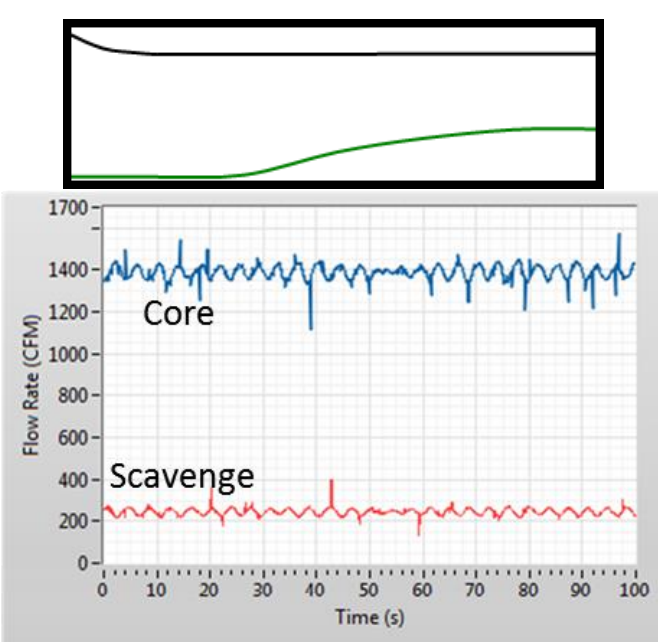

(b)

Figure 1.7. Mass flow variations. Core (blue) and scavenge (red) mass flow rates for: (a) No Ramp geometry showing increased unsteadiness for scavenge and even core flow, and (b) Medium-Sized Hub Ramp showing stable operation. 


\section{B. Separation Efficiency Results}

Separation efficiency tests at $\beta=10 \%$ are shown in Figure 1.8. The efficiencies are averaged results of 3 to 5 tests each, with less than $3 \%$ uncertainty in $\beta$, less than $2 \%$ uncertainty in $\eta$ for each individual test, and with less than a $2 \%$ difference in $\eta$ between tests at the same ramp condition. The variations of scavenge geometry create a substantial change in the particle separation efficiency. This is attributed to increased flow steadiness in the scavenge leg that is understood to cause the flowfield upstream of the splitter nose to also be more steady. This then allows higher particle separation levels to be experienced. Among the different geometries tested, the Medium-Hub ramps performed best in terms of maximizing $\eta$, followed by Long ramps (mounted on either the hub or OSG), with Medium-OSG ramps performing only marginally better than the No Ramp condition. Interestingly, Long ramps performed similarly whether placed on the hub or OSG.

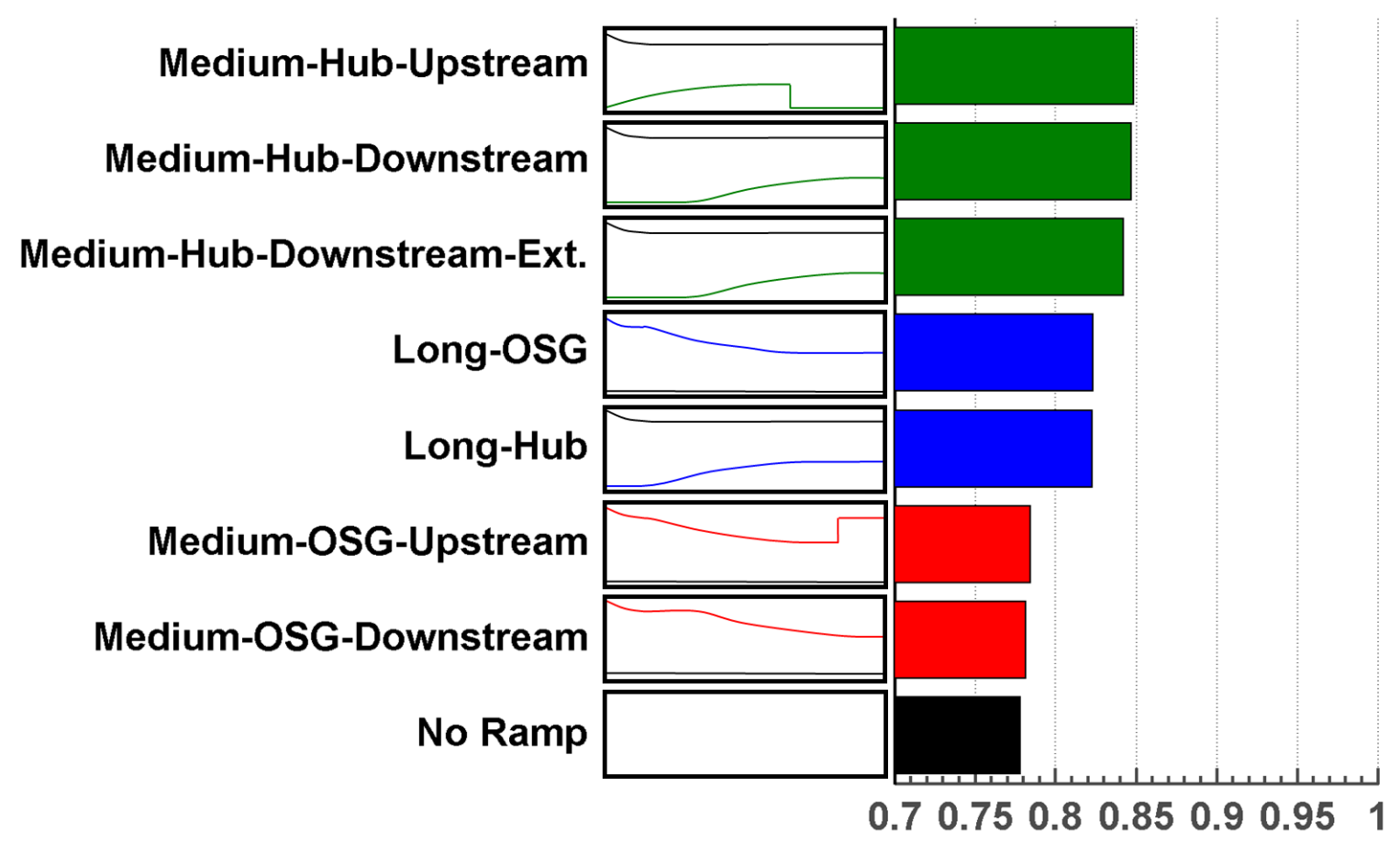

$\eta$

Figure 1.8. Average separation efficiency of individual ramps at a scavenge mass flow fraction of $\beta=$ $10 \%$. Actual mass flow rates are $\beta=10.3 \pm 0.1 \%$. 
The complete set of particle separation efficiency results for the range of $\beta$ investigated are presented in Figure 1.9. For the No Ramp configuration, there is a precipitous drop-off in efficiency around $\beta=10 \%$. However, the Medium-Hub-Downstream ramp continues to have good separation efficiency and low power requirements, for $\beta$ as low as $8 \%$. The Medium-Hub-Downstream ramp also exhibits this trend, but manages to outperform the No Ramp case by an even wider margin than at higher $\beta$. These results, taken with the data from the power consumption experiments, show that the ramps reduce the power required and the mass flow required for the same level of particle separation efficiency.

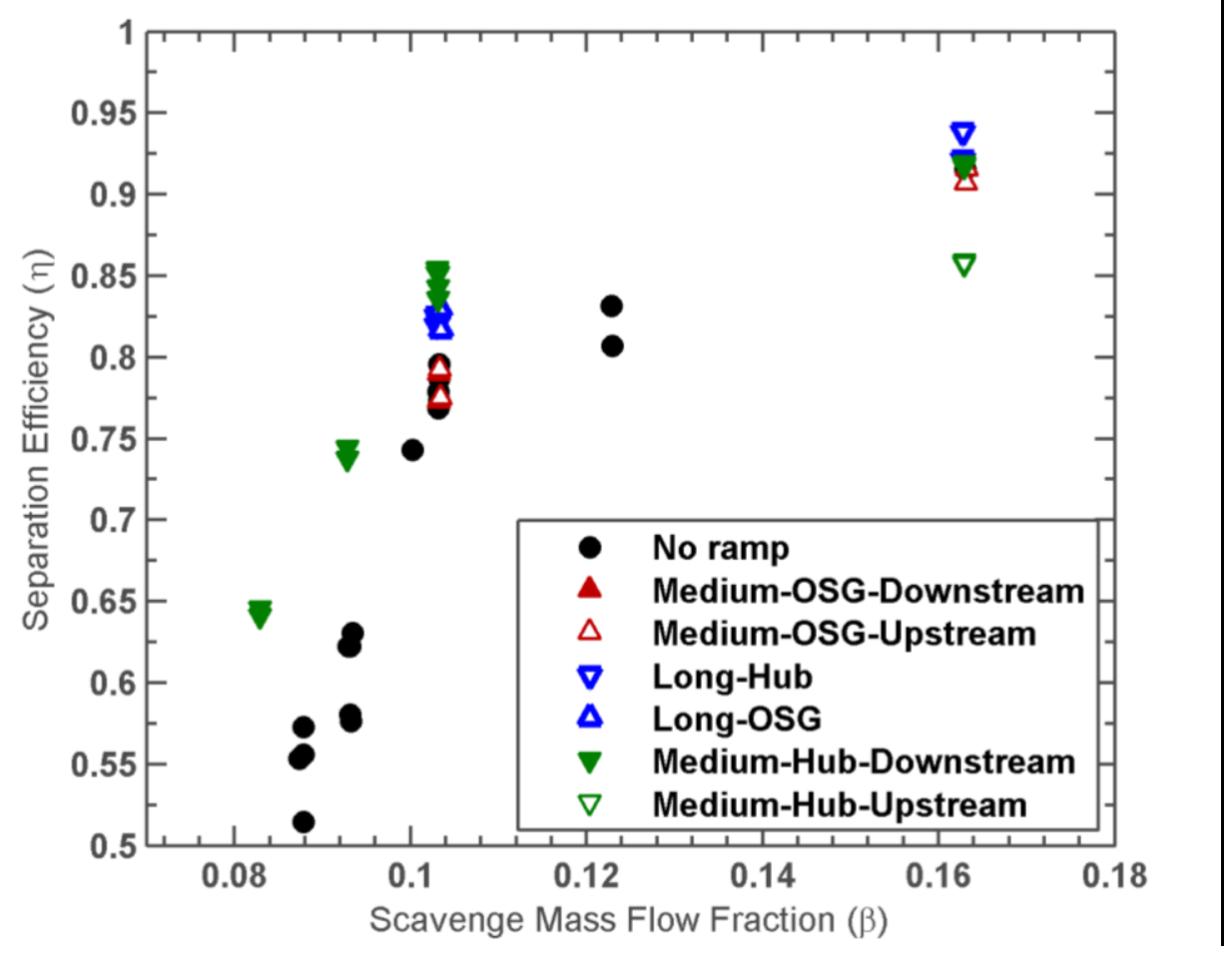

Figure 1.9. Separation efficiency of various types of ramps based on scavenge mass flow fraction. Testing focused on $\beta=10 \%$ and below as the ramps had little effect on separation efficiency at $\beta=16 \%$. Uncertainty for both $\beta$ and $\eta$ was within the size of the symbol in all tests shown. 


\section{Surface Flow Visualization Results}

Four surface flow visualizations were performed for this study for the Medium-Hub-Downstream extended case for $\beta=10 \%$. Three of the four appeared similar to Figure 1.10a. A clear but uneven line of separation was observed, with irregular structures downstream of the flow separation. The separation is approximately consistent along the span, with minor but noticeable sidewall separation. In contrast, the fourth result (Fig. 1.10b) displays a highly irregular separation with large, fully formed vortices present. This indicates that the flow was generally unsteady and three-dimensional. In Figure 1.10c, it can be seen that similar surface flow patterns were observed by Barone et al. $^{8}$

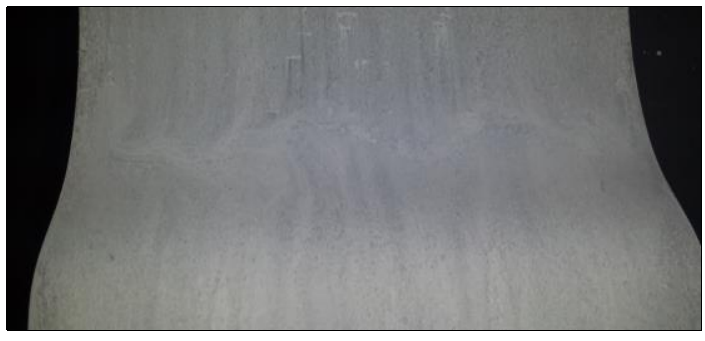

(a)

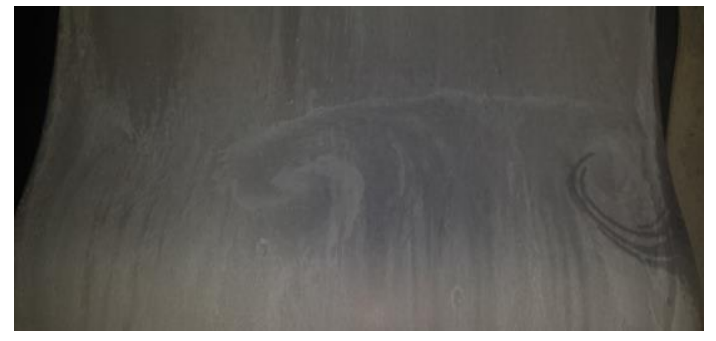

(b)

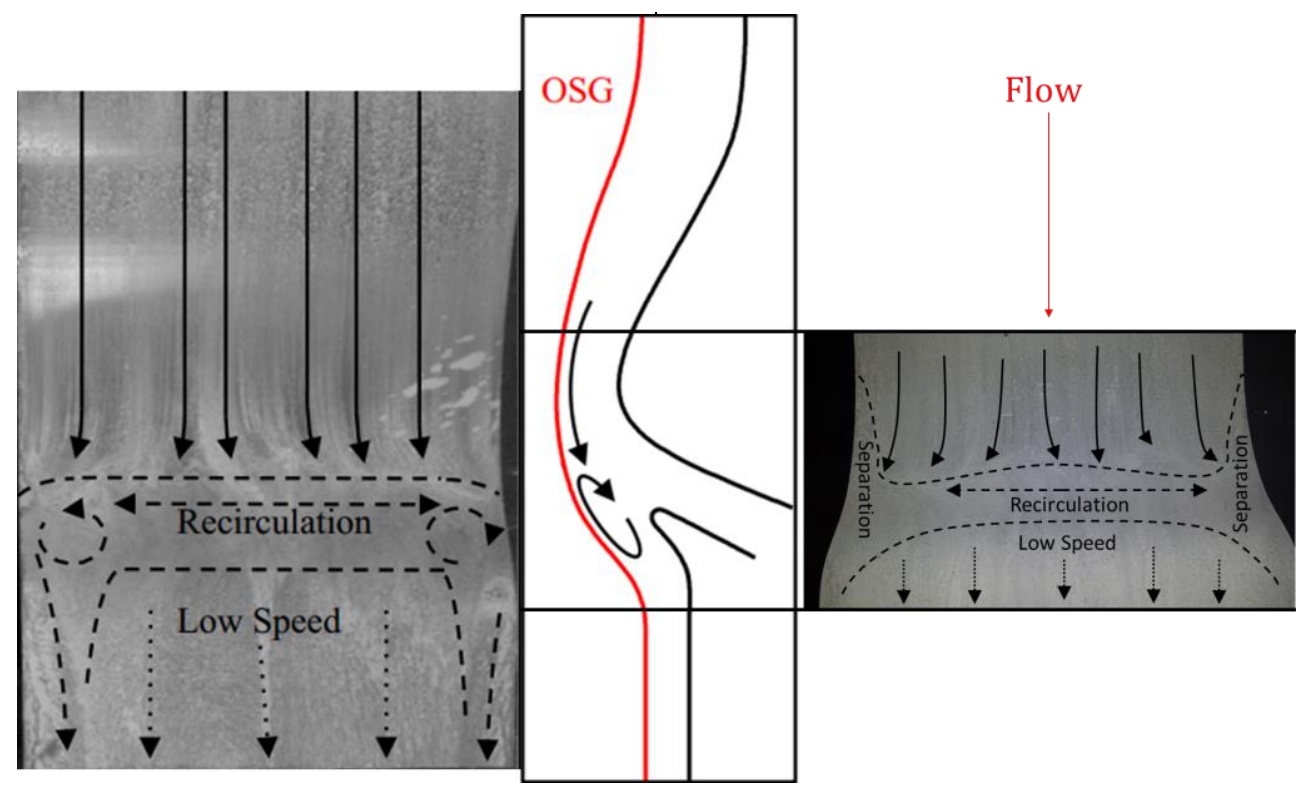

(c)

Figure 1.10. Oil Streak Surface Flow Visualization. Surface flow visualizations with Medium-Hub-Downstream ramp installed. Oil streak tests used a suspension of titanium oxide and oleic acid in kerosene. (a) Typical of most oil streak tests, with moderate, quasi-two-dimensional separation. (b) Rare capture of wall-normal vortex with fully three-dimensional separation. (c) Comparison between Barone $e t$ al. ${ }^{8}$ (no ramp) and typical oil streak results of current study. Similar separation lines were observed, but note increased sidewall separation in the current study. 
(left). The No Ramp and Medium-Hub-Downstream ramps exhibited the same overall behavior, but the ramp case flow visualization indicated a slight increase of sidewall separation.

Surface Flow visualization revealed the presence of irregular separation on the OSG side, with large, transient vortical structures for every case. Initial separation lines are markedly similar to results from Trünkle et al. ${ }^{16}$ In that study, separation on a smooth surface due to adverse pressure gradients was observed, at comparable Reynolds numbers to the present study. Separation was found to be highly unsteady, which again is consistent with these results.

\section{PIV Results}

The air velocity field was investigated with PIV for the Medium-Hub-Downstream extended ramp case and for the No Ramp case as shown in Fig. 1.11. While the mean flow fields are similar, the Medium-Hub-Downstream extended ramp results indicate an increased inflow velocity at the centerline (Fig. 1.11a). Since the mass flow rates are the same, this suggests that the isolator ramp may cause to an increase in sidewall separation, increasing centerline velocities (which is consistent with the flow visualization results). This result is a potentially unfortunate but expected consequence of removing some of the spanwise separation. Note that the mean flow entering the scavenge leg is more uniform for the ramp case, while that entering the core flow tends to have an increased velocity near the splitter nose. The standard deviation of the velocity (Fig. 1.11b) indicates significantly lower fluctuation levels for the ramp case, especially for the outer portion of flow entering the scavenge leg. This is consistent with steadier overall flow noted in Fig. 1.7, albeit the changes are larger for the overall volumetric flow rate which indicates the primary changes in unsteadiness are likely that in the scavenge diffuser. It is worth recalling that this diffuser had a somewhat aggressive expansion angle, and thus it is expected that the ramp prevent strong flow separation from occurring in the diffuser.

The instantaneous PIV plots (Fig. 1.12) show the wide variability in the flow streamlines within the test section indicating strong unsteadiness, even with the ramp installed. The flow fields in Fig. 1.12a and Fig. 1.12b were selected among the thousand image pairs in order to show the two important flow conditions. The flow paths of Figure 1.12a are instantaneous realization when there is little flow reversal (at least along the centerline where the measurements were taken). This type of flow is ideal for particle removal into the scavenge leg since all the streamlines are directed downstream, and with somewhat less separation for the Medium-Hub-Downstream ramp. In contrast, the instantaneous realization streamlines associated with Figure $1.12 \mathrm{~b}$ are problematic for particle removal since they indicate reverse flow regions near the splitter nose. This reingestion flow structure is observed in both with and without scavenge ramps, but occurred somewhat less frequently for the flow with the ramp installed. The challenge for future 

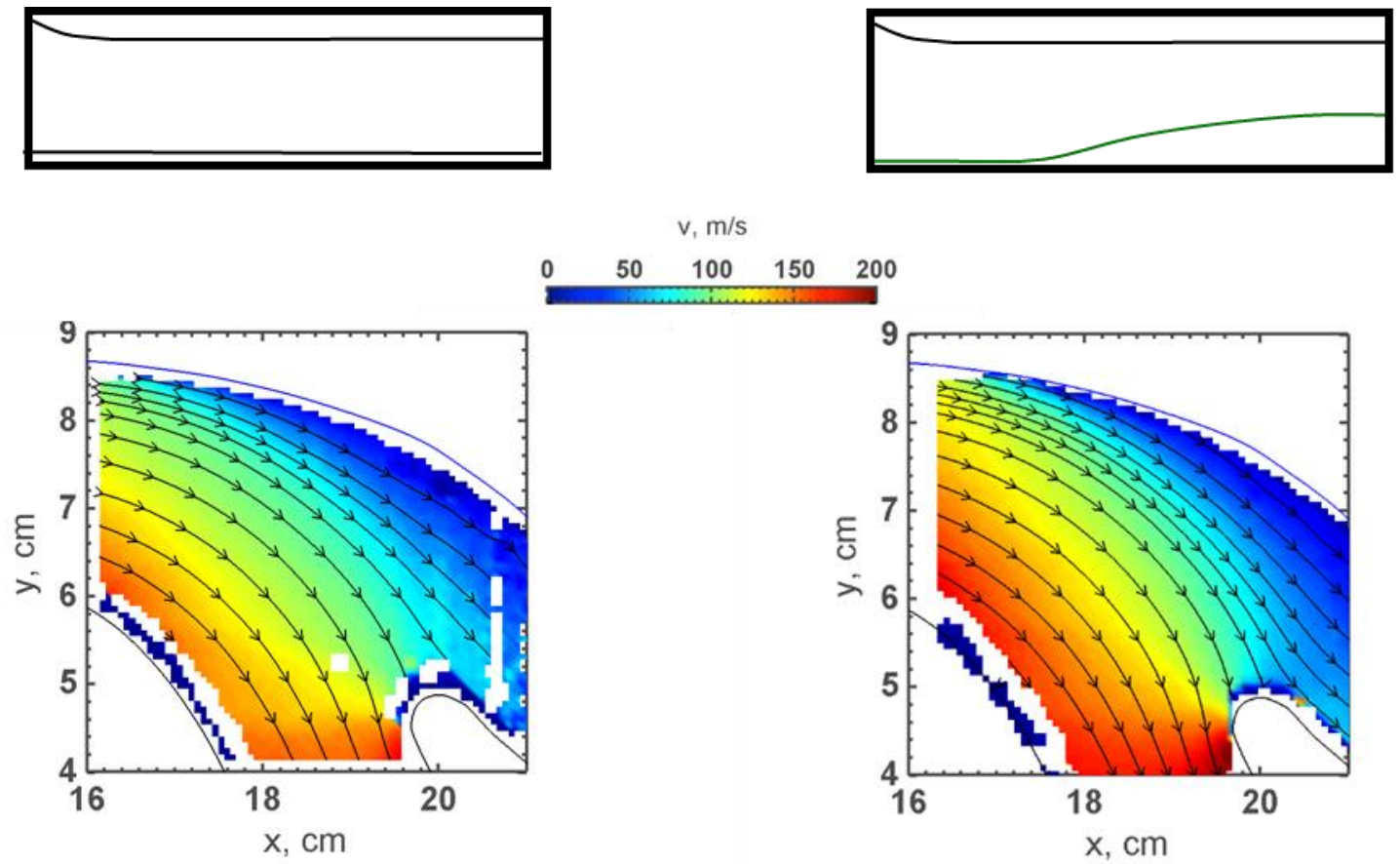

(a) Time-Averaged Velocity

$\sigma, \mathrm{m} / \mathrm{s}$

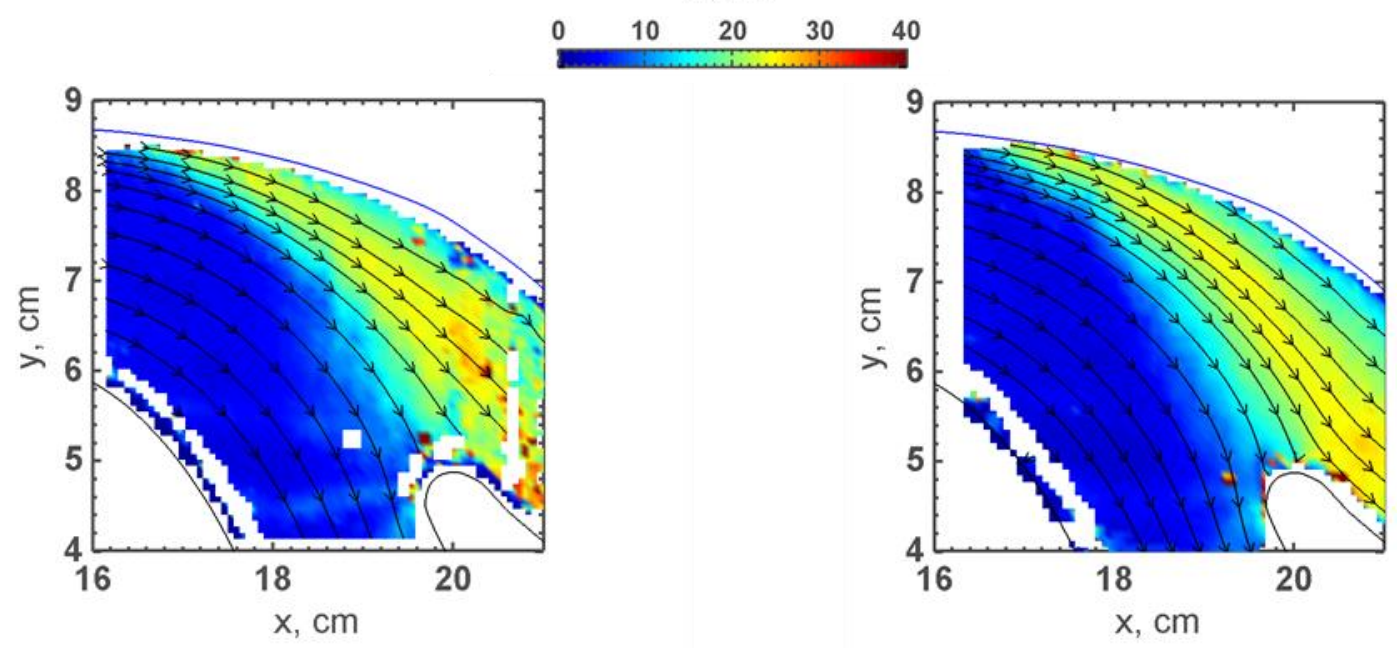

(b) Time-Averaged Velocity Standard Deviation

Figure 1.11. PIV Data at $\beta=10 \%$ with no ramp (left) and the Medium-Hub-Downstream ramp (right). Actual mass flow rates are $\beta=10.3 \pm 0.1 \%$. 

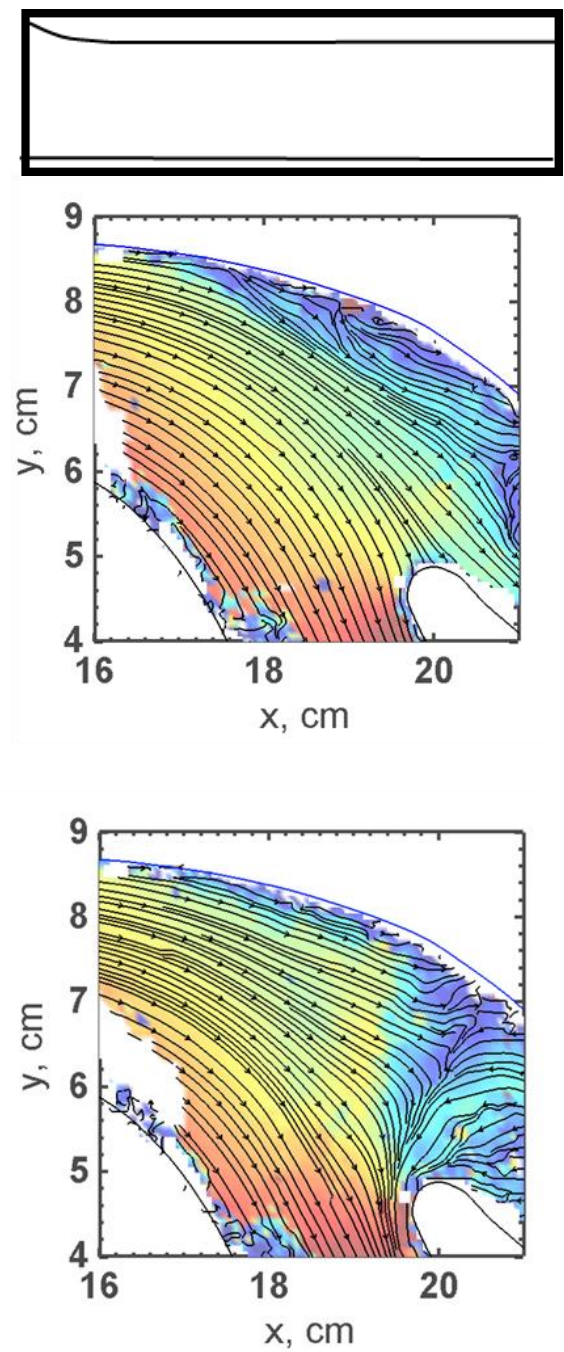
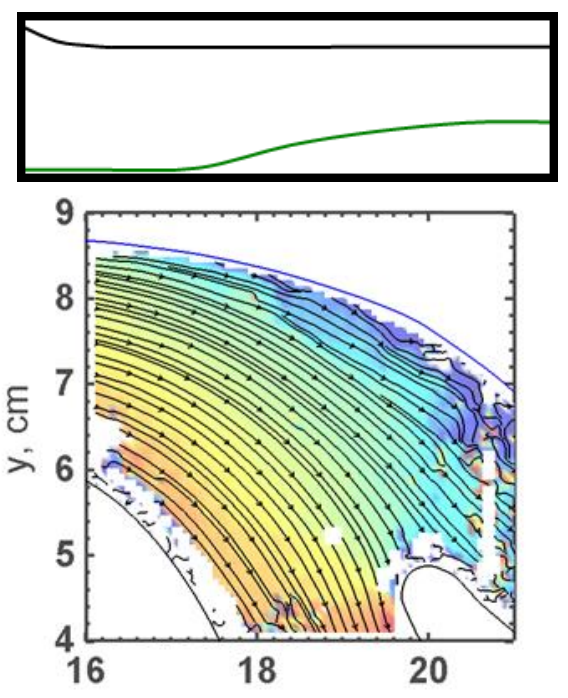

(a)

$\mathrm{X}, \mathrm{cm}$

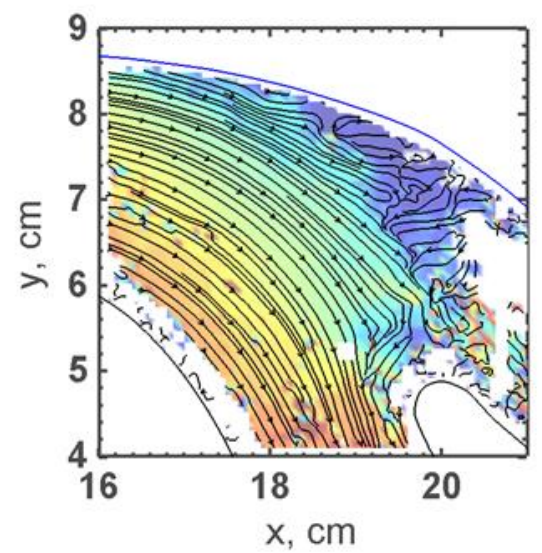

(b)

Figure 1.12. Instantaneous PIV data at $\beta=10 \%$ with the Medium-Hub-Downstream ramp installed. (a) Ideal flow with little separation. (b) Undesirable flow with reingestion from the scavenge leg into the core leg. Actual mass flow rates are $\beta=10.3 \pm 0.1 \%$. 
IPS geometry design is thus to remove the flow unsteadiness completely so that flow-field resembles Fig. 1.12a for as much of the time as possible. This would then be expected to have the highest particle separation efficiency, the lowest operating range and the lowest power requirement for a given $\beta$.

The PIV results also serve to indicate why the hub-side ramps worked better than the outer-side ramps. Figure 1.13 shows the notional flowfield for: (a) No Ramp, (b) OSG-Side-Downstream, and (c) Hub-Side-Upstream. To explain this result, it is critical to note that the flow velocity is maintained at a high value for the portion which enters the core flow, whereas the portion that enters the scavenge leg must reduce in velocity dramatically owing to the reduced mass flow rate without a commensurate reduction in cross-sectional area. This effectively leads to an adverse pressure gradient for the scavenge flow, causing the flow to separate from the outer surface since most of flow is pulled into the core region. In the No Ramp case, the mean PIV results of Fig. 1.11a indicate that the velocity reduction is more exaggerated on the outer surface. This is notionally indicated by separation for the outer surface in Fig. 1.13a, though, in reality, the separation region is understood to be inherently 3-dimensional and varies widely in size with time. While both hub-side and outer-side ramps reduce the cross-sectional area in the scavenge leg, a ramp placed in

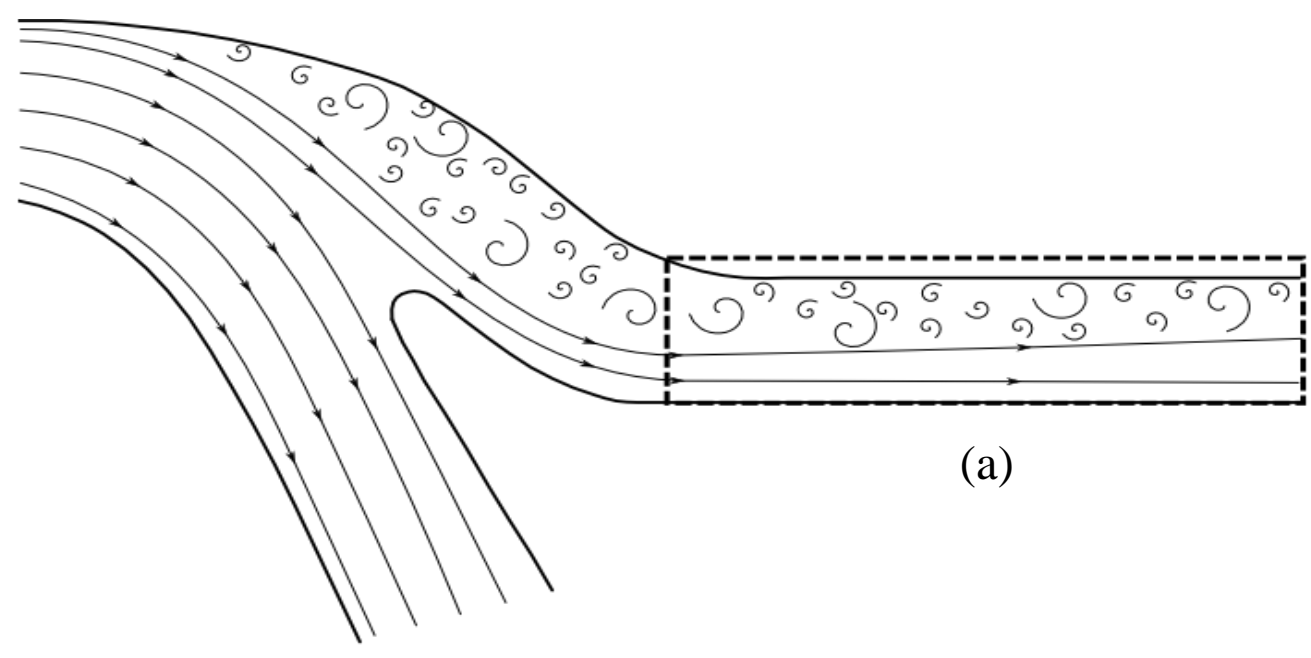

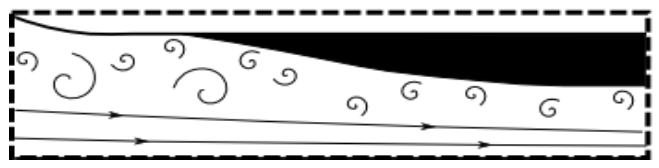

(b)

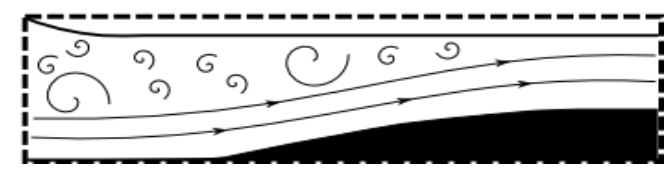

(c)

Figure 1.13. Estimated average flowfields for No Ramp (a), Medium-OSG-Downstream (b), and MediumHub-Downstream (c). Spirals indicate regions with predominantly separated flow. In (a) and (b) separated flow can exit the test section, while in (c) the separation is contained by the attached flow. Particulate caught in separated flow is more likely to be transported upstream and ingested into the core. 
a less separated region would tend to have a greater impact on the flow. This difference is notionally illustrated in Fig. $1.13 \mathrm{~b}$ and $1.13 \mathrm{c}$. As such, if the No Ramp scavenge has show more separation on the hub-side, one might expect a outer-side ramp to allow for greater improvements.

\section{Conclusions}

This study investigated the influence of scavenge flow path geometry immediately downstream of the splitter nose, using a fundamental two-dimensional experimental facility to represent the flow path of an Inertial Particle Separator. The inlet filtration design space often puts the need for maximum separation efficiency in opposition to minimum power requirements. Results of motor power tests indicate that changes to the scavenge leg geometry, specifically medium-Hub ramps, can reduce the total power consumption of the system. In addition, they can allow IPS systems to reach a lower minimum achievable scavenge mass flow fraction ( $\beta$ ). In practice, a lower minimum achievable $\beta$ allows a wider range of possible operating conditions and is associated with improved flow stability. In particular, the Medium-Hub ramp geometry was found to yield significantly better scavenge leg flow stability when compared to the No Ramp condition.

The flow stability improved most with ramps located hub-side (vs. on the outer surface), this suggests that flow separation in this part of the scavenge leg is more likely to occur on the hub-side. The similar benefits (in terms of flow stability) for the longer ramps on either side indicates that this effect may be reasonably localized so that a longer contraction region is not as sensitive to placement. The geometries with the lowest power requirements and best stability also let to the highest particle separation efficiencies.

The PIV results for the mean velocity indicated that the Medium-Hub-Downstream ramp has an increased inflow velocity at the centerline and an increased core flow velocity near the splitter nose when compared to the No Ramp condition, suggesting increased span-wise flow separation for the ramp inclusion. The centerline PIV results also indicated a more spatially-uniform and more stable flow entering the scavenge leg. This increased steadiness may explain the improvement in particle separation efficiency for the inclusion of the ramp. The large central vortical structure is consistent with the scale of a large wall-normal vortex identified by Barone et al. ${ }^{11}$ In that study, large vortices were observed to appear at irregular intervals at very short time scales and caused large amounts of particulate to be reingested into the core. 
The flow visualization and PIV of this study lend credence to the idea that the wall normal vortex and its associated separation instability play a key role in separation efficiency and power consumption. This study showed that a reduction in flow unsteadiness can make improvements to both design goals. Future studies will seek to better characterize and understand the separation and unsteadiness with surface flow visualization and computational methods, among other approaches. The challenge for future IPS geometry design is to remove unsteadiness completely so as to increase separation efficiency to even higher levels with minimum power requirements. 


\section{2: Novel Outer Surface Geometry for an Inertial Particle}

\section{Separator}

\section{Introduction}

For IPS flowpath optimization, a challenging design aspect is the shape of the Outer Surface Geometry (OSG). As detailed in the introduction, IPS geometries must account for large, bounce-dominated particles with high Stokes numbers ( $\mathrm{St}>>1)$ as well as small, drag-dominated particles with low Stokes numbers ( $\mathrm{St}<<1)$. The OSG is critical for both particle regimes, as it is subject to bounces from large particles and can determine the extent of flow separation, which impacts small particles. OSGs must be designed to guide the bouncing particles into the scavenge while keeping flow separation to a minimum.

The present study seeks to interrogate multiphase flowfield changes and particle separation efficiency for a novel OSG, building on the work of Barone. ${ }^{10,11}$ By moving the OSG closer to the hub and decreasing the scavenge channel height, the expansion ratio of the scavenge stream tube may be decreased. This may help to reduce flow separation, and in turn improve particle separation efficiency, ideally without adversely affecting particle bounce profiles. The current study is performed on a University of Virginia fundamental two-dimensional experimental facility which allows detailed optical access and accurate particle separation results.

\section{Methods}

\section{A. Test Facility}

Tests are conducted in a wind tunnel facility at the University of Virginia ${ }^{8,17}$ (Figs. 2.1 and 2.2). The test section is a full-scale version of the flow path elements of an IPS using similar geometry converted from a two-dimensional axisymmetric geometry. By ensuring full-scale flow path dimensions in a span wise cut and full flow velocities, there was no need to employ Reynolds, Mach, or Stokes number scaling, assuming standard sea-level conditions. To eliminate gravitational bias normal to the flow for the particle trajectories, a vertical pull-down wind tunnel is used. Upstream of the wind tunnel entrance, the particles are dispersed to dilute conditions by compressed air ejection, 
which prevents undesirable clumping. The resulting particle cloud simulates high particle loading that can occur during austere helicopter landing conditions.

The particulate then enters the present wind tunnel facility through an inlet with a flow straightener and a smooth contraction into the test section, where it bifurcates into the scavenge and core legs. Diffusers in each leg (scavenge and core) connect the tunnel to air filters, which collect the test dust to allow separation efficiency measurements. Downstream of the filters, the flow in each leg follows a duct system to its respective blower motor and is then exhausted into ambient (outdoor) conditions.

The rig for the present study has been upgraded from previously published results. The diffuser in the core leg (Fig. 2.1b) is now designed to be longer and less aggressive to minimize estimated power losses ${ }^{18}$ and allows a wider range of operating conditions. 20 static pressure taps (five per sidewall) have been added to the diffuser to allow performance monitoring if desired. Procedure updates to the separation efficiency technique can be found in that section below.

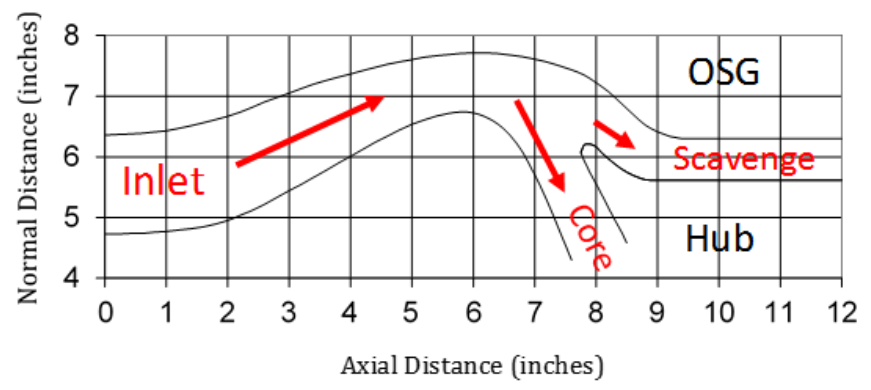

(a)

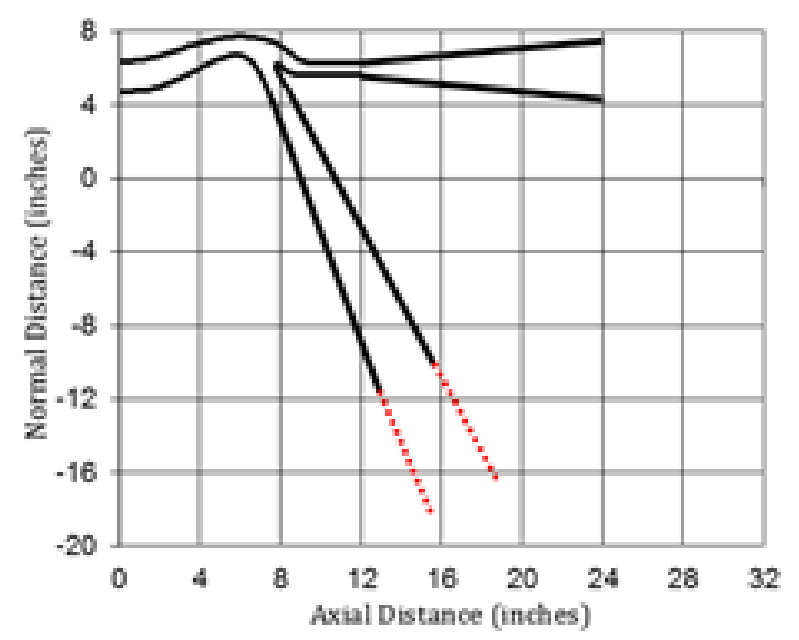

(b)

Figure 2.1. Schematics of an Inertial Particle Separator (IPS). (a) Test section, bounded by Hub geometry and Outer Surface Geometry (OSG). Particulate-laden airflow enters at the inlet, then undergoes a contraction and bifurcation. Particulate is ejected out the scavenge leg while the cleaned air flows through the core leg to the engine. Depth (normal to the page) is a constant 8 inches throughout the test section. (b) Schematic showing diffusers of Connolly ${ }^{6 \mathrm{X}}$ and Barone $^{8}$ with the new extended core diffuser highlighted in red.

\section{B. Mass Flow Control}

The scavenge mass flow fraction $(\beta)$ is defined as the ratio of scavenge leg mass flow rate $\left(\dot{m}_{s}\right)$ compared to the total inlet mass flow rate, which is the sum of the mass flow rates in the core leg $\left(\dot{m}_{c}\right)$ and scavenge leg $\left(\dot{m}_{s}\right)$, i.e.

$$
\beta=\frac{\dot{m}_{S}}{\dot{m}_{S}+\dot{m}_{c}}
$$




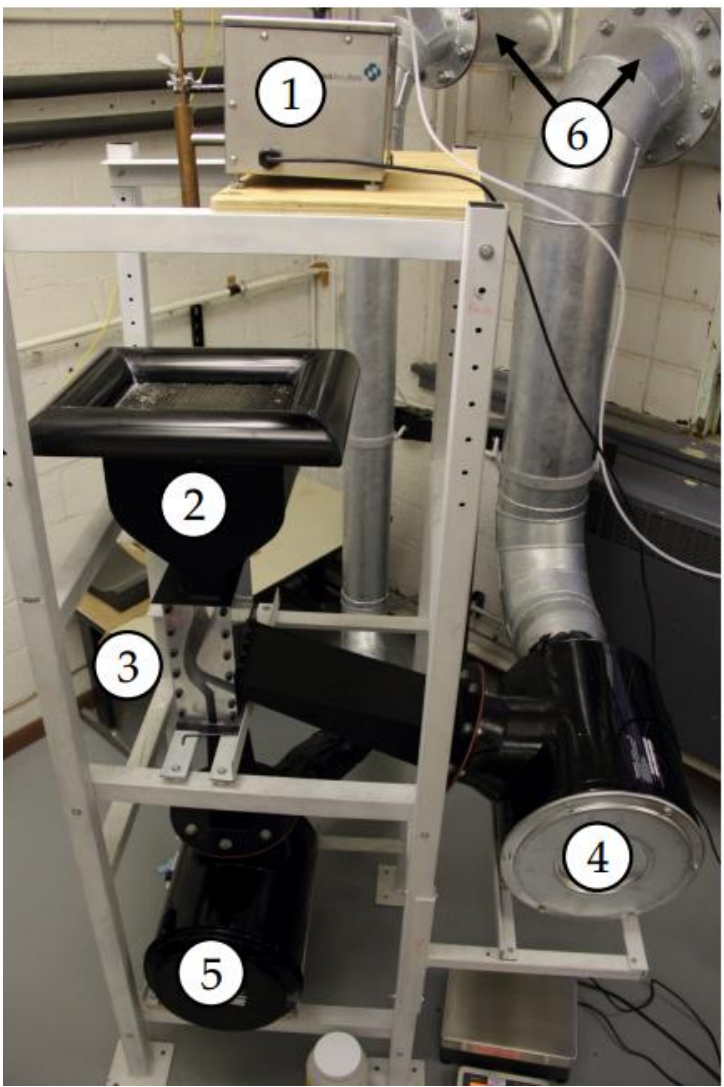

(a)

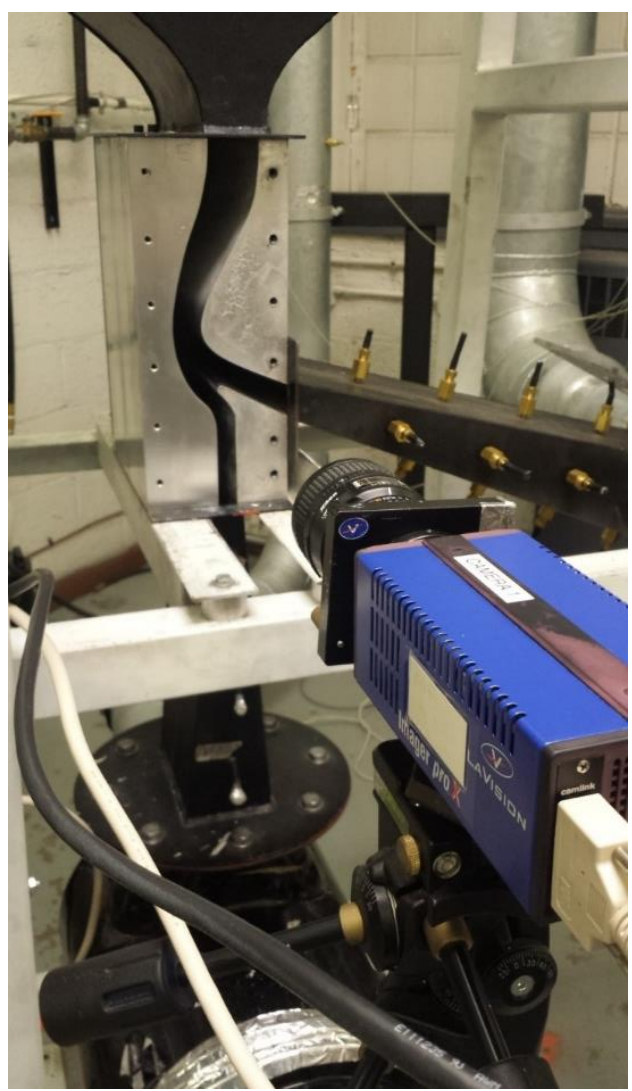

(b)

Figure 2.2. Test rig. (a) Setup for particle separation efficiency test. Particulate is ejected from the Particle Delivery System (1), enters the inlet (2) and test section (3), then flow bifurcates and passes through the core (4) and scavenge (5) filters. Downstream of the filters, the particulate leaves through ducts (6) toward the blowers outside. (b) Setup for Particle Image Velocimetry.

The flow is driven by exhaust fans that are controlled to achieve the proper $\beta$ values. During a typical test, the core volumetric flow rate is held ideally constant at 1365 CFM (Cubic Feet per Minute) or $0.6442 \mathrm{~m}^{3} / \mathrm{s}$, a value that is consistent with equivalent flow rates per unit span of an actual engine. Desired values of $\beta$ are then obtained by varying the scavenge leg mass flow rate.

The density at each flow meter is needed to find the corrected mass flow rate from volumetric flow rate. Three pressure taps at the same duct location as each of the volumetric flow meters send pressure data to a Scanivalve DSA 3217 pressure scanner with a manufacturer rated $0.03 \%$ uncertainty. For each leg, the three pressures are averaged to obtain a local pressure at each of the flow stations (dynamic pressure in the ducts is on the order of $0.1 \%$ of the static pressure and so is not used for density calculation). Atmospheric temperature, pressure, and relative humidity are recorded for each test. To compute air density at each of the measurement stations, the local pressure is combined 
with atmospheric temperature and humidity to obtain a moist air density based on the IUPAC 1981 standard. ${ }^{14}$ It is assumed that temperature and humidity do not vary significantly from the atmospheric values to the flow meters. Mass flow for each leg is then obtained by multiplying the calculated air density at the meter and the measured volumetric flow at the meter. The mass flow in each leg is then corrected to standard conditions to maintain Mach number consistency in the test section for all tests. The correction given by Mattingly ${ }^{15}$ is employed with an additional correction for humidity as follows:

$$
\dot{m}_{\text {corr }}=\frac{\dot{m}_{\text {meter }} \sqrt{\frac{T_{\text {atm }}}{T_{\text {corr }}}}}{\frac{\rho_{\text {atm }, \text { humid }}}{p_{\text {corr }}}} \frac{\rho_{\text {atm,dry }}}{\rho_{\text {atris }}}
$$

The uncertainty of the scavenge mass flow fraction, $\delta \beta$, is conducted per Barone et $_{\text {al. }}{ }^{8}$ :

$$
\delta \beta=\sqrt{\left(\frac{\dot{m}_{s}}{\left(\dot{m}_{s}+\dot{m}_{c}\right)^{2}} \delta \dot{m}_{c}\right)^{2}+\left(\frac{\dot{m}_{s}}{\left(\dot{m}_{s}+\dot{m}_{c}\right)^{2}} \delta \dot{m}_{s}\right)^{2}}
$$

The analysis uses the error in each of the legs, $\delta \dot{m}_{c}$ and $\delta \dot{m}_{s}$, taken as the $2 \%$ reading error reported in the flow meter documented calibration. Note that the uncertainty in the pressure management system is omitted because it is generally two orders of magnitude lower than that of the volumetric flow meters. The values of $\delta \beta$ for all the tests used in this study were about $2 \%$. Full details of the motor controller system may be found in Connolly $e t$ al. ${ }^{17}$

\section{Outer Surface Geometry Design and First Order Methods}

A key factor in the operational performance of an IPS is the design of its Outer Surface Geometry (OSG). Since large particles will impact the OSG, it must be shaped so that their trajectories lead them to the scavenge leg. Additionally, it has a strong effect on the behavior of the flowfield and, particularly, flow separation. This study's new OSG (termed $\mathrm{s} / \mathrm{h}=0.70$ ) as well as the geometries investigated by Barone et $a l .{ }^{10}$ are shown in Figure 2.3. OSGs can be characterized by two key parameters. Throat height $(\mathrm{H})$ is taken here as the distance from the highest point on the hub to the point on the OSG directly above it. $\mathrm{H}$ is constant for all of the OSGs in this study. Scavenge channel height (s) is termed here as the minimum distance from the maximum height of the splitter nose to the nearest point on the OSG. The OSG can then be defined as the ratio of the two parameters, s/H.

This has a physical significance to the fluid physics and separation efficiency of the IPS. Since H $\beta$ is the height of the stream tube at the throat, then $\mathrm{s} /(\mathrm{H} \beta)$ is the expansion ratio of the stream tube. For example, if the scavenge mass flow fraction $\beta=0.10$ then $10 \%$ of the total airflow is entering the scavenge. For a two dimensional flow of constant density approximation, that means that the top $10 \%$ of the throat height $\mathrm{H}$ is part of the scavenge stream tube. 

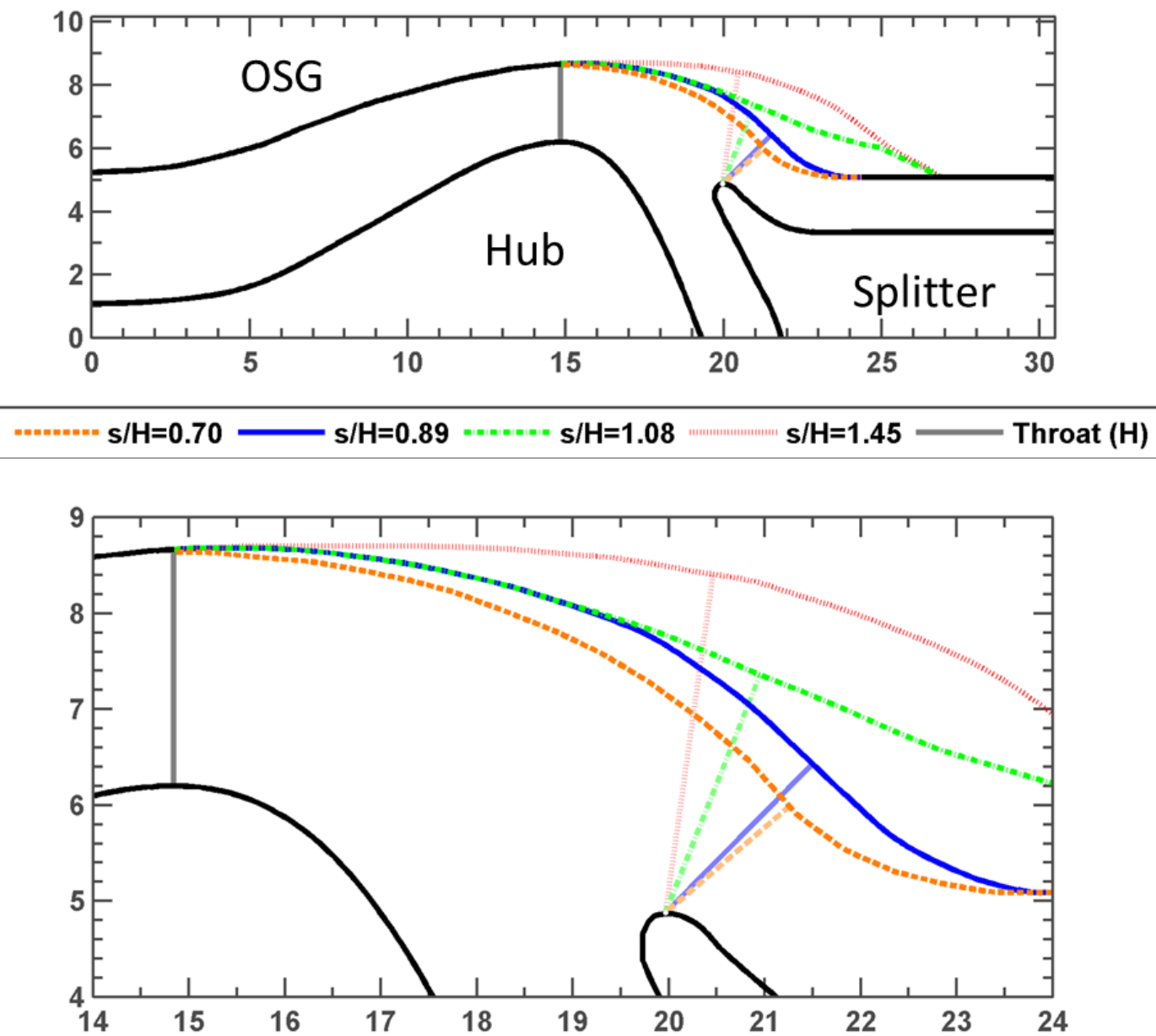

Figure 2.3. Outer Surface Geometries (OSGs). $\mathrm{H}$ is the channel height at the throat, $\mathrm{s}$ is the scavenge channel height measure from the highest point of the splitter nose to the closest wall of the respective Outer Surface Geometry (OSG).

$\mathrm{H} \beta$ is then the scavenge stream tube height at the throat. Further assuming all forward flow in the scavenge leg, $\mathrm{s}$ is then the stream tube height at the scavenge entrance. Expansion ratio of the scavenge streamtube, $s /(H \beta)$, is in this case 7.0. OSGs are classified simply by $\mathrm{s} / \mathrm{H}$ as the value of $\beta$ depends on the operating settings.

Here the problem of optimizing for bounce-dominated particles and drag-dominated particles becomes evident. If $\mathrm{s} / \mathrm{H}$ is large, then the OSG provides a bigger region to "catch" larger bounce-dominated particles. As the expansion ratio increases, however, the possibility of flow separation increases as well. This could cause drag-dominated particles to be reingested into the core engine flow. Connolly ${ }^{17}$ suggests that flow separation as the flow bifurcates may be a primary mechanism of lowered particle separation efficiency. 
Flow separation occurs when the boundary layer detaches from the wall, in this case due to an adverse pressure gradient. A separation point or line has a wall shear of zero, while the upstream flow direction is forward and the downstream flow direction is reversed. That is, the streamlines upstream and downstream of the separation line point toward it. A thorough overview of flow separation structures can be found in Délery. ${ }^{19}$ Prediction of the location of the initial separation point can be computed to varying degrees of accuracy using computational codes. For a priori estimation of separation for design purposes, it can be more convenient to perform a first order analysis. A method provided by $\mathrm{Cebeci}^{20}$ to estimate separation point from just an inviscid flowfield of the geometry was used in this case to inform ISG design. An inviscid flowfield was calculated using ANSYS Fluent for each OSG. Since the no-slip condition cannot be enforced in an inviscid flow, the velocity values along the OSG wall were collected. The coefficient of pressure $\left(C_{p}\right)$ is defined as:

$$
C_{p}=1-\left(\frac{u}{u_{o}}\right)^{2}
$$

where $u$ is the velocity along the wall and $u_{o}$ is the velocity at the onset of the adverse pressure gradient. For this estimation (though not necessarily for the actual flowfield), the adverse pressure gradient is taken to begin at an axial distance of 6 " or $15.24 \mathrm{~cm}$, at the throat. After this point, the channel begins to expand as the flow turns toward the hub side. Cebeci uses Stratford's criteria to predict separation, which is given as:

$$
C_{p}^{1 / 2} x \frac{d C_{p}}{d x}>0.102
$$

This means that separation is predicted when the LHS first reaches a value greater than 0.102 . Note that this method is not intended to make predictions after flow separation occurs. Also, due to the square root term, the method does not make predictions upstream of the onset of separation where $C_{p}$ may be negative. In this case, a first order central difference scheme was used to calculate $d C_{p} / d x$ from the $C_{p}$ values.

\section{Surface Flow Visualization}

Surface flow visualization is used to characterize the large scale flow structures and separation ${ }^{16}$. The tests were conducted with titanium dioxide as the colorant, kerosene as the carrier fluid, and oleic acid as a surfactant. The mixture was painted on the surface of interest just before operation and the flow then causes the suspension to streak, leaving a time-averaged wall shear pattern as the kerosene slowly dries. Note that the carrier fluid for such visualization is typically oil and not kerosene, but due to the highly unsteady nature of flow inside the IPS a more volatile fluid was chosen. Kerosene decreases the drying time significantly, allowing the resolution of more transient 
flow structures often present in separated flows. More details are provided in Connolly et al. ${ }^{17}$ The photographs of the surface flow results have their white balance and contrast adjusted to improve visibility.

\section{E. Separation Efficiency Technique}

To directly measure the particle separation efficiency (by mass) of a given flowpath geometry, approximately $100 \mathrm{~g}$ of test dust is released into the inlet over five minutes of wind tunnel operation by a Particle Delivery System (PDS) volumetric feeder. Once through the test section, the dust is captured in the downstream filters. These filters, as well as the PDS, are weighed before and after each test for determination of the total mass of particulate used in the test. The separation efficiency $(\eta)$ is then calculated as the ratio of the mass gained in the scavenge filter $\left(\Delta m_{s f}\right)$ to the total mass gained by both the core filter $\left(\Delta m_{c f}\right)$ and the scavenge filter:

$$
\eta=\frac{\Delta m_{s f}}{\Delta m_{c f}+\Delta m_{s f}}
$$

For example, in the case where $90 \mathrm{~g}$ of the particulate is captured by the scavenge filter and $10 \mathrm{~g}$ by the core filter, 90\% efficiency is achieved. Mass discrepancies $\left(\delta m_{r}\right)$ can occur if particulate sticks to the sidewalls of the tunnel or passes through the filters. These are recorded for each test by comparing the mass change of the PDS ( $\Delta m_{P D S}$, net particles delivered) to the total mass change of the filters $\left(\Delta m_{c f}+\Delta m_{s f}\right.$, net particles collected):

$$
\delta m_{r}=\Delta m_{P D S}-\left(\Delta m_{c f}+\Delta m_{s f}\right)
$$

All efficiency test data in this report have less than $2 \%$ mass discrepancy.

The full procedure for measuring particulate separation efficiency is detailed in full by Connolly et al. ${ }^{17}$, with the following minor additions. Preventing clumping of dispersed particulate is of special concern when trying to accurately measure separation efficiency. This is because a group of clumped particles will have very different aerodynamic characteristics compared to the same particles when dispersed. In response, all particulate used in this study was unsealed from its container and heated in an oven to between $130 \mathrm{~F}$ and $150 \mathrm{~F}(54 \mathrm{C}$ and $66 \mathrm{C})$ for at least thirty minutes before testing. This aimed to reduce any moisture content that may have been present. Additionally, the

PDS was cleaned out thoroughly before every test to ensure consistent operating conditions. Previous work ${ }^{10,17}$ have not shown any visible signs of particulate clumping, but the potential for high atmospheric humidity and the sensitivities of working with finer particulate in the future prompted these preemptive additions to the test procedure. 
The data for OSGs with s/H of $0.89,1.08$, and 1.45 are from Barone et al. ${ }^{10}$ using those mass flow control and separation efficiency procedures. Tests on $\mathrm{s} / \mathrm{H}=0.89$ using both procedures found negligible $($ mean $<1 \%)$ differences in separation efficiency $(\eta)$ for A4 particulate. The procedural differences arise from attempts to simplify operating assumptions, account for a wider variety of atmospheric conditions, and improve test repeatability.

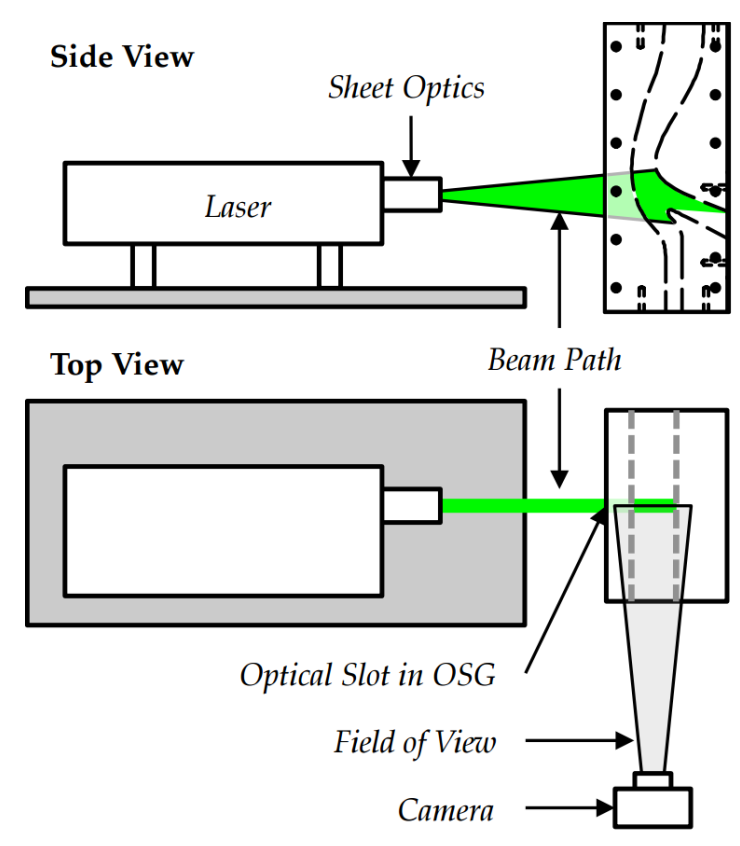

Figure 2.4. PIV setup schematic

\section{F. PIV Technique}

Particle Image Velocimetry (PIV) is a method commonly used in gas flows to quantify the velocity field. It employs tracer particles seeded into the flow that are small enough in size so that they faithfully follow the surrounding airflow. The experimental approach used herein utilizes olive oil droplets of approximately $1 \mu \mathrm{m}$ diameter, because they very closely follow the flow due to their low Stokes number. These particles are illuminated with a laser sheet, and tracked using a camera (Fig. 2.4). The flow's velocity field is calculated using a pair of captured images that contain the same set of particles after they have travelled a short distance. The field is subdivided

into a discrete grid of sub-regions referred to as interrogation spots to obtain a two-dimensional matrix of velocity vectors. For each of these sub-regions, the particles that have traversed a small distance across two successive images are cross-correlated. The distance vector from the cross-correlation is divided by the time between the two images resulting in the velocity vector. By taking a large number of these image pairs throughout an experiment, an average flow field can be determined, along with the statistical variations present. Notably, the particle species fraction in the air is $<0.02 \%$, so no difference is anticipated between the particulate laden flowfield, the olive oil laden flowfield, or the unladed flowfield.

In the present set-up, the laser sheet is positioned at the centerline of the test section width so as to provide the clearest view of the flow at the bifurcation in order to characterize the flow in the region associated with the highest amounts of instability. The PIV camera is used to capture approximately 1000 image pairs, which are interrogated to examine instantaneous flow structures and velocity vectors. The camera records image pairs at a rate of $7 \mathrm{~Hz}$, which 


\begin{tabular}{ccccccc}
$\mathrm{s} / \mathrm{H}$ & Seed & $\beta$ & $\mathrm{T}$ & $R H$ & $p$ & $\mathrm{~N}$ \\
\hline 0.70 & Olive Oil & 0.160 & $69.8 \mathrm{~F}$ & $38 \%$ & $29.1 \mathrm{inHg}$ & 1000 \\
0.89 & Olive Oil & 0.160 & $75.0 \mathrm{~F}$ & $45 \%$ & $29.9 \mathrm{inHg}$ & 2000 \\
1.08 & Olive Oil & 0.160 & $68.3 \mathrm{~F}$ & $54 \%$ & $29.7 \mathrm{inHg}$ & 1000 \\
1.45 & Olive Oil & 0.160 & $65.2 \mathrm{~F}$ & $34 \%$ & $30.2 \mathrm{inHg}$ & 1000
\end{tabular}

Table 2.1. Test Conditions for Gas PIV. T is temperature, RH is relative humidity, $p$ is atmospheric pressure, and $\mathrm{N}$ is the number of image pairs taken.

is sufficient for capturing instantaneous flow velocities and structures. This data was averaged to obtain the mean velocity and standard deviation of the velocity of the flow. This allows gas velocity to be measured within $2 \%$ accuracy. ${ }^{11}$ Atmospheric conditions were recorded before each experiment (Table 2.1). Scavenge mass flow fraction was set to $\beta=0.16$ to maintain consistency with previous results.

This study used a slightly different PIV system than the previous studies. ${ }^{11,12}$ An LaVision Imager Pro X camera (Fig. 2.2b) was used to take the image pairs, and $\mathrm{DaVis} 7.2$ was used to process the data. The time between image pairs was $5 \mu \mathrm{s}$ rather than the $2 \mu \mathrm{s}$ used previously. For an interrogation region of $1 \mathrm{~mm}$ and flow at $200 \mathrm{~m} / \mathrm{s}, 5 \mu \mathrm{s}$ is the approximate time for the flow to traverse the region, and therefore the maximum $\Delta \mathrm{t}$ for the PIV system. Previously, $2 \mu \mathrm{s}$ was used to further reduce uncertainty, but the $5 \mu$ s time window of the current study does not violate the operating assumptions.

The camera is limited to an acquisition rate of $10 \mathrm{~Hz}$, while the instantaneous flow structures appear on the order of $1000 \mathrm{~Hz}$. Therefore a significantly more advanced system is necessary to capture time-resolved PIV. Other methods like high speed video can be employed for additional transient flowfield characterization. ${ }^{11}$

\section{Results}

Investigations of a novel outer surface geometry were performed on a full-scale Inertial Particle Separator test rig. Surface flow visualization, first order separation prediction, A4 particle separation efficiency, and PIV experiments 


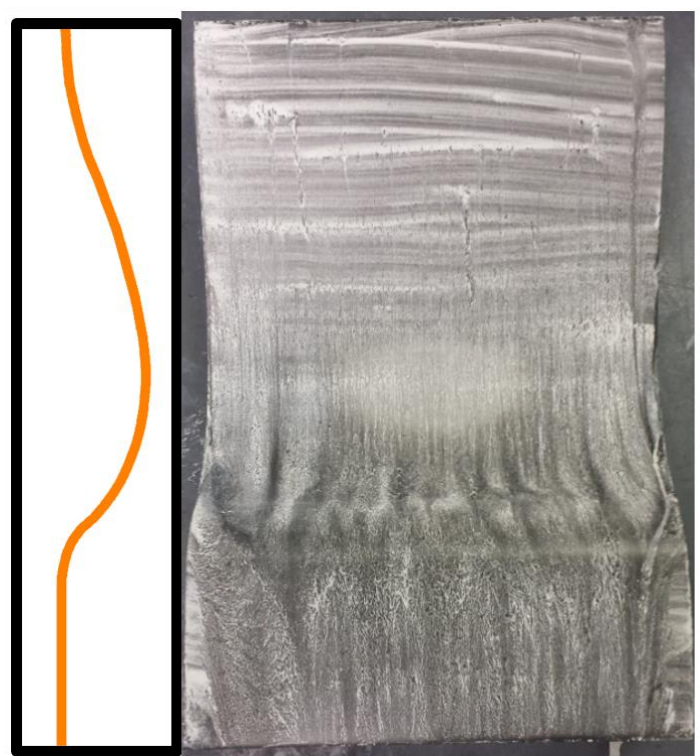

Figure 2.5a. Surface Flow Visualization of $\mathbf{s} / \mathbf{H}=\mathbf{0 . 7 0}$. Note that the horizontal lines in the upstream portion of the flow are caused by the brush applying the mixture to the surface and are not representative of actual flow features 1

\section{A. Surface Flow Visualization Results}

The result of the surface flow visualization Flow for s/H $=0.70$ (Fig. 2.5a) shows clearly defined flow separation. A moderate saw tooth separation pattern can be observed. Sidewall separation begins just upstream of the main flowpath separation and extends to 1-2" from the wall in the scavenge channel. Horizontal lines in the upstream portions of the flow are due to the mixture application procedure and are not reflective of actual flow features. Compared to the other OSGs (Fig 2.5b, Table 2.2), s/H=0.70

Flow
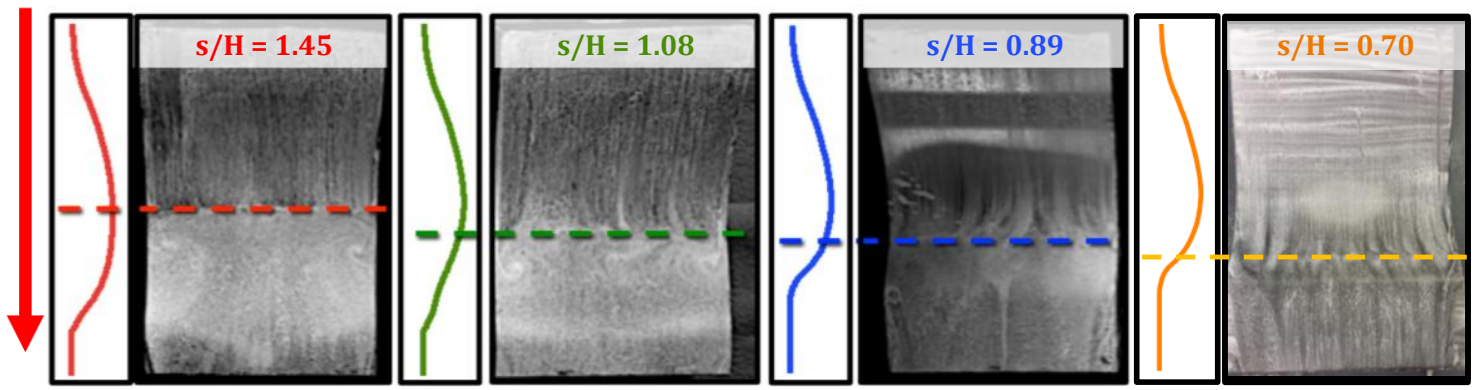

Figure 2.5b. Surface Flow Visualization Comparison

\begin{tabular}{ccccc}
$\mathrm{s} / \mathrm{H}$ & $\eta$ at $\beta=0.16$ & $x_{\text {start }}$ & $x_{\text {end }}$ & $\mathrm{L}$ \\
\hline 0.70 & 0.91 & 19.9 & 23.1 & $3.2 \mathrm{~cm}$ \\
0.89 & 0.90 & 19.2 & 23.2 & $4.0 \mathrm{~cm}$ \\
1.08 & 0.89 & 18.9 & 26.0 & $7.1 \mathrm{~cm}$ \\
1.45 & 0.85 & 16.5 & 26.8 & $10.3 \mathrm{~cm}$
\end{tabular}

Table 2.2. Surface Flow Visualization results. All tests conducted at the same scavenge mass flow fraction $\beta$. $\eta$ is the average particle separation efficiency at that condition. $x_{\text {start }}$ and $x_{\text {end }}$ denote the boundaries of the observed flow separation region, while $\mathrm{L}$ is the total length of the region 
has the furthest downstream separation. There seems to be a lack of large scale wall-normal vortical structures as well. Comparing the results of all four surface flow visualizations, there is clearly a direct correlation between s/H and separation region length $(\mathrm{L})$, as well as the approximate separation $\left(\mathrm{x}_{\text {start }}\right)$ and reattachment $\left(\mathrm{x}_{\text {end }}\right)$ points. The new $\mathrm{s} / \mathrm{H}=0.70$ has the shortest separation region, latest separation location, and earliest reattachment location of all OSGs, in addition to the highest separation efficiency (discussed below).

\section{B. Stratford's Criteria}

The results of the simple inviscid flowfield (Fig. 2.6) show lower pressures and increased velocities for $\mathrm{s} / \mathrm{H}=$ 0.70. The simulations are not meant to be representative of the actual flowfield inside the IPS, but provide the necessary data to predict flow separation using Stratford's criteria. The coefficient of pressure $\left(\mathrm{C}_{\mathrm{p}}\right)$ and its gradient are computed along the OSG wall from the inviscid solutions (Fig. 2.7a). The estimated location of the onset of the adverse pressure gradient is $6 "(15.24 \mathrm{~cm})$ in the axial direction, near the throat. The area of interest for separation is marked with vertical red lines.

The result of the calculation of Stratford's Criteria for each OSG is shown in Figure 2.7b (zoomed to the area of interest). The predicted separation points are upstream from those shown by the surface flow visualization. It shows $\mathrm{s} / \mathrm{H}=1.45$ separating first, with $\mathrm{s} / \mathrm{h}=1.08$ and 0.89 nearly identical, and $\mathrm{s} / \mathrm{H}=0.70$ separating last. Note that $\mathrm{s} / \mathrm{h}=$ 1.08 and 0.89 share identical upstream geometry profiles until $7.6 "(19.5 \mathrm{~cm})$ in the axial direction. Stratford's criteria relies on inviscid models and fails to take into account downstream conditions, though the flow visualization indicates that the separation points of both geometries are very close to each other. From a qualitative perspective Stratford's method is an easy way of seeing if a new flowpath design is likely to delay separation. It is an effective way of trialing potentially large number of geometries with negligible computational cost, in advance of more rigorous computational or experimental investigations. Such a method could be combined with a bounce model to account for both drag- and bounce-dominated particles. 


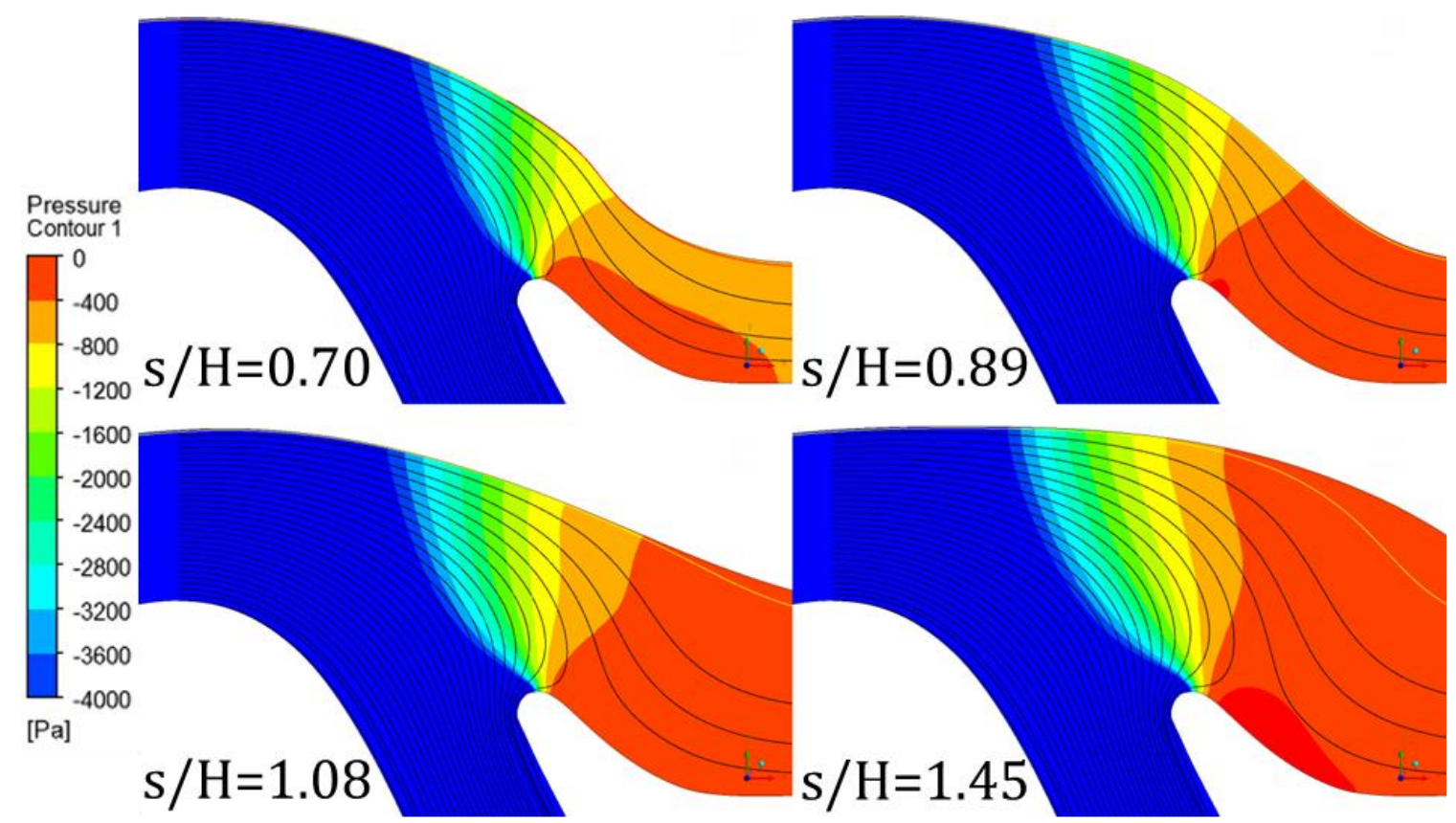

(a)

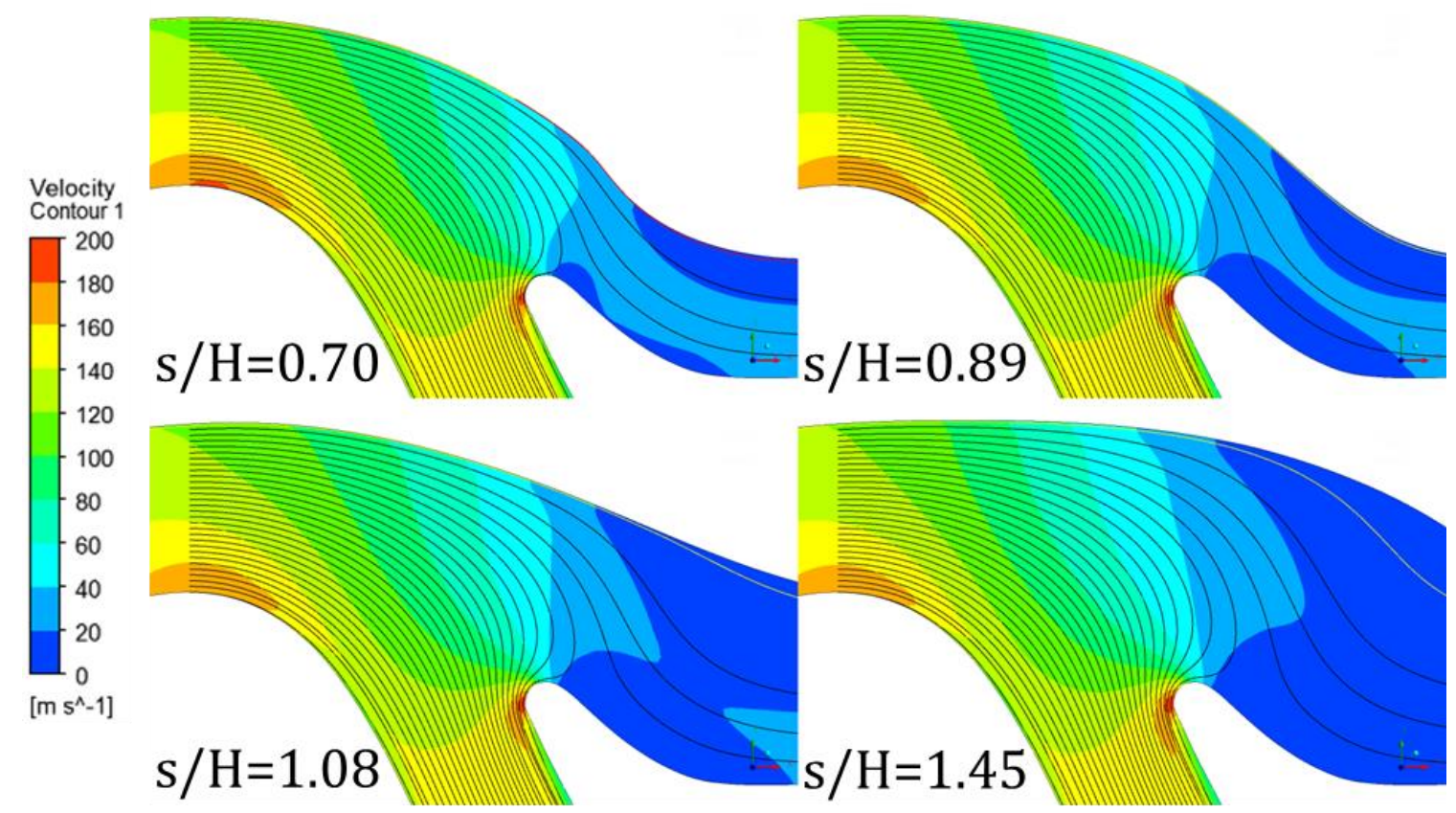

(b)

Figure 2.6. Inviscid CFD Pressure (a) and Velocity Contours (b) at $\beta=10 \%$. 


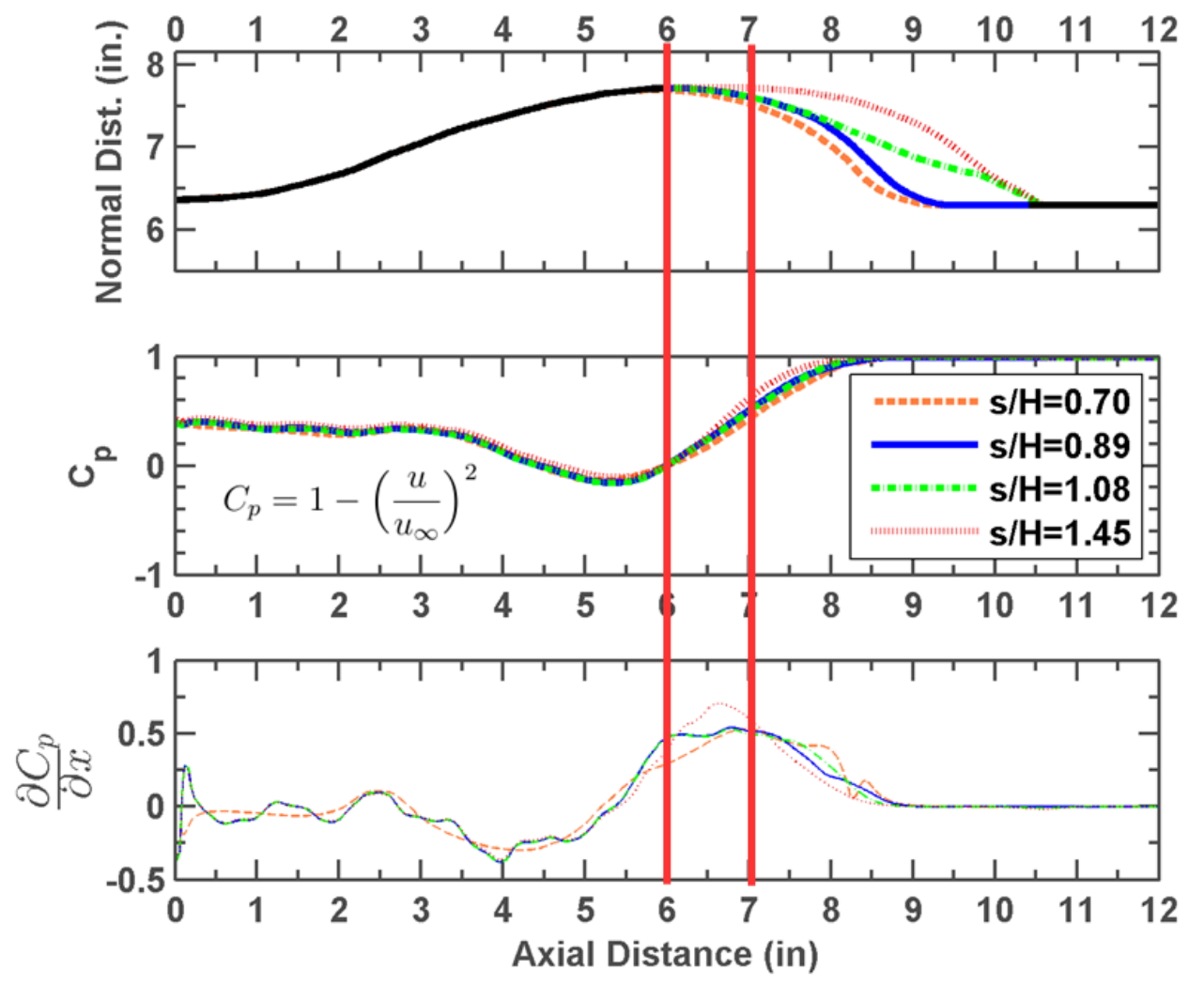

Figure 2.7a. Statistics from Inviscid Flow Solution.

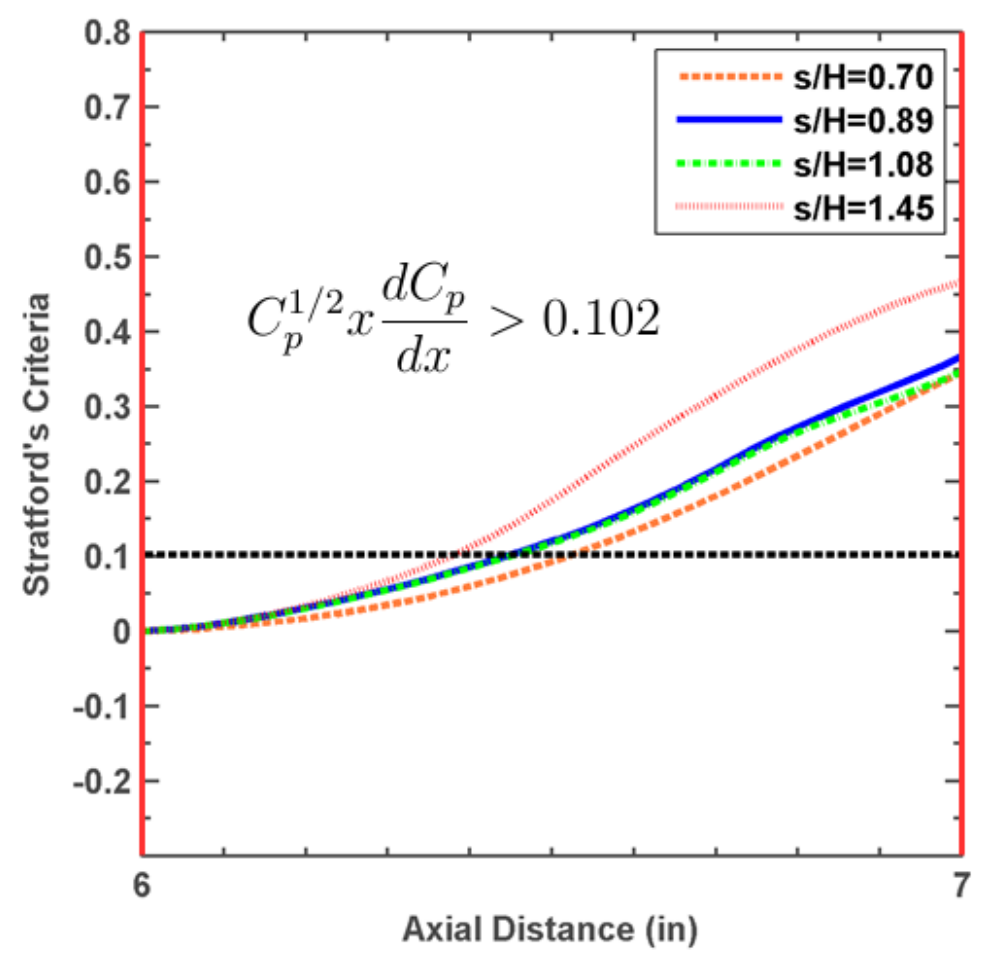

Figure 2.7b. Stratford's Criteria for Separation Prediction 


\section{PIV Results}

Time-averaged PIV velocity and flow standard deviation are shown for each OSG (Fig. 2.8). The velocity field for $\mathrm{s} / \mathrm{H}=0.70$ is similar to that of $\mathrm{s} / \mathrm{H}=0.89$, with perhaps slightly delayed separation. Core flowfields are very similar for each OSG. In the scavenge region, the standard deviation of the velocity decreases with s/H. Note a large, fully developed separated flow and high standard deviation in the scavenge leg of $\mathrm{s} / \mathrm{H}=1.45$. As $\mathrm{s} / \mathrm{H}$ decreases, the mean recirculation region shrinks $(\mathrm{s} / \mathrm{H}=1.08)$ and disappears $(\mathrm{s} / \mathrm{H}=0.89$ and $\mathrm{s} / \mathrm{H}=0.70)$. Additionally, $\mathrm{s} / \mathrm{H}=0.70$ has a smaller region of low speed flow in the scavenge leg, indicating potentially reduced separation. That is not to say that recirculation or separation is eliminated for low $\mathrm{s} / \mathrm{H}$, but its incidence has reduced to the point that the mean flow is forward.

For $\mathrm{s} / \mathrm{H}=0.70$ the region near the hub wall has high standard deviation. These may be attributed to laser reflections along that wall. The camera was aligned to capture the flow in the scavenge leg and to minimize laser reflections there. As a consequence, laser reflections on the hub caused some noise. Based on the other observation methods of this study, it seems highly unlikely that the core flow for $\mathrm{s} / \mathrm{H}=0.70$ is more unsteady than for any of the other geometries.

Instantaneous flowfields (Fig. 2.9) show a few of the flow conditions observed by the PIV camera in lieu of displaying all 1000 velocity fields. Separation in the PIV frames is observed to vary from frame to frame near the point suggested by the surface flow visualization. Typical flow may be characterized by the left four images, with mild separation and a steady flow stream to the scavenge. Large scale separation and core reingestion (top right) is unfortunately also present occasionally. Since $\beta$ is high, reingestion events are generally uncommon. Any small, dragdominated particles $(\mathrm{St}<1)$ caught in the scavenge during this flow event have a high likelihood of being reingested into the core. ${ }^{10}$ On the other hand, instances of negligible flow separation (bottom right) show what an ideal IPS flowfield might look like. 

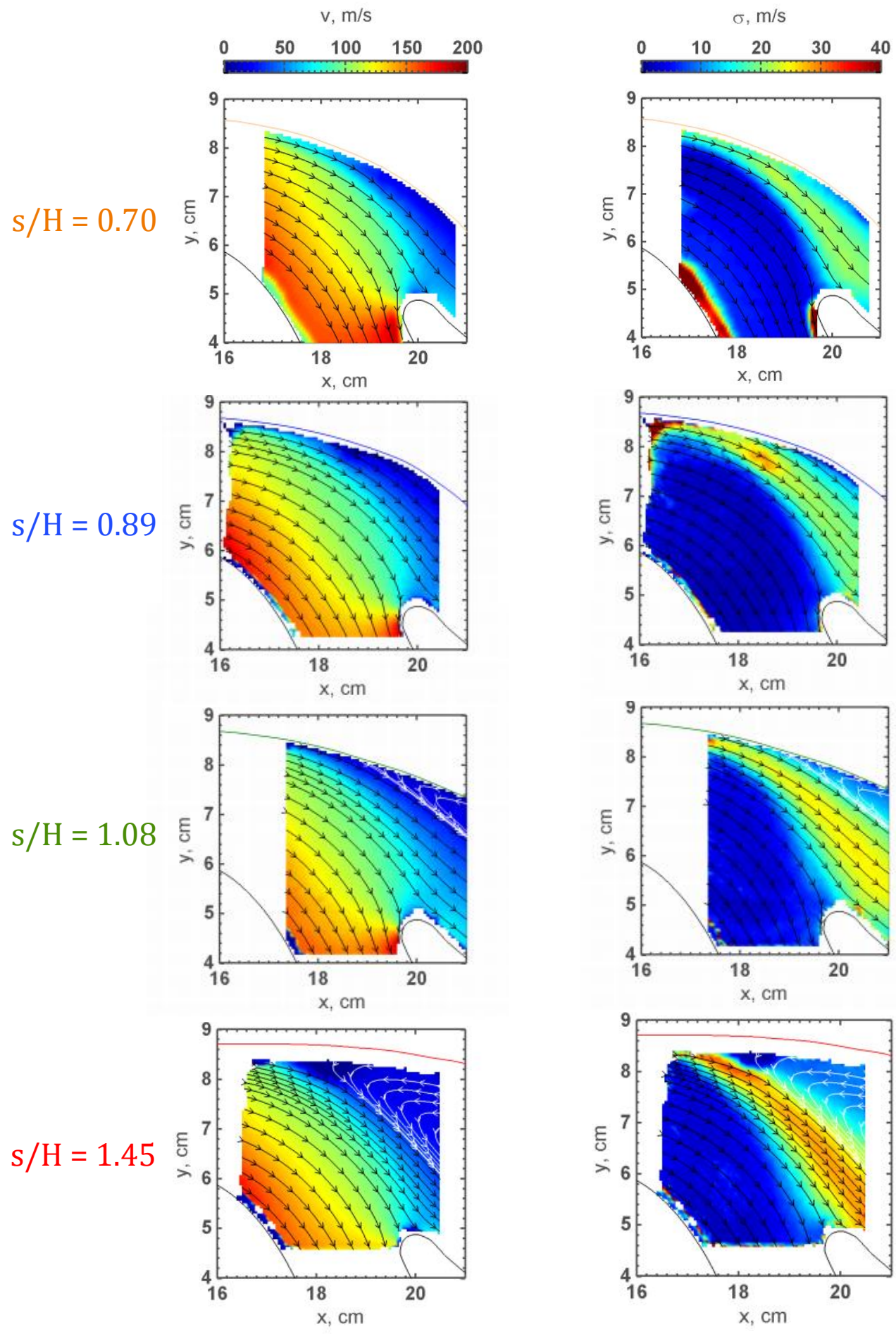

Figure 2.8. Time Averaged PIV at $\beta=0.16$ 

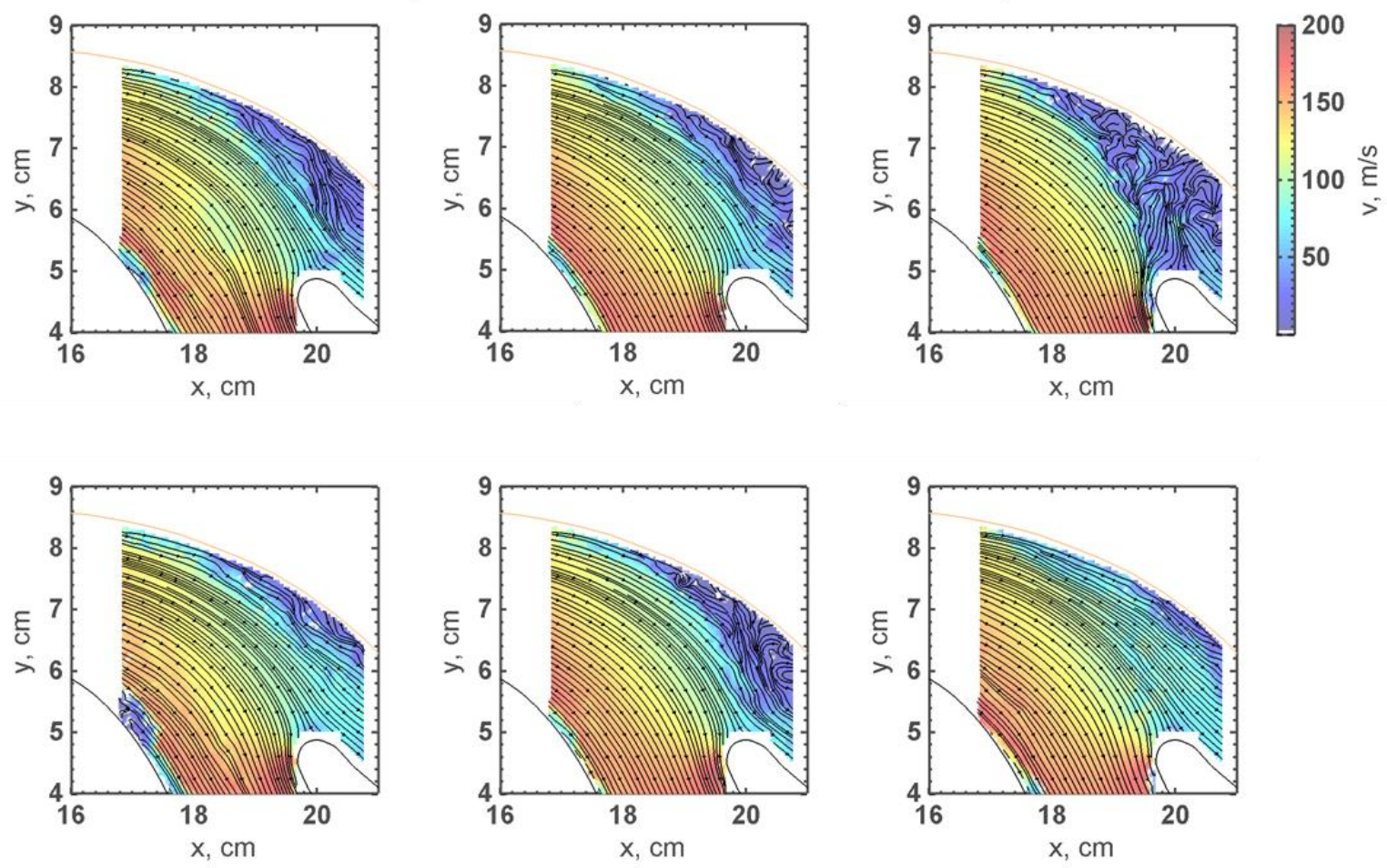

Figure 2.9. Instantaneous $\mathrm{PIV}$ of $\mathrm{s} / \mathrm{H}=0.70$ at $\beta=0.16$

To more quantitatively compare OSG cases a velocity rake of the scavenge (Fig. 2.10) is taken at an axial distance of 7.9" (20 cm). This corresponds with the highest point on the splitter nose where s was defined (Fig. 2.3) and the entrance to the scavenge channel. The mean flows in the axial direction (shown in the contour plots of Fig. 2.8) are solid lines, while the dashed lines represent \pm 1 standard deviation $(\sigma)$ in axial flow velocity. As can be inferred from the instantaneous PIV frames, there is quite a bit of variation in the scavenge velocity. Compared to the other OSGs, $\mathrm{s} / \mathrm{H}=0.70$ saw almost no flow reversal within one standard deviation. Theoretically, if flow in the entire scavenge was strictly forward at all times, there could be no unwanted reingestion events. By this criteria, $\mathrm{s} / \mathrm{H}=0.70$ is an improvement over the other geometries. 

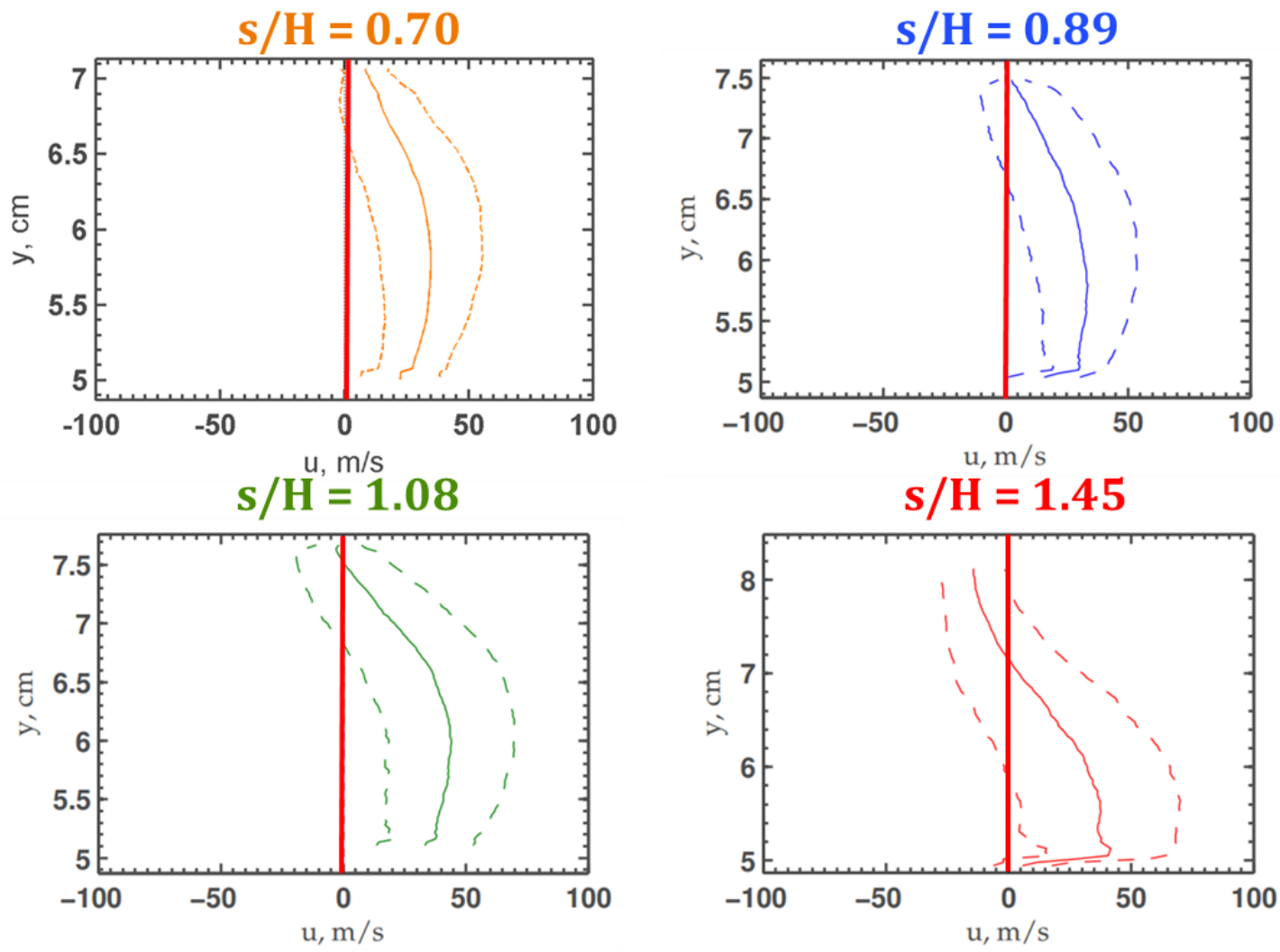

Figure 2.10. PIV Scavenge time averaged velocity rakes. Solid lines are mean flow, dashed lines are the mean $\pm 1 \sigma$. Note different scales in the y direction due to varied scavenge channel heights.

\section{Separation Efficiency Results}

A4 particle separation results for $\mathrm{s} / \mathrm{H}=0.70$ are plotted against the results of Barone et $\mathrm{l}^{10}$ for a range of scavenge mass flow fraction, $\beta$ (Fig. 2.11). Each diamond symbol of the new data is the mean of two tests at that flow condition. None of the tests at each $\beta$ varied by more than a percent. For high $\beta(0.16$ and 0.20$)$ the new geometry has the highest particle separation efficiency, $\eta$, by $0.5 \%$ to $1 \%$. The performance is very similar to the other OSGs except for $\mathrm{s} / \mathrm{H}=$ 1.45. Flow separation in this region is known to be present but limited, and reingestion events are relatively rare.

For $\beta=0.10$ and 0.09 , near the lower edge of IPS operating capabilities, $\mathrm{s} / \mathrm{H}=0.70$ outperforms the previous best OSG, $\mathrm{s} / \mathrm{H}=0.89$. There is still a drop off in efficiency for low $\beta$, but it seems to be slightly delayed for the new geometry. This is a significant result, as $\beta$ is directly related to the power costs of the system and increased separation efficiency would allow the system to operate at lower $\beta$. Additionally, it further supports the idea that reducing flow separation can increase separation efficiency for drag-dominated particles. 


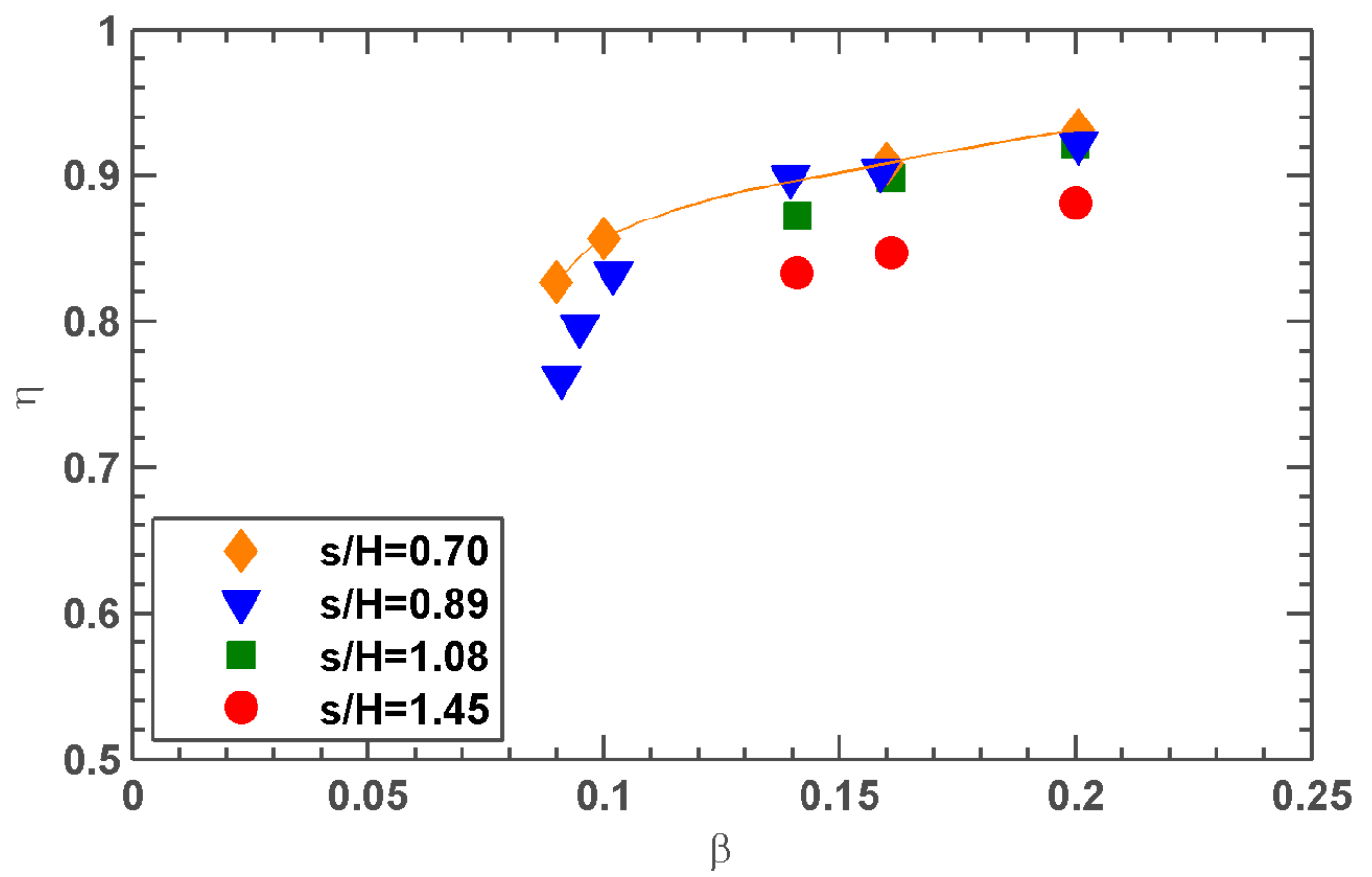

Figure 2.11. Particle Separation Efficiency results. $\mathrm{s} / \mathrm{H}=0.70$ has a cubic interpolation fit line.

\section{Conclusions}

Investigations of a novel Outer Surface Geometry (OSG) for an Inertial Particle Separator were conducted at a fundamental two-dimensional experimental facility. Surface flow visualization, first order methods, Particle Image Velocimetry (PIV), and particle separation efficiency experiments were conducted to characterize the fluid flowfield in the new geometry, as well as evaluate its operational performance. The new geometry delayed flow separation, reduced unsteadiness in the scavenge leg, and improved particle separation efficiency compared to previous geometries.

Surface flow visualizations showed a dependence between $\mathrm{s} / \mathrm{H}$ and flow separation severity. Lowering s/H resulted in delayed flow separation over a shorter separation region. First order methods used to predict the separation point provided a qualitative estimate that may be useful for rapidly developing new geometries at negligible computational cost. 
The new OSG was successful in increasing particle separation efficiency, particularly at low $\beta$. The line connecting the $\mathrm{s} / \mathrm{H}=0.70$ data points (Fig. 2.11) is a cubic interpolation that forms an estimated Pareto front for the design space. More data points in the range $0.10<\beta<0.16$ would provide a more accurate view, but currently it is sufficient to see that separation efficiency increases with decreasing $\mathrm{s} / \mathrm{H}$. Clearly there is a limit to these benefits, since if $\mathrm{s} / \mathrm{H}=0$ there would be no scavenge flowpath. A more immediate constraint is the need to accommodate bouncedominated particles. Further decreasing the s/H risks causing large particles to bounce back into the core. The A4 test particulate used in this study is composed of large $(\mathrm{St}>1)$ and small $(\mathrm{St}<1)$ particulate, but additional study for very large particles $(\mathrm{St}>>1)$ would be helpful in better characterizing the bounce effects this and future geometries.

The newly defined parameter s/H provides a convenient way to quickly asses the scavenge streamtube expansion ratio and therefore the potential for flow separation. Flow separation and particle separation efficiency were found to vary with $\mathrm{s} / \mathrm{H}$ at a constant $\beta$. Particle separation efficiency varied with $\mathrm{s} /(\beta \mathrm{H})$ for a range of $\beta$. It was known previously that both $\beta$ and geometry played an important role in separation efficiency, but this is the first study to the author's knowledge to quantify the relationship between the two in terms of a scavenge streamtube expansion ratio parameter. Accurate predictive functions to describe these relationships for a range of geometries and flow conditions could simplify the IPS design space and guide future development. 


\section{3: Parametric Study of Scavenge Channel Height in an}

\section{Inertial Particle Separator}

\section{Introduction}

Scavenge leg geometry can play a critical role in IPS flowfield stability, as observed in Connolly et al. ${ }^{17}$ and expanded upon in Chapter 1. Reductions in scavenge channel height, when made from the hub, were found to improve particle separation efficiency and reduce system power requirements. Those promising (and surprising) results spurred the development of additional scavenge leg contractions to see if the successes could be improved upon.

A new terminology is adopted for the new geometries to simplify reporting. The Medium-Hub-Downstream case of the previous study is now simplified to Inner Surface Geometry 1, or ISG-1. The two new designs, which create more aggressive contractions, are designated ISG-2 and ISG-3. The changes result in a narrower scavenge channel, which is intended to function more efficiently at lower system power requirements.

The present study seeks to interrogate multiphase flowfield changes and improve particle separation efficiency using these novel scavenge leg geometries. Additionally, this will be the first IPS study to include AFRL 03 test particulate, to the author's knowledge. This smaller particulate is more difficult to separate than A4, and more subject to unsteady flowfields. The current study uses the University of Virginia fundamental two-dimensional experimental facility which allows detailed optical access and accurate particle separation results.

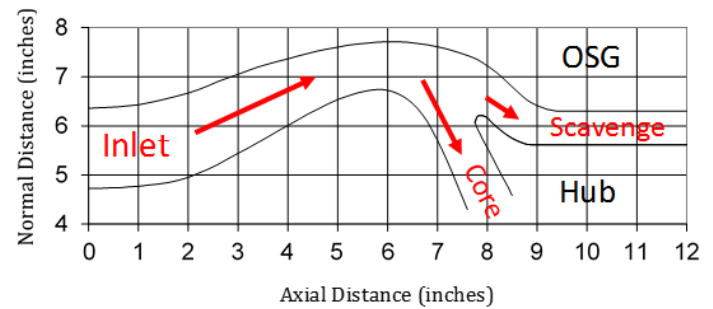

(a)

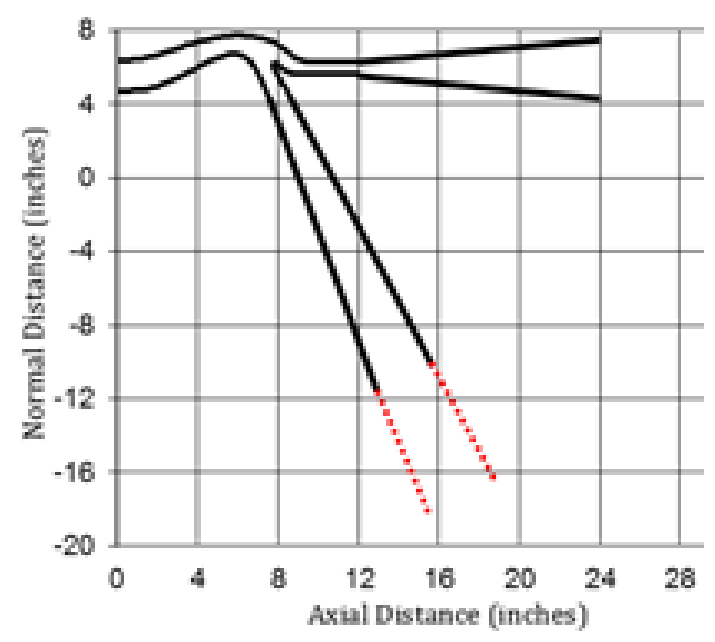

(b)

Figure 3.1. Schematics of an Inertial Particle Separator (IPS). (a) Test section, bounded by Hub geometry and Outer Surface Geometry (OSG). Particulate-laden airflow enters at the inlet, then undergoes a contraction and bifurcation. Particulate is ejected out the scavenge leg while the cleaned air flows through the core leg to the engine. Depth (normal to the page) is a constant 8 inches throughout the test section. (b) Schematic showing diffusers of Connolly ${ }^{6 \mathrm{X}}$ and Barone ${ }^{8}$ with the new extended core diffuser highlighted in red. 


\section{Methods}

\section{A. Test Facility}

Tests are conducted in a wind tunnel facility at the University of Virginia ${ }^{8,17}$ (Figs. 3.1 and 3.2). The test section is a full-scale version of the flow path elements of an IPS using similar geometry converted from a two-dimensional axisymmetric geometry. By ensuring full-scale flow path dimensions in a span wise cut and full flow velocities, there was no need to employ Reynolds, Mach, or Stokes number scaling, assuming standard sea-level conditions. To eliminate gravitational bias normal to the flow for the particle trajectories, a vertical pull-down wind tunnel is used. Upstream of the wind tunnel entrance, the particles are dispersed to dilute conditions by compressed air ejection, which prevents undesirable clumping. The resulting particle cloud simulates high particle loading that can occur during austere helicopter landing conditions. The particulate then enters the present wind tunnel facility through an inlet with a flow straightener and a smooth contraction into the test section, where it bifurcates into the scavenge and core legs. Diffusers in each leg (scavenge and core) connect the tunnel to air filters, which collect the test dust to allow separation efficiency measurements. Downstream of the filters, the flow in each leg follows a duct system to its respective blower motor and is then exhausted into ambient (outdoor) conditions. The rig for the present study is the same upgraded version of the previous chapter.

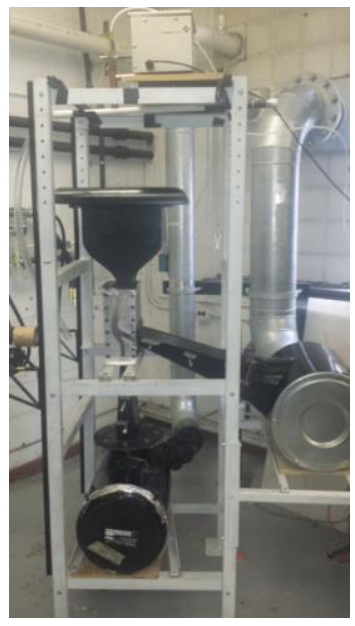

(a)

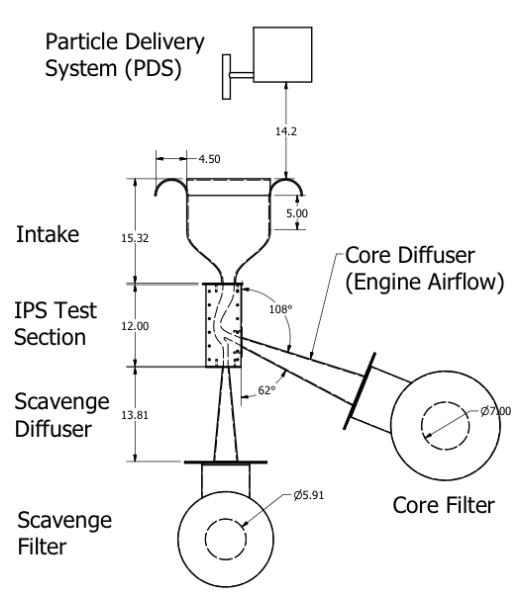

(b)

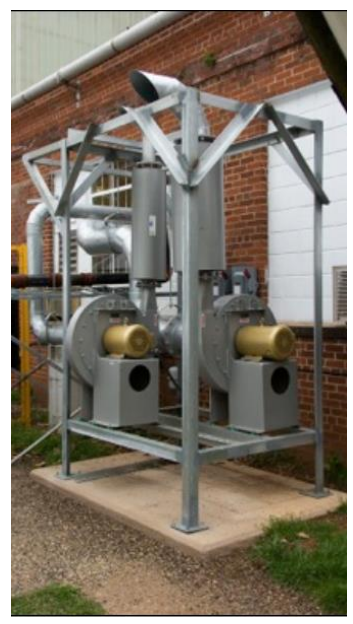

(c)

Figure 3.2. Photographs of the University of Virginia Inertial Particle Separator Facility: (a) vertical tunnel, including inlet, test section, and filter housings where test section of Figure 3.1 is oriented so flow is downward, (b) tunnel schematic (inches), and (c) external blower configuration for both scavenge and core streams. 


\section{B. Mass Flow Control}

The scavenge mass flow fraction $(\beta)$ is defined as the ratio of scavenge leg mass flow rate $\left(\dot{m}_{s}\right)$ compared to the total inlet mass flow rate, which is the sum of the mass flow rates in the core leg $\left(\dot{m}_{c}\right)$ and scavenge leg $\left(\dot{m}_{s}\right)$, i.e.

$$
\beta=\frac{\dot{m}_{S}}{\dot{m}_{S}+\dot{m}_{c}}
$$

The flow is driven by exhaust fans that are controlled to achieve the proper $\beta$ values. During a typical test, the core volumetric flow rate is held ideally constant at 1365 CFM (Cubic Feet per Minute) or $0.6442 \mathrm{~m}^{3} / \mathrm{s}$, a value that is consistent with equivalent flow rates per unit span of an actual engine. Volumetric flow rate is density corrected to the appropriate mass flow rate using the method of the previous chapter. The uncertainty of the scavenge mass flow fraction, $\delta \beta$, is conducted per Barone et al. ${ }^{8}$ :

$$
\delta \beta=\sqrt{\left(\frac{\dot{m}_{s}}{\left(\dot{m}_{s}+\dot{m}_{c}\right)^{2}} \delta \dot{m}_{c}\right)^{2}+\left(\frac{\dot{m}_{s}}{\left(\dot{m}_{s}+\dot{m}_{c}\right)^{2}} \delta \dot{m}_{s}\right)^{2}}
$$

The analysis uses the error in each of the legs, $\delta \dot{m}_{c}$ and $\delta \dot{m}_{s}$, taken as the $2 \%$ reading error reported in the flow meter documented calibration. Note that the uncertainty in the pressure management system is omitted because it is generally two orders of magnitude lower than that of the volumetric flow meters. The values of $\delta \beta$ for all the tests used in this study were about $2 \%$. Full details of the motor controller system may be found in Connolly et al. ${ }^{17}$

\section{Inner Surface Geometry Design and First Order Methods}

As shown in the previous chapter, the hub side of the scavenge channel can play a key role in improving flow stability and particle separation performance. This result was counterintuitive, as the geometry modifications were far downstream of the separation region. Two additional scavenge channel designs were manufactured to examine this effect further. The three Inner Surface Geometries (ISG) under consideration for this study are shown in Figure 3.3a. ISG-1 is the new nomenclature (previously termed medium-hub-downstream) for the best performer in terms of separation efficiency and power consumption from the previous work. ${ }^{17}$ It uses a smooth contraction to promote forward flow in the scavenge channel and prevent downstream instabilities from affecting the test section flow. This idea was taken a step further with ISG-2, by causing the scavenge channel to contract earlier. This aimed to reduce the size of the separation region identified by Barone. ${ }^{11}$ ISG-3 extended even further upstream to further limit the size of the separation bubble. Barone ${ }^{12}$ found that fine particulate tended to be reingested into the core leg after entering 

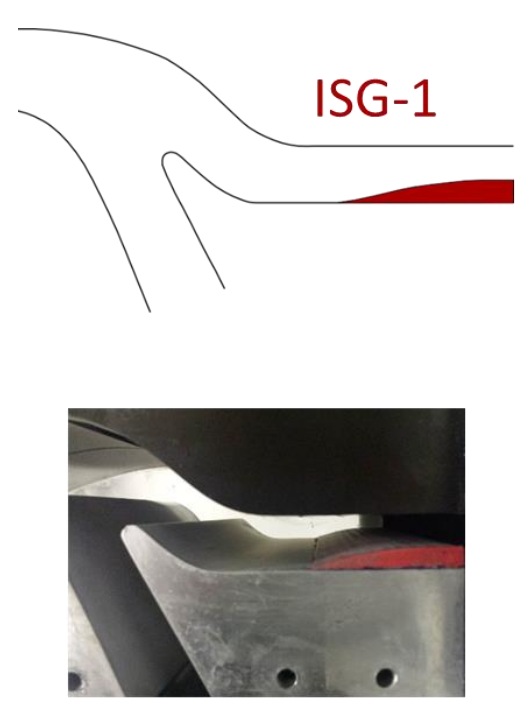

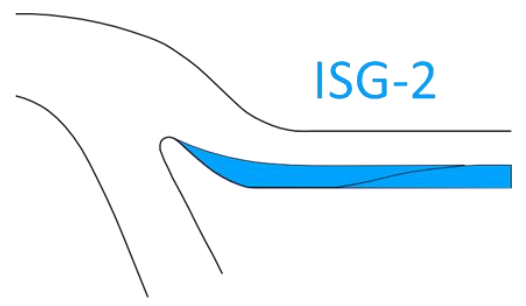

(a)
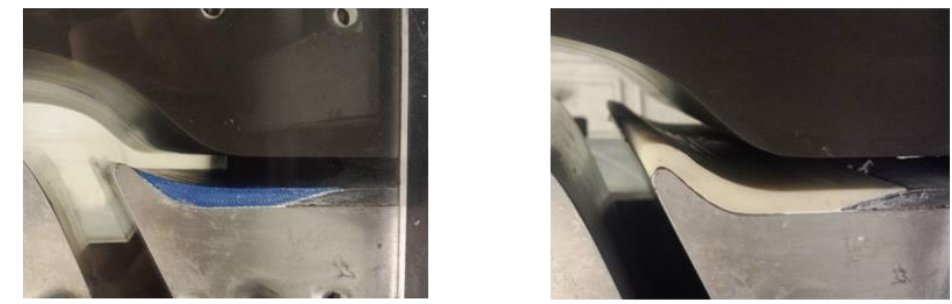

(b)

Figure 3.3. Inner Surface Geometries (ISGs). (a) Schematic of each ISG (b) Each ISG printed out of ABS plastic and affixed in position using silicone sealant. Note that there is a smooth expansion into the scavenge diffuser downstream of the test section.

the scavenge. ISG-3 extends the splitter nose to limit this reingestion while also reflecting larger, bounce-dominated particles into the scavenge.

Due to the possibility of disrupting the behavior of the already well-optimized splitter nose, ISG-3 was considered riskier than the more conservative ISG-1 and ISG-2. Also, since the ISGs are manufactured out of ABS plastic, particle-wall coefficient of restitution is different than that of the aluminum of the rest of the test section. A parametric study of bounce effects will be the subject of future work.

Flow separation occurs when the boundary layer detaches from the wall, in this case due to an adverse pressure gradient. A separation point or line has a wall shear of zero, while the upstream flow direction is forward and the downstream flow direction is reversed. That is, the streamlines upstream and downstream of the separation line point toward it. A thorough overview of flow separation structures can be found in Délery. ${ }^{19}$ Prediction of the location of the initial separation point can be computed to varying degrees of accuracy using computational codes. For a priori estimation of separation for design purposes, it can be more convenient to perform a first order analysis. A method provided by $\mathrm{Cebeci}^{20}$ to estimate separation point from just an inviscid flowfield of the geometry was used in this case to inform ISG design. An inviscid flowfield was calculated using ANSYS Fluent for each OSG. Since the no-slip 
condition cannot be enforced in an inviscid flow, the velocity values along the OSG wall were collected. The coefficient of pressure $\left(C_{p}\right)$ is defined as:

$$
C_{p}=1-\left(\frac{u}{u_{o}}\right)^{2}
$$

where $u$ is the velocity along the wall and $u_{o}$ is the velocity at the onset of the adverse pressure gradient. For this estimation (though not necessarily for the actual flowfield), the adverse pressure gradient is taken to begin at an axial distance of 6 " or $15.24 \mathrm{~cm}$, at the throat. After this point, the channel begins to expand as the flow turns toward the hub side. Cebeci uses Stratford's criteria to predict separation, which is given as:

$$
C_{p}{ }^{1 / 2} x \frac{d C_{p}}{d x}>0.102
$$

This means that separation is predicted when the LHS first reaches a value greater than 0.102 . Note that this method is not intended to make predictions after flow separation occurs. Also, due to the square root term, the method does not make predictions upstream of the onset of separation where $C_{p}$ may be negative. In this case, a first order central difference scheme was used to calculate $d C_{p} / d x$ from the $C_{p}$ values.

\section{Surface Flow Visualization}

Surface flow visualization is used to characterize the large scale flow structures and separation ${ }^{16}$. The tests were conducted with titanium dioxide as the colorant, kerosene as the carrier fluid, and oleic acid as an emulsifier. The mixture was painted on the surface of interest just before operation and the flow then causes the suspension to streak, leaving a time-averaged wall shear pattern as the kerosene slowly dries. Note that the carrier fluid for such

visualization is typically oil and not kerosene, but due to the highly unsteady nature of flow inside the IPS a more volatile fluid was chosen. Kerosene decreases the drying time significantly, allowing the resolution of more transient flow structures often present in separated flows. More details are provided in Connolly et al. ${ }^{17}$ The photographs of the surface flow results have their white balance and contrast adjusted to improve visibility.

\section{E. Separation Efficiency Technique}

To directly measure the particle separation efficiency (by mass) of a given flowpath geometry, approximately $100 \mathrm{~g}$ of test dust is released into the inlet over five minutes of wind tunnel operation by a Particle Delivery System (PDS) volumetric feeder. Once through the test section, the dust is captured in the downstream filters. These filters, as well as the PDS, are weighed before and after each test for determination of the total mass of particulate used in the 
test. The separation efficiency $(\eta)$ is then calculated as the ratio of the mass gained in the scavenge filter $\left(\Delta m_{s f}\right)$ to the total mass gained by both the core filter $\left(\Delta m_{c f}\right)$ and the scavenge filter:

$$
\eta=\frac{\Delta m_{s f}}{\Delta m_{c f}+\Delta m_{s f}}
$$

For example, in the case where $90 \mathrm{~g}$ of the particulate is captured by the scavenge filter and $10 \mathrm{~g}$ by the core filter, 90\% efficiency is achieved. Mass discrepancies $\left(\delta m_{r}\right)$ can occur if particulate sticks to the sidewalls of the tunnel or passes through the filters. These are recorded for each test by comparing the mass change of the PDS ( $\Delta m_{P D S}$, net particles delivered) to the total mass change of the filters $\left(\Delta m_{c f}+\Delta m_{s f}\right.$, net particles collected):

$$
\delta m_{r}=\Delta m_{P D S}-\left(\Delta m_{c f}+\Delta m_{s f}\right)
$$

All efficiency test data in this report have less than $2 \%$ mass discrepancy.

The full procedure for measuring particulate separation efficiency is detailed in full Connolly et al. ${ }^{17}$, with the following minor additions. Preventing clumping of dispersed particulate is of special concern when trying to accurately measure separation efficiency. This is because a group of clumped particles will have very different aerodynamic characteristics compared to the same particles when dispersed. In response, all particulate used in this study was unsealed from its container and heated in an oven to between $55 \mathrm{C}$ and $65 \mathrm{C}$ for at least thirty minutes before testing. This aimed to reduce any moisture content that may have been present. Additionally, the PDS was cleaned out thoroughly before every test to ensure consistent operating conditions. Previous work ${ }^{10,17}$ have not shown any visible signs of particulate clumping, but the potential for high atmospheric humidity and the sensitivities of working with finer particulate in the future prompted these prudent additions to the test procedure.

\section{F. A4 and AFRL 03 Test Particulate}

Research by Barone et al. ${ }^{10}$ focused on A4 test particulate. With a peak volumetric Probability Density Function (PDF) diameter of approximately $35 \mu \mathrm{m}$, A4 contains smaller drag-dominated particles $(\mathrm{St}<<1)$, larger bouncedominated particles (St $>1$ ), and those in between the two regimes (St 1). AFRL 03, by contrast, is made up of finer particulate with a peak diameter around $20 \mu \mathrm{m}$, so it consists primarily of intermediate or drag-dominated particles (Fig. 3.4). Particle size distributions vary slightly (well within manufacturer specifications) from batch to batch, with data for some of the particulate used in this study in Table 3.1. Lower Stokes number particles are more dependent on flowfield stability for separation efficiency, and sub-micron particulate is especially difficult to filter, which makes them an excellent candidate to test on the IPS. Additional details on A4 can be found in Barone et al. ${ }^{10}$ 
AFRL 03 consists of a mixture of 34\% quartz, 30\% gypsum, 17\% aplite, 14\% dolomite and 5\% salt

(http://www.powdertechnologyinc.com). Qualitatively, A4 appears like a very fine brown beach sand, while AFRL

03 more resembles talcum powder.

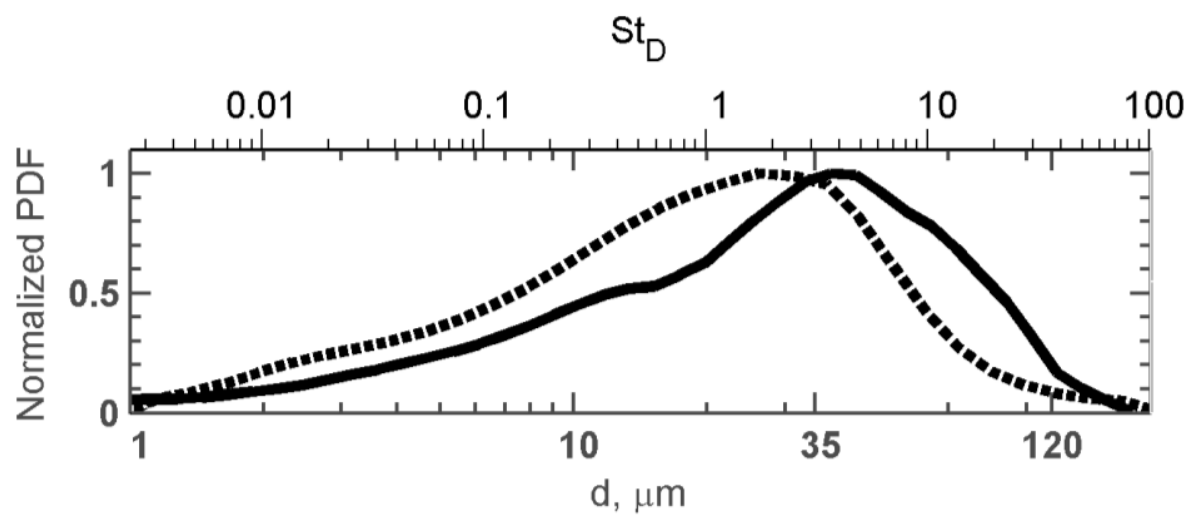

...".." AFRL $03 \longrightarrow$ A4

Figure 3.4. Test Particulate Normalized Probability Functions (PDF). The particle diameters and their associated Stokes number are plotted against their normalized probability. (Data provided by supplier, Powder Technology Inc.)

\begin{tabular}{c|c|c} 
bin $\%$ & A4 diameter $(\mu \mathrm{m})$ & AFRL 03 diameter $(\mu \mathrm{m})$ \\
\hline 10 & 4.44 & 3.67 \\
20 & 10.06 & 6.71 \\
30 & 16.86 & 10.01 \\
40 & 24.64 & 13.59 \\
50 & 33.74 & 17.71 \\
60 & 44.12 & 22.59 \\
70 & 56.75 & 28.53 \\
80 & 73.81 & 36.30 \\
90 & 105.1 & 49.34 \\
95 & 140.9 & 64.41
\end{tabular}

Table 3.1. Test Particulate Distributions. Each bin gives the percentage of particulate (by volume) that is smaller than the given diameter. For example, for A4, 50\% of the particulate by volume has a smaller diameter than 33.74 $\mu \mathrm{m}$. (Data provided by supplier, Powder Technology Inc.) 


\section{G. PIV Technique}

Particle Image Velocimetry (PIV) is a method commonly used in gas flows to quantify the velocity field. It employs tracer particles seeded into the flow that are small enough in size so that they faithfully follow the surrounding airflow. The experimental approach used herein utilizes olive oil droplets of approximately $1 \mu \mathrm{m}$ diameter, because they very closely follow the flow due to their low Stokes number. These particles are illuminated with a laser sheet, and tracked using a camera. The flow's velocity field is calculated using a pair of captured images that contain the same set of particles after they have travelled a short distance. The field is subdivided into a discrete grid of subregions referred to as interrogation spots to obtain a two-dimensional matrix of velocity vectors. For each of these sub-regions, the particles that have traversed a small distance across two successive images are cross-correlated. The distance vector from the cross-correlation is divided by the time between the two images resulting in the velocity vector. By taking a large number of these image pairs throughout an experiment, an average flow field can be determined, along with the statistical variations present.

In the present set-up, the laser sheet is positioned at the centerline of the test section width so as to provide the clearest view of the flow at the bifurcation in order to characterize the flow in the region associated with the highest amounts of instability. The PIV camera is used to capture approximately 1000 image pairs, which are interrogated to examine instantaneous flow structures and velocity vectors. The camera records image pairs at a rate of $7 \mathrm{~Hz}$, which is sufficient for capturing instantaneous flow velocities and structures. This data was averaged to obtain the mean velocity and standard deviation of the velocity of the flow. This allows gas velocity to be measured within $2 \%$ accuracy. ${ }^{11}$ Atmospheric conditions were recorded before each experiment (Table 3.2). Scavenge mass flow fraction was set to $\beta=0.10$ for each geometry, since the flowfield is more sensitive to separation at lower scavenge mass flow rates. Further details of the PIV equipment and methods are described by Barone et al. ${ }^{11-12}$ In addition, the view window of the current study extends further into the scavenge leg to better investigate flow separation and unsteadiness in that region.

\begin{tabular}{ccccccc} 
ISG & Seed & $\beta$ & $\mathrm{T}$ & $R H$ & $p$ & $\mathrm{~N}$ \\
\hline 1 & Olive Oil & 0.10 & $74.1 \mathrm{~F}$ & $48 \%$ & $29.2 \mathrm{inHg}$ & 1000 \\
2 & Olive Oil & 0.10 & $72.0 \mathrm{~F}$ & $35 \%$ & $29.0 \mathrm{inHg}$ & 1000 \\
3 & Olive Oil & 0.10 & $75.8 \mathrm{~F}$ & $54 \%$ & $29.5 \mathrm{inHg}$ & 1000
\end{tabular}

Table 3.2. Operating conditions at time of each PIV capture 


\section{Results}

Investigations of novel Inner Surface Geometries (ISGs) were performed on a full-scale Inertial Particle Separator test rig. Surface flow visualization, A4 particle separation efficiency, and PIV experiments were conducted for each of the three ISGs. Additionally, AFRL 03 particle separation efficiency was evaluated on ISG-1.

\section{A. Surface Flow Visualization Results}

The results of the surface flow visualization for each ISG are shown in Figure 3.5a, with a flowfield interpretation in Figure 3.5b. Of particular interest are the irregular separation lines, similar to those observed in Trünkle et al. ${ }^{16}$ The separation lines are not straight as might be expected for a quasi-2D flow. The sidewall separation is largely contained for each case. The flow reattachment line is poorly defined, indicating a variably sized separation region. Downstream of the bifurcation, flowlines reappear, suggesting attached flow exiting the scavenge. All three ISGs have approximately similar separation points. Due to their distance downstream, it was not likely that the ISGs could affect the onset of separation, only the separation region and reattachment. Note that ISG-3 has the thinnest separation region, perhaps indicating that it is promoting flow reattachment successfully.

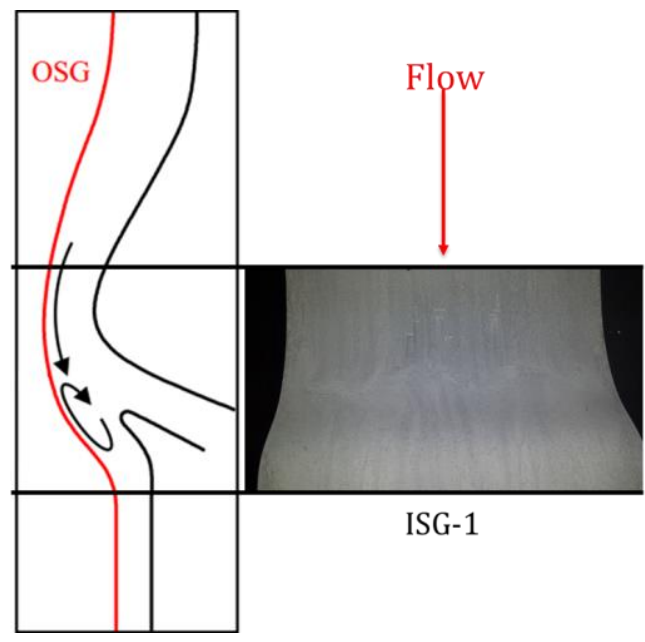

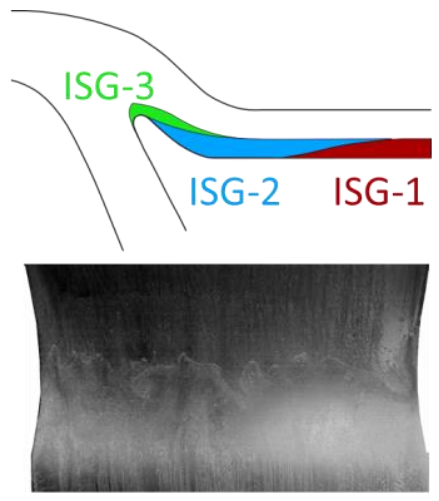

ISG-2

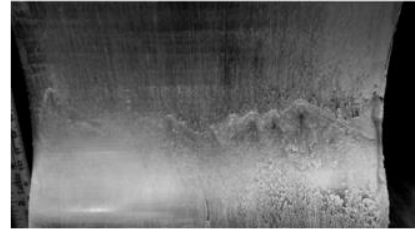

ISG-3

Figure 3.5a. Surface Flow Visualization

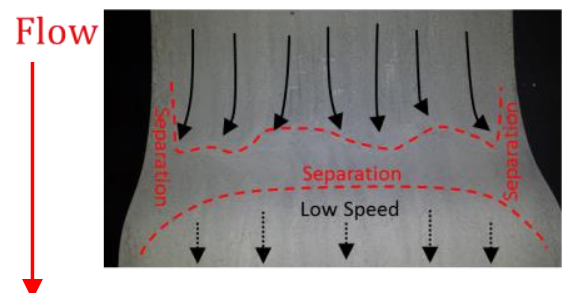

ISG-1

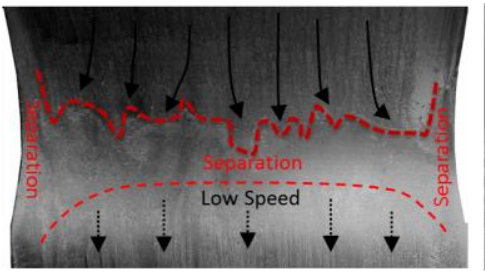

ISG-2

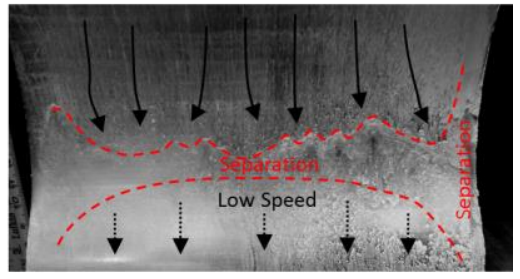

Figure 3.5b. Surface Flow Visualization Interpretation 


\section{B. Stratford's Criteria and PIV Results}

The results of the simple inviscid flowfield (Fig. 3.6) show that decreasing the width of the scavenge channel results in lower pressures and higher velocities, as expected. The simulations are not meant to be representative of the actual flowfield inside the IPS, but provide the necessary data to predict flow separation using Stratford's criteria. The coefficient of pressure $\left(\mathrm{C}_{\mathrm{p}}\right)$ and its gradient are computed along the OSG wall from the inviscid solutions (Fig. 3.7a). The estimated location of the onset of the adverse pressure gradient is 6 " $(15.24 \mathrm{~cm})$ in the axial direction. The area of interest for separation is marked with vertical red lines. The result of the calculation of Stratford's Criteria for each ISG is shown in Figure $3.7 \mathrm{~b}$ (zoomed to the area of interest), along with the experimental data of the time averaged PIV plots. The predicted separation point is marked with a red $\mathrm{X}$ at the same axial location on each plot. Note that each ISG is predicted to separate at the same point, due to identical upstream geometries. Only ISG-1 has fully developed separation appear in the mean results, but strong mean velocity gradients in the PIV of all ISGs suggest that Stratford's criteria is successful in approximating the onset of separation.

ISG-1

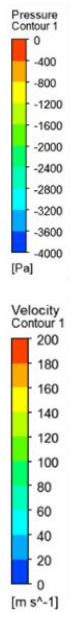

ISG-2
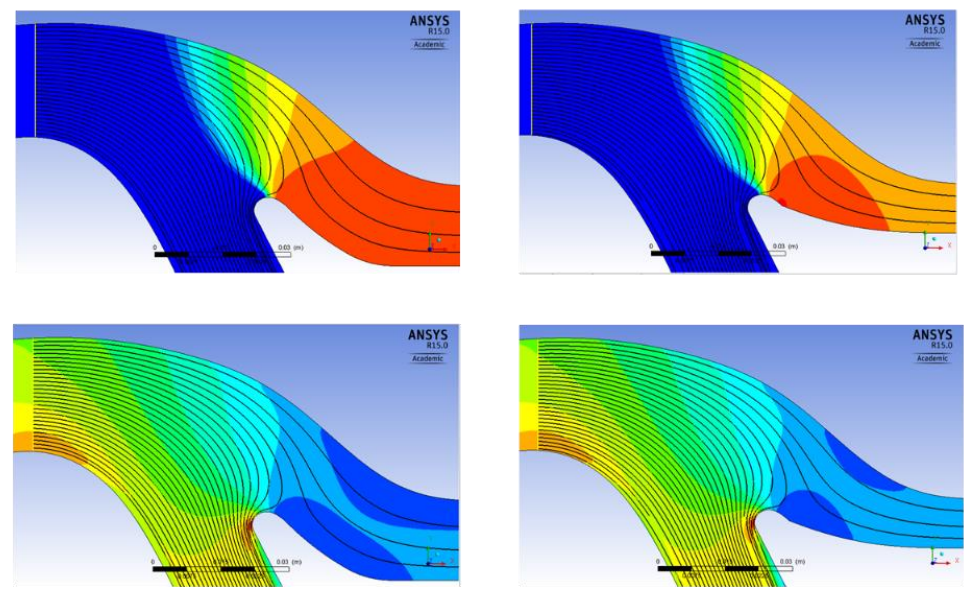

ISG-3
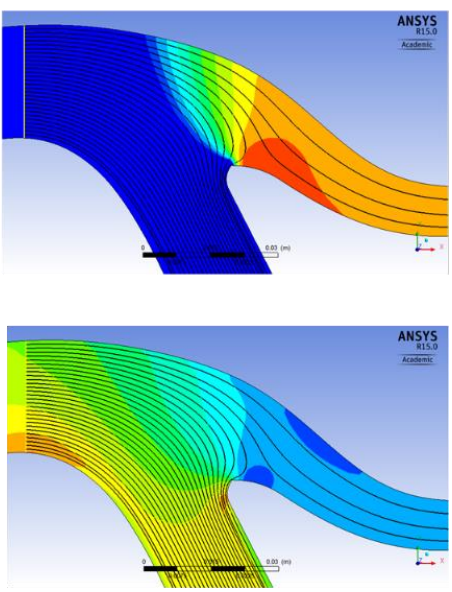

Figure 3.6. 2D Inviscid CFD Pressure and Velocity Contours at $\beta=10 \%$. 


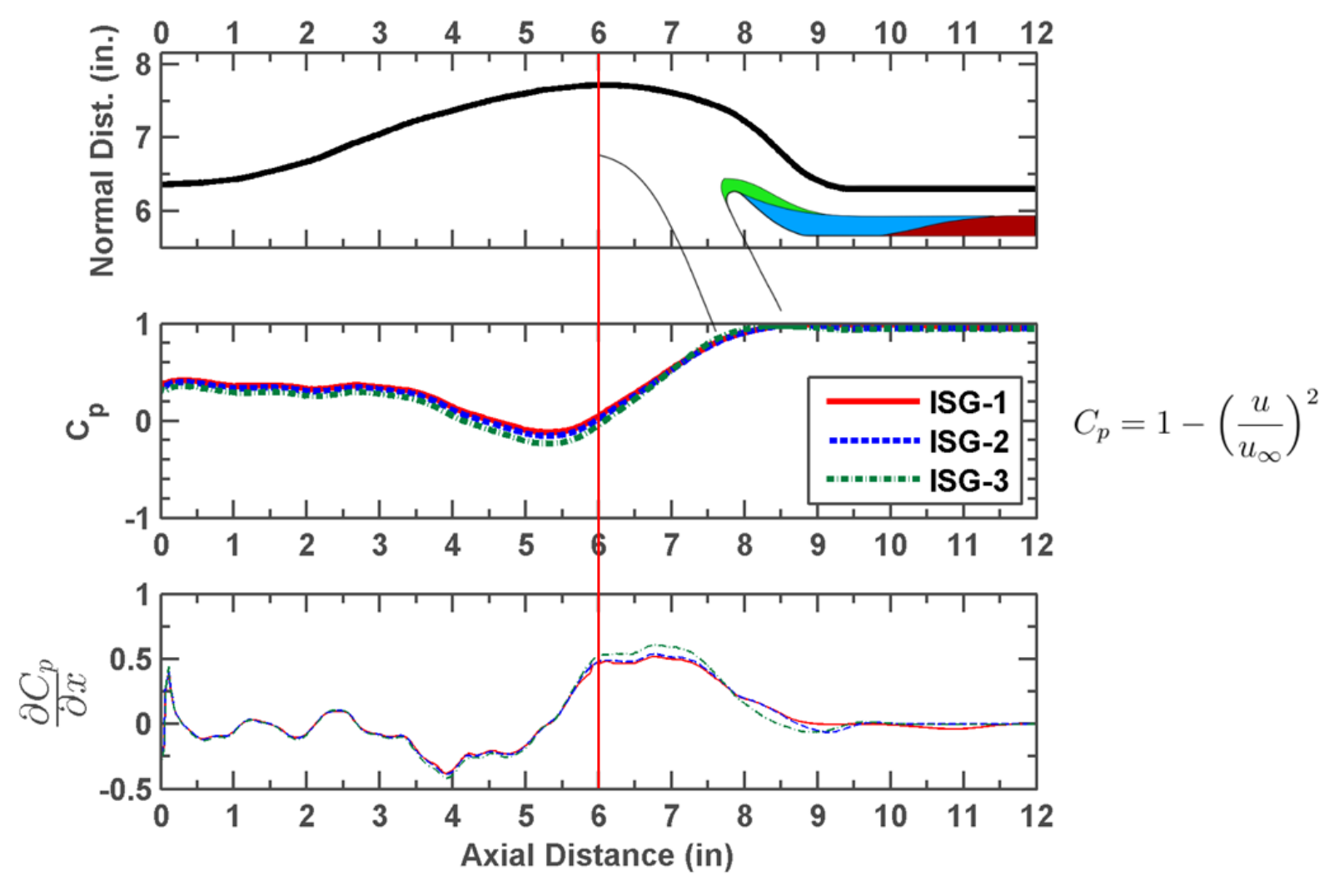

Figure 3.7a. Statistics from Inviscid Flow Solution at $\beta=0.10$
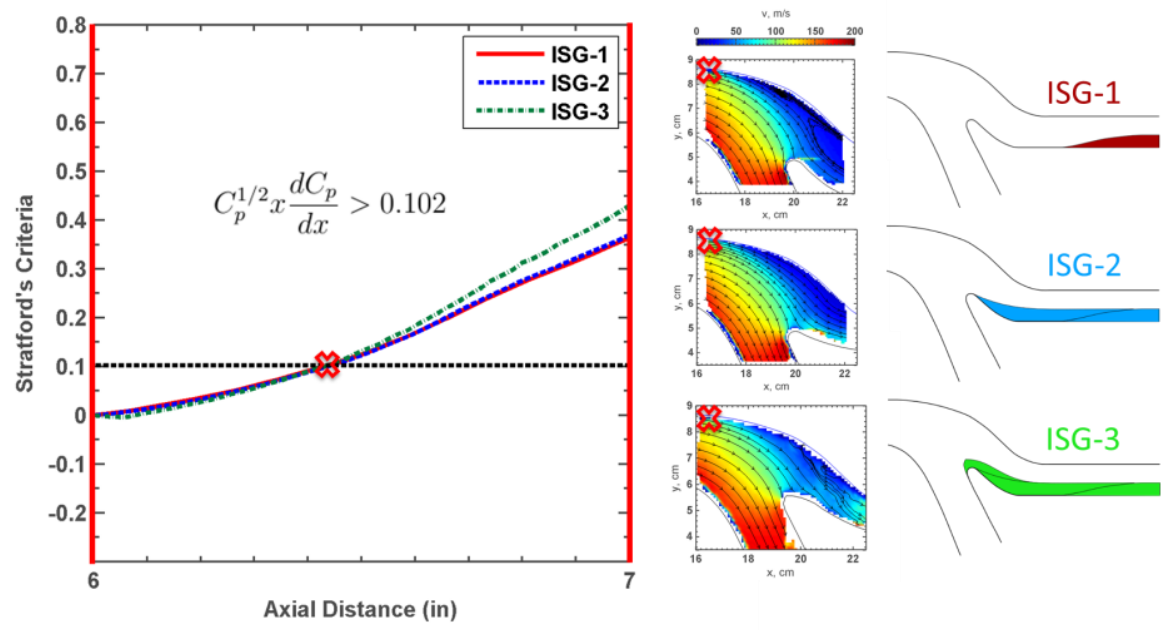

Figure 3.7b. Stratford's Criteria of Separation Prediction with Time Averaged PIV Results at $\beta=0.10$. Stratford's criteria predicts nearly identical separation for each ISG. Time averaged PIV results (right) suggest that this prediction is accurate for the mean flowfield 
The time averaged PIV statistics at the relatively low scavenge flow ratio of $\beta=0.10$ are presented in Figure 3.8. The velocity fields (Fig. 3.8a) for each ISG are similar at the throat and core flow regions, which is to be expected since geometry modifications were confined to the scavenge. ISG-1 shows a large mean recirculating region along the OSG wall, nearly blocking the scavenge inlet. The PIV view window for this study extends further into the scavenge leg, which explains why this large recirculation region was not observed at the same flow conditions in Connolly et $a l .{ }^{17}$ The mean recirculation seen here begins just downstream of the view window of the previous study. ISG-2 and ISG-3 show mean forward flow throughout the scavenge channel, and ISG-3 shows higher velocities as well. The reduced scavenge channel height may have an effect of suppressing flow separation, promoting reattachment, or both. Flow standard deviation (Fig. 3.8b) shows what appears to be increased unsteadiness for ISG-2, and especially ISG3. Note that laser reflections on the splitter nose cause some measurement noise in this region for all ISGs. Additionally, the confined measurement space and seed particle accumulation on the viewing window may account for some of the high standard deviation for ISG-3. Standard deviation normalized by local mean velocity (Fig. 3.8c) suggests a high amount of unsteadiness even in ISG-1. This is indicative of highly transient separated flow in all three flowfields.
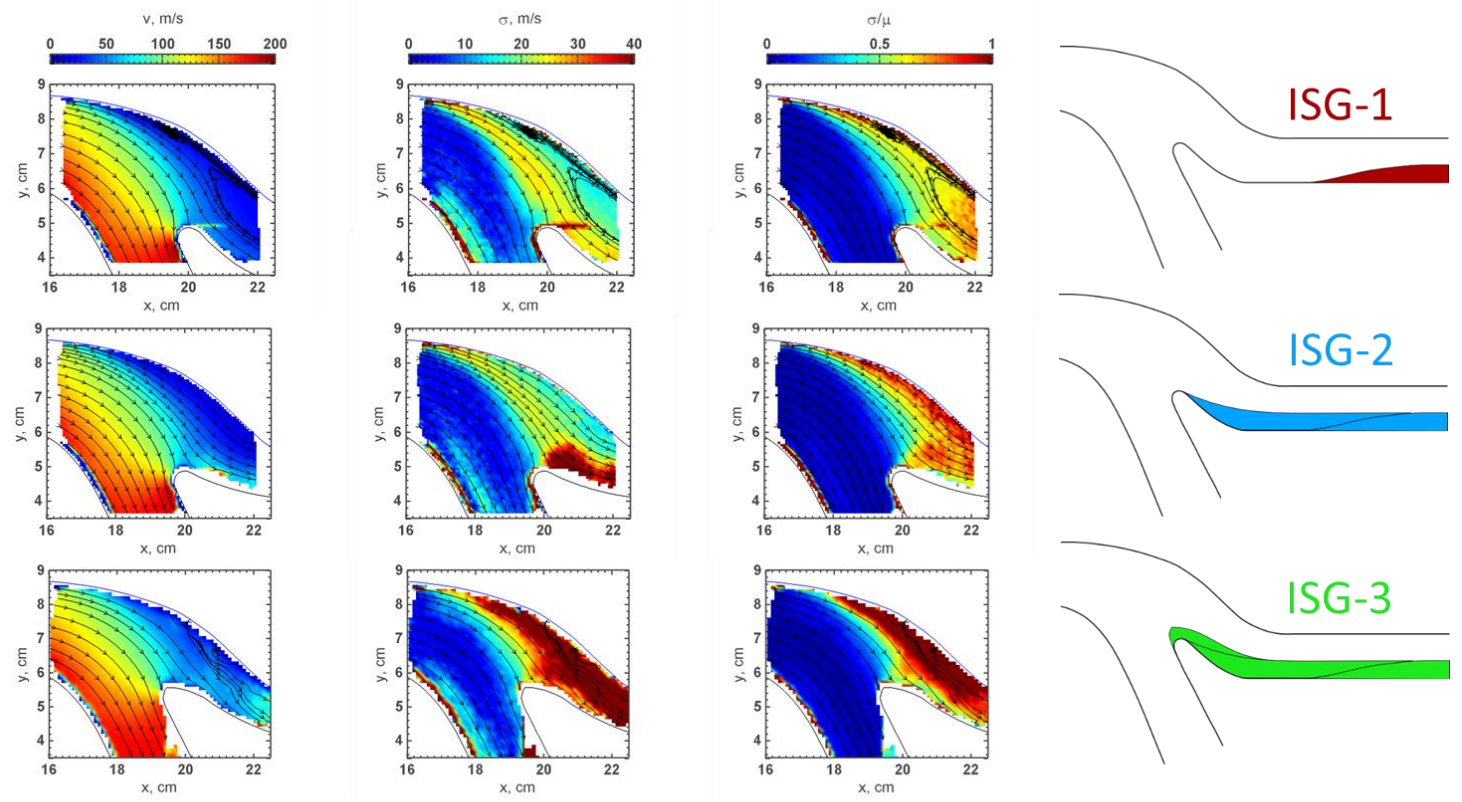

(a)

(b)

(c)

Figure 3.8. Time Averaged PIV Statistics at $\boldsymbol{\beta}=\mathbf{0 . 1 0}$. (a) Velocity contours show similar mean flowfields in the core region, while a large mean recirculation region is visible for ISG-1. ISG-2 and ISG-3 do not show mean reversal. (b) Standard deviation is much higher for ISG-3. (c) Standard deviation normalized by the local mean velocity shows that the most significant flow fluctuations occur in the scavenge leg. 
Instantaneous snapshots of flow (Fig. 3.9) can vary wildly from the mean flow. Significantly, this variation is observed for each ISG. The flow separation can completely block the scavenge channel (Fig 3.8a), which may push smaller particles into the core stream. "Typical" IPS operation may appear similar to Figure 3.8b, where separation is limited to the vicinity of the OSG, and a steady stream of flow is entering the scavenge. This also most resembles the mean flowfield. Large reingestion events (Fig. 3.8c) not only block the entire scavenge, but significantly disrupt the core flowfield. This means that not only is all small particulate $(\mathrm{St}<1)$ being reingested, but disruption of the core flow could be adding significantly to pressure losses. This type of event was filmed on high speed video by Barone et al. ${ }^{12}$ and termed a wall normal vortex, due to the apparently vortical nature of the reingestion.
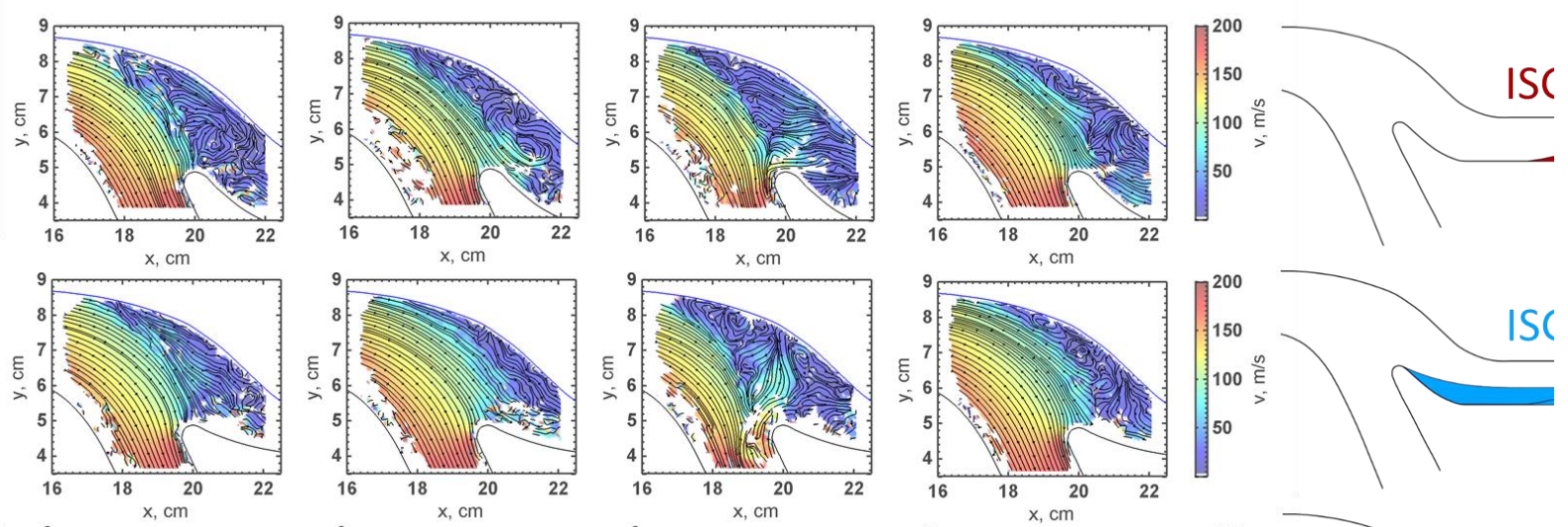

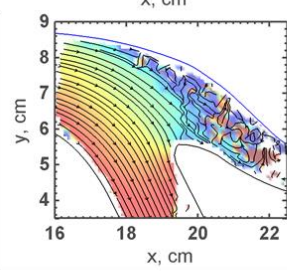

(a)

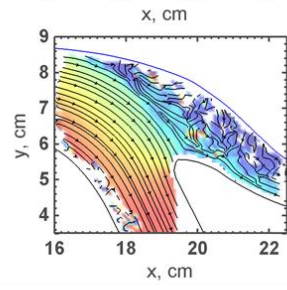

(b)

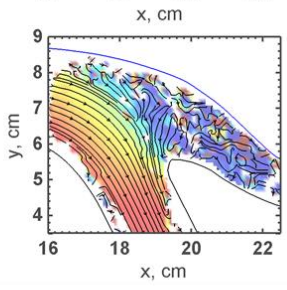

(c)

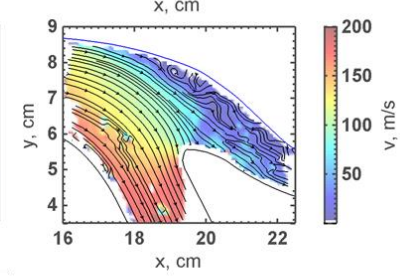

(d)

Figure 3.9. Instantaneous PIV Samples at $\boldsymbol{\beta}=\mathbf{0 . 1 0}$. Highly transient flow with wide variation in size of recirculation zone. For any ISG, flow can be characterized as (a) separated and blocking the scavenge channel, (b) mildly separated with forward scavenge flow (similar to the mean), (c) forced into core reingestion by a wall normal vortex, or (d) have forward scavenge flow with downstream separation.

Large reingestion vortices appear in all three ISGs, regardless of geometry. Rather than preventing flow separation as suggested by the mean flows, ISG-2 and ISG-3 may simply be reducing its incidence. The 3D nature of the separation $^{12}$ suggests that flow most likely varies significantly along the span. These sample images do not represent a comprehensive view of all the observed separation flow structures, but demonstrate the variety of flow conditions 
observed during regular IPS operation. Note that the narrow channel of ISG-3 makes data collection in the scavenge channel somewhat difficult, hence the poorer image quality.

A control volume for the flow bifurcation region (Fig. 3.10) shows mean flow profiles for the throat, core, and scavenge from the PIV data. These velocity rakes allow more quantitative comparison of the PIV data than the contour plots. Note that the central figure's geometry is ISG-3 for illustrative purposes only (ISG-2, by comparison, is shown at the bottom right). The throat velocity profiles (Fig. 3.9a) are primarily uniform and consistent between ISGs. The core profiles (Fig. 3.9b) are nearly identical for ISG-1 and ISG-2, but ISG-3 has a moderately higher velocity. This can be explained by two factors. First, the geometry of ISG-3 extends slightly into the core flow and may be slightly altering the flowfield. Second, ISG-3 blocks a portion of the PIV laser's path into the core, as denoted by the shadow region in the figure, preventing data collection near the wall. The scavenge profiles show higher velocities for ISG-2 and ISG-3. Since ISG-3 has a smaller scavenge channel height, its rake does not extend as far in the y direction that ISG-1 or ISG-2. Note also that the mean velocity of ISG-1 is negative near the OSG wall, indicating the beginning of the recirculatory flow seen in the mean contour plots.

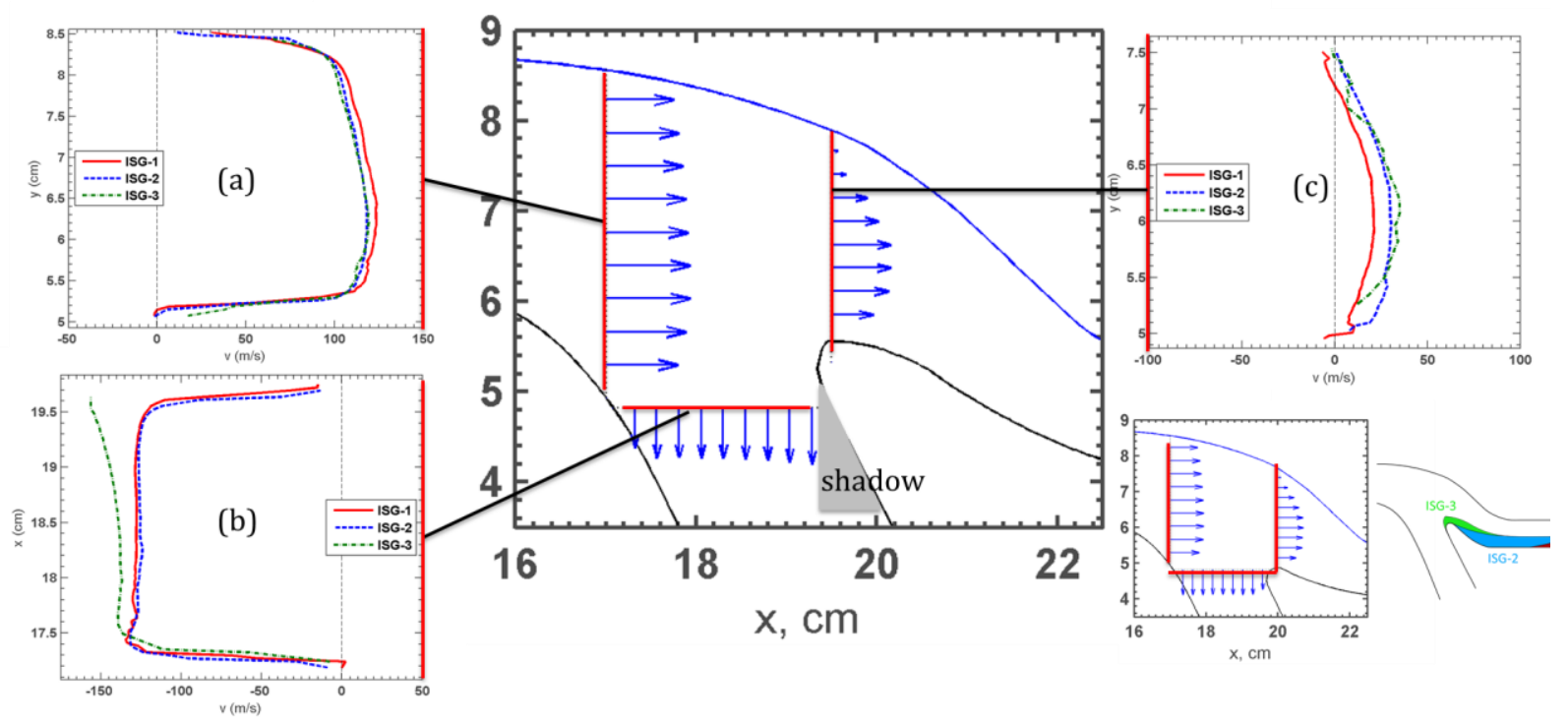

Figure 3.10. Control Volume Velocity Rakes at $\beta=0.10$. (a) Throat velocity profiles (b) Core velocity profiles (c) Scavenge velocity profiles 


\section{Separation Efficiency Results}

A4 separation efficiency results for all ISGSs are shown in Figure 3.11a. The majority of efficiency tests were performed on the baseline case, ISG-1. At $\beta=0.16$, separation efficiency $(\eta)$ is just above $90 \%$ for all cases, which is in line with previous studies. ${ }^{10,17}$ ISG-2 and ISG-3 are slightly below ISG-1 at this condition, but only by 1-3\%. The nearly equivalent separation efficiency is consistent with the similar oil streak results at $\beta=0.16$. Above $\beta=0.12, \eta$ for A4 is primarily linear with respect to $\beta$. However, below that point, flow separation is theorized to cause a severe drop-off in $\eta .{ }^{17}$ The ISG-1 results align with that interpretation. However, note that ISG-2 and ISG-3 do not experience as severe a drop-off. Both have slightly higher efficiency than ISG-1 at $\beta=0.10$ and far outperform ISG-1 at $\beta=0.09$, by $8-9 \%$. Recall that the PIV data at $\beta=0.10$ showed a significant mean recirculation zone for ISG-1, while ISG-2 and ISG-3 had mean forward flow in the scavenge. This is a major improvement in separation efficiency for relatively minor geometry changes.

AFRL 03 separation efficiency on ISG-1 (Fig. 3.11b) is significantly lower than for A4, as expected. AFRL 03 separation efficiency results are approximately $10 \%$ below A4 for the range of investigated $\beta$. This data provides an important baseline for evaluating future modifications to the IPS with respect to particle size.

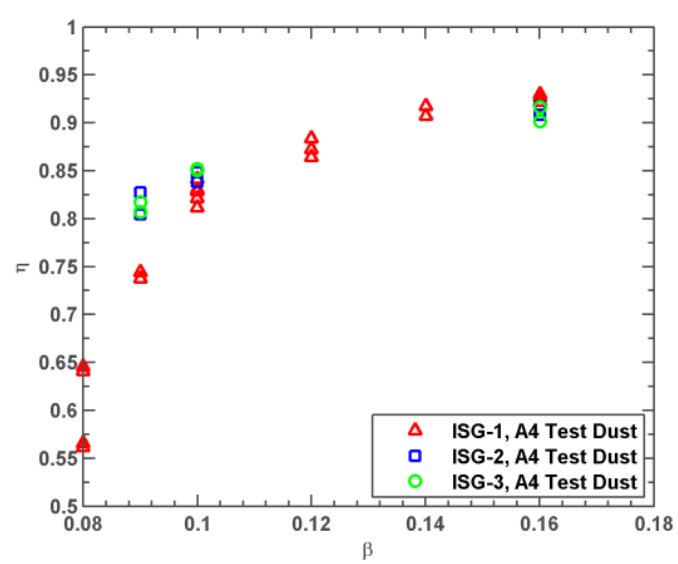

(a)

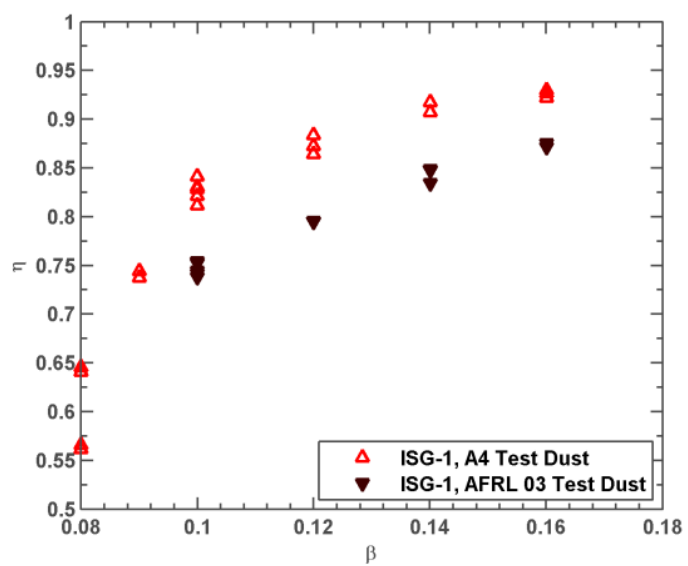

(b)

Figure 3.11. Particle Separation Efficiencies. (a) A4 test dust separation efficiency for ISG-1, ISG-2, and ISG3. Note the superior performance of ISG-2 and ISG-3 at low $\beta$. (b) AFRL 03 test dust separation efficiency for ISG-1 only. 


\section{Conclusions}

Investigations on scavenge flowpath geometry and particle size were conducted at a fundamental two-dimensional Inertial Particle Separator facility. New Inner Surface Geometries (ISGs) were designed to reduce flow separation in the scavenge leg, with the aim of improving particle separation efficiency. These were evaluated at a wide range of scavenge mass flow fractions $(\beta)$. Surface flow visualization showed similar flow separation for each geometry at the relatively high $\beta$ of 0.16 , where flow unsteadiness is less of a concern. A first-order method given by Cebeci and based on the inviscid flowfield was successfully used to predict the location of the onset of flow separation when compared to surface flow visualization. It may be employed to quickly prototype new geometries with negligible computational cost.

The mean PIV flowfield of the baseline ISG- 1 at $\beta=0.10$ revealed a significant mean recirculation region in the scavenge channel. The flowfields of ISG-2 and ISG-3 did not have a visible mean recirculation region, and their mean scavenge velocities were higher than ISG-1. Instantaneous PIV confirmed that flow separation and core reingestion occurs with all three geometries. However, the differences in the mean flowfields suggest that ISG-2 and ISG-3, by decreasing the scavenge channel height, reduce the incidence rate of flow separation and reversal. Mean core flow was faster with ISG-3, most likely due to its modifications of the splitter nose.

ISG-2 and ISG-3 outperformed ISG-1 in terms of particle separation efficiency $(\eta)$ of A4 test particulate at low $\beta$. This is a significant result, since lower $\beta$ is associated with lower system power costs. These ISG modifications reduced scavenge channel height by moving the inner surface up, toward the Outer Surface Geometry (OSG). These values for $\eta$ at low $\beta$ are very similar to those obtained in the previous chapter. That study decreased the scavenge channel height by moving the OSG down, toward the inner surface. The original geometry (Fig. 3.1a) is finely tuned to catch large, bounce-dominated particles $(\mathrm{St}>1)$. The risk with the types of modifications in these studies is that they will interfere with the bounce profiles, causing large particles to end up in the core. Whether that is an issue remains to be seen, but the new geometries are on par or have better performance than the original geometry with A4. This suggests the benefits of removing significantly more of the smaller particles may outweigh the risk of adversely affecting bounces.

AFRL 03 baseline separation efficiency data was also collected for ISG-1. This provides an important reference case for future investigations into particle size effects, which are of great importance to IPS development and improvement. 


\section{Conclusions}

\section{General Conclusions}

The study objectives outlined in the introduction were successfully accomplished. Novel geometries were evaluated based on a variety of performance criteria, and a number of improvements over existing designs were found. The primary hypothesis linking reductions in flow instability with increases in particle separation efficiency was confirmed for a variety of geometries.

Channel contractions (Ch. 1) especially proved effective for the power efficient condition of low scavenge mass flow fraction $\beta$. The best designs limited flowfield instability without compromising the large particle bounce profiles, as designed. This reduction of flow instability had the added benefit of further reducing system power costs.

A novel, more restricting Outer Surface Geometry (OSG) outperformed similar designs by Barone (Ch. 2) The scavenge channel to throat height ratio s/H was found to be an important parameter for designing the OSG. Reductions in $\mathrm{s} / \mathrm{H}$ for a new geometry were correlated with improvements in particle separation efficiency, particularly at low $\beta$. Flowfield results showed that decreasing s/H was strongly related to a decrease in flow instability. For future work, care must be taken not to reduce $\mathrm{s} / \mathrm{H}$ to the point where large particle bounces are adversely affected.

Modifications to the upstream scavenge channel and splitter nose (Ch. 3) also showed improvement to particle separation efficiencies at low $\beta$. These more extreme scavenge channel contractions outperformed their predecessors of Chapter 1, and appeared especially good at reducing flowfield instabilities at low $\beta$.

\section{Recommendations}

Additional test methodology could benefit understanding of this area. Particle PIV and High Speed PIV are both experimental methods that would elucidate some of the more complex particle and flowfield dynamics that occur in an operational IPS. In this vein, particle separation efficiency tests on smaller particulate, such as AFRL 03, could provide insight into a more specific Stokes number dependence on separation efficiency and flowfield. Additionally, testing on larger particulate is required to ensure bounce profiles are not adversely affected by new designs.

Future design work on this type of IPS design could benefit from incorporating the features of a number of these designs. Specifically, new OSGs and ISGs could be shaped even more aggressively to further reduce flow instability. 
Proper understanding of the highly complex flow physics is critical to advancing the state of the art in this field. Critically, proper and extensive experimental data is critical for developing and validating numerical models.

This research suggests that ongoing improvements to operational IPS systems is highly possible and desirable. Reductions in engine damage as a result of particulate ingestion are achievable through geometry modification. 


\section{References}

${ }^{1}$ Stallard, P., "Helicopter Engine Protection,” Perfusion, Vol. 12, No. 4, 1997, pp. 263-267.

${ }^{2}$ Fillipone, A., \& Bojdo, N., "Turboshaft Engine Air Particle Separation,” Progress in Aerospace Sciences, Vol. 46, 2010, pp. 224-245.

${ }^{3}$ Saeed, F., \& Al-Garni, A., “Analysis method for inertial particle separator," Journal of Aircraft, Vol. 44, 2007, pp. $1150-1158$.

${ }^{4}$ Hamed, A., Tabakoff, W., \& Wenglarz, R., "Erosion and deposition in turbomachinery," Journal of Propulsion and Power, Vol. 22, 2006, pp. 350-360.

${ }^{5}$ Fillipone, A., \& Bojdo, N., "Fabric Filter Performance of Helicopter Inlet Barrier Filters," Journal of the American Helicopter Society, Vol. 57,(3), 2012, pp. 1-13.

${ }^{6}$ Bentley, E. R., Sisson, L., "Joint Future Vertical Lift Initiative," Proceedings of the 71 st Annual Forum of the American Helicopter Society, Virginia Beach, VA, 2015.

${ }^{7}$ Abedi, M., "Effect of Restitution Coefficient on Inertial Particle Separator's Efficiency," Master's Thesis, Department of Mechanical and Industrial Engineering, Northeastern University, Boston, MA, 2009.

${ }^{8}$ Barone, D., Loth, E., Snyder, P., “A 2-D Inertial Particle Separator Research Facility,” 28th AIAA Aerodynamic Measurement Technology, Ground Testing, and Flight Testing Conference, New Orleans, LA, 2012.

${ }^{9}$ Snyder, P., "Particle separator for a gas turbine engine," US Patent 6,698,180, March 2, 2004.

${ }^{10}$ Barone, D., Hawkins, J., Loth, E. Snyder, P., "Efficiency of an Inertial Particle Separator", 51st AIAA Aerospace Sciences Meeting, Grapevine, TX, 2013.

${ }^{11}$ Barone, D., Loth, E., Snyder, P., "Fluid Dynamics of an Inertial Particle Separator," 52nd AIAA Aerospace Sciences Meeting, National Harbor, MD, 2014.

${ }^{12}$ Barone, D., "Inertial Particle Separator Multiphase Dynamics," Ph.D. thesis, University of Virginia, 2014.

${ }^{13}$ Jiang, L. Y., Benner, M., Bird, J., “Assessment of Scavenge Efficiency for a Helicopter Particle Separation System,” Journal of the American Helicopter Society, Vol. 57, (2), Apr. 2012, pp. 1-8.

${ }^{14}$ Giacomo, P., "Equation for the determination of the density of moist air," Metrologia, Vol. 18, pp. 33-40. 
${ }^{15}$ Mattingly, J., Elements of Propulsion: Gas Turbines and Rockets, AIAA, New York, NY, 2006, pp. 446.

${ }^{16}$ Trünkle, J., Abdelouahab, M., Wiess, M., "Fluctuating Pressure Measurements in a Turbulent Separation Bubble." Comptes Rendus Mecanique, Vol. 344, 2016, p. 60-67.

${ }^{17}$ Connolly, B., Loth, E., Smith, E.L., Snyder P., "Influence of Scavenge Geometry on Inertial Particle Separator Performance," 54th AIAA Aerospace Sciences Meeting, San Diego, CA, 2016

${ }^{18}$ Runstadler, P.W., Dolan, F.X. and Dean, R.C., Diffuser Data Book, TN-186, Creare, Inc. Hanover, New Hampshire, 1975.

${ }^{19}$ Délery, J. Three Dimensional Separated Flow Topology: Singular Points, Separation Lines and Vortical Structures, Wiley, London, UK, 2013.

${ }^{20}$ Cebeci, T. Analysis of Turbulent Flows, Elsevier, London, 2004, p.242. 